Article

\title{
Comprehensive Optical Investigations of Charge Order in Organic Chain Compounds (TMTTF) ${ }_{2} \mathrm{X}$
}

\author{
Martin Dressel ${ }^{1} *$, Michael Dumm ${ }^{1}$, Tobias Knoblauch ${ }^{1}$ and Matteo Masino ${ }^{2}$ \\ ${ }^{1}$ Physikalisches Institut, Universität Stuttgart, Pfaffenwaldring 57, D-70550 Stuttgart, Germany; \\ E-Mails:dumm@pi1.physik.uni-stuttgart.de (M.D.); \\ tobias.knoblauch@pi1.physik.uni-stuttgart.de (T.K.) \\ ${ }^{2}$ Dip. Chimica Generale ed Inorganica, Chimica Analitica e Chimica Fisica, Università di Parma, Parco \\ Area delle Scienze, 43124-I Parma, Italy; E-Mail: matteo.masino@unipr.it
}

*Author to whom correspondence should be addressed; E-Mail: dressel@pi1.physik.uni-stuttgart.de; Tel.: +49-711-6856-4946; Fax: +49-711-6856-4886.

Received: 2 March 2012; in revised form: 30 April 2012 / Accepted: 1 May 2012 /

Published: 23 May 2012

\begin{abstract}
Charge ordering in the (TMTTF $)_{2} X$ salts with centrosymmetric anions $\left(X=\mathrm{PF}_{6}^{-}\right.$, $\mathrm{AsF}_{6}^{-}, \mathrm{SbF}_{6}^{-}$) leads to a ferroelectric state around $100 \mathrm{~K}$. For the first time and in great completeness, the intra- and intermolecular vibrational modes of (TMTTF) ${ }_{2} X$ have been investigated by infrared and Raman spectroscopy as a function of temperature and pressure for different polarizations. In this original paper, we explore the development and amount of charge disproportionation and the coupling of the electronic degrees of freedom to the counterions and the underlying lattice. The methyl groups undergo changes with temperature that are crucial for the anion cage formed by them. We find that the coupling of the TMTTF molecules to the hexafluorine anions changes upon cooling and especially at the charge-order transition, indicating a distortion of the anion. Additional features are identified that are caused by the anharmonic potential. The spin-Peierls transition entails additional modifications in the charge distribution. To complete the discussion, we also add the vibrational frequencies and eigenvectors based on ab-initio quantum-chemical calculations.
\end{abstract}

Keywords: charge order; spin-Peierls transition; vibrational spectroscopy; infrared spectroscopy; Raman spectroscopy

Classification: PACS 75.25.Dk, 71.20.Ps 74.70.Kn, 78.30.-j, 63.20.-e 


\section{Introduction}

The physics of the one-dimensional organic compounds (TMTCF) ${ }_{2} X$ (with $C=\mathrm{Se}, \mathrm{S}$ and $X$ being a monovalent anion such as $\mathrm{PF}_{6}^{-}, \mathrm{AsF}_{6}^{-}, \mathrm{SbF}_{6}^{-}, \mathrm{Br}^{-}$, or $\mathrm{ClO}_{4}^{-}$) is an active research topic for already three decades [1-8]. The phase diagram presented in Figure 1 is a sort of summary of the findings on these materials. Nevertheless, in recent years it became obvious that some very fundamental issues of the coupling of spin, charge and lattice degrees of freedom - albeit occasionally addressed in some very early papers - are not understood in sufficient depth and require more detailed and comprehensive studies. Especially the charge-ordered state of the Fabre salts (TMTTF) ${ }_{2} X$ still bears some mystery.

Figure 1. The phase diagram of the quasi one-dimensional TMTTF and TMTSF salts, first suggested by Jérome and coworkers [2] and further supplemented by many groups over the years [5]. For the different compounds with centrosymmetric anions the ambient-pressure position in the phase diagram is indicated. Going from the left to the right, the materials get less one-dimensional due to the increasing interaction in the second and third direction. At low temperatures various broken symmetry ground states develop. Here loc stands for charge localization, CO for charge ordering, SP for spin-Peierls, AFM for antiferromagnet, SDW for spin density wave, and SC for superconductor. While some of the boundaries are clear phase transitions, the ones indicated by dashed lines are better characterized as a crossover. The position in the phase diagram can be tuned by external or chemical pressure.

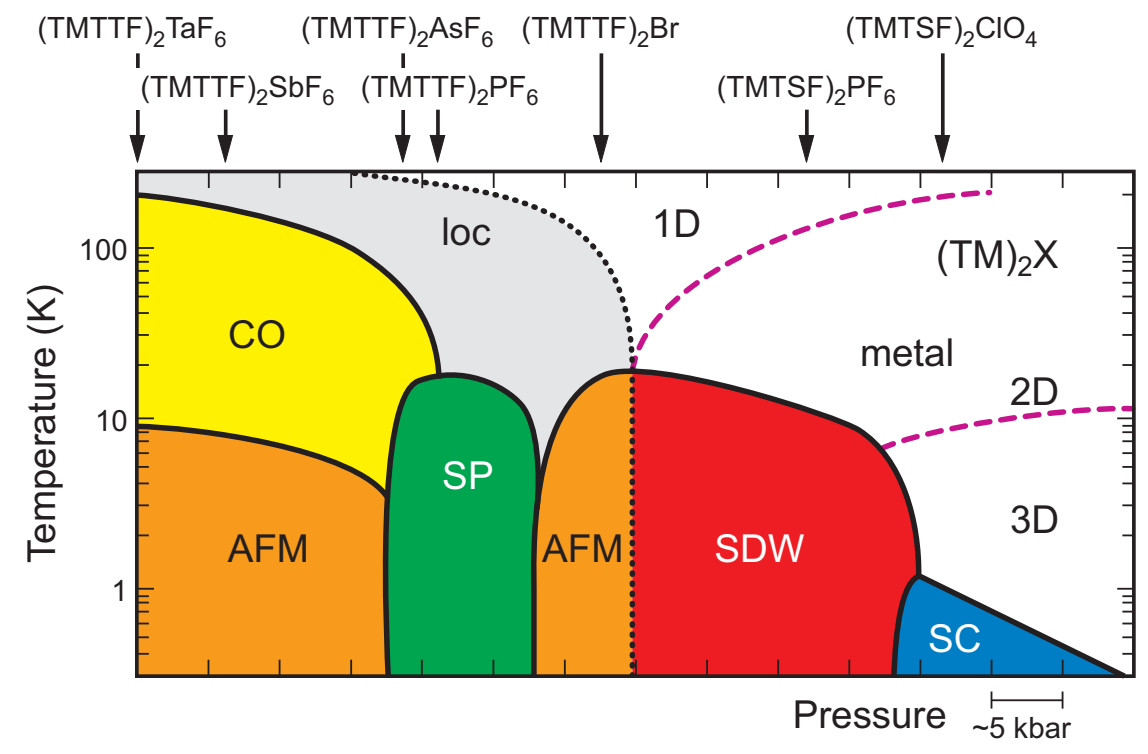

First indications of a phase transition at $T_{\mathrm{CO}}=157 \mathrm{~K}$ in $\left.(\mathrm{TMTTF})\right)_{2} \mathrm{SbF}_{6}$ were provided by thermopower and transport measurements [9], but it took another 15 years before one- and two-dimensional ${ }^{13} \mathrm{C}$ spin labeled NMR spectroscopy could prove charge disproportionation developing for $T<T_{\mathrm{CO}}$ [10-15]. Dielectric permittivity measurements on several of these TMTTF salts finally yielded evidence for ferroelectric behavior of purely electronic origin [16-22]. The ferroelectric state is associated with the charge order and results from the loss of inversion symmetry relating the molecules on the chain. X-ray investigations, however, failed to give evidence for a doubling of the unit cell or other lattice effects associated with that phase transition [23-25]. Calculations based on the extended Hubbard 
Hamiltonian could reproduce the charge-ordered state [26,27], however, the coupling to the lattice-seen by ${ }^{19}$ F NMR spectroscopy [13] or as an anomaly in the thermal expansion [28] -is necessary to describe the complete phase diagram [29-31]. A recent neutron scattering study indicates that charge ordering at $T_{\mathrm{CO}}$ drives a uniform displacement of the anions mainly directed along the $a$-axis [32]. It results in an alternation of charge rich $\left(\rho_{0}+\delta\right)$ and charge poor molecules $\left(\rho_{0}-\delta\right)$. It was proposed that charge order in adjacent stacks occurs in phase, in contrast to the broken translational symmetry observed in other charge-ordered linear chain compounds [33,34]. ESR experiments, however, strongly suggest that in the charge-ordered regime two inequivalent magnetic TMTTF chains coexist [35], most probably due to the loss of translational invariance in the $b c$ plane. The increase of mosaicity around $T_{\mathrm{CO}}[36]$ supports the development of ferroelectric domains in the nanometer scale.

According to stoichiometry, the (TMTTF $)_{2} X$ compounds should form metallic compounds with a three-quarter-filled conduction band, but due to their strong electronic interaction, the systems become insulating upon cooling. Below approximately $250 \mathrm{~K}$ a minimum in resistivity marks a localization of the charge due to on-site Coulomb interaction $U$; the characteristic temperatures are listed in Table 1. Except (TMTTF) ${ }_{2} \mathrm{ClO}_{4}$, all Fabre salts develop a charge-ordered phase below $T<T_{\mathrm{CO}}$, as can be seen by a kink in $\rho(T)$ [Figure 2b] [7,37,38], nuclear magnetic resonance (NMR) [10,11,15,39], electron spin resonance (ESR) [35,40], dielectric [18,20,22], and optical measurements [41-45]. While the magnetic properties at ambient temperature can be described as an antiferromagnetic chain according to the $S=1 / 2$ Heisenberg model, the systems either exhibit a transition to an antiferromagnetic ground state or to a spin-Peierls state at low temperatures (Table 1) [46-48].

Table 1. Transition temperatures for charge localization $T_{\rho}$ and charge order $T_{\mathrm{CO}}$ of various Fabre salts (TMTTF) $)_{2} X . T_{\mathrm{SP}}$ indicates the spin-Peierls transition temperature and $T_{\mathrm{N}}$ is the temperature of the antiferromagnetic ordering, determined by transport and magnetization measurements $[37,46]$.

\begin{tabular}{lcccc}
\hline Compound & $\boldsymbol{T}_{\boldsymbol{\rho}}(\mathbf{K})$ & $\boldsymbol{T}_{\mathbf{C O}}(\mathbf{K})$ & $\boldsymbol{T}_{\mathbf{S P}}(\mathbf{K})$ & $\boldsymbol{T}_{\mathbf{N}}(\mathbf{K})$ \\
\hline$(\mathrm{TMTTF})_{2} \mathrm{PF}_{6}$ & 250 & 67 & 19 & - \\
$(\mathrm{TMTTF})_{2} \mathrm{AsF}_{6}$ & 250 & 102 & 13 & - \\
$(\mathrm{TMTTF})_{2} \mathrm{SbF}_{6}$ & 240 & 157 & - & 8 \\
\hline
\end{tabular}

Charge order is supposed to be driven by the effective Coulomb repulsion $V$ between neighboring molecules with respect to the bandwidth $W=2 t$. Since both parameters depend on the intersite distance, the charge-order transition temperature $T_{\mathrm{CO}}$ can be tuned by applying physical $[13,49-53]$ as well as by chemical pressure [7,37]. As an example, the temperature-dependent resistivity of $(\mathrm{TMTTF})_{2} \mathrm{SbF}_{6}$ is plotted in Figure $2 \mathrm{~b}$ with increasing hydrostatic pressure up to $10 \mathrm{kbar}$. The charge-order transition is seen as a change in slope of $\rho(T)$ that shifts from $T_{\mathrm{CO}}=157 \mathrm{~K}$ at ambient pressure to approximately $125 \mathrm{~K}$ at $5 \mathrm{kbar}$ and well below $100 \mathrm{~K}$ at $10 \mathrm{kbar}$. The weak but crucial coupling between adjacent chains leads to a transverse overlap integral $t_{\perp}$ that significantly influences the electronic properties when approaching the dimensional crossover to a higher-dimensional metal. 
The crystals develop a Drude-like optical response also for $E \| b$ as external or internal pressure increases [54,55]. However, there is no simple and not even a monotonous relation between the interstack distance (given by the unit cell parameter $b$ ) and the charge-ordering transition $T_{\mathrm{CO}}$ [37]. Even more delicate is the interaction between the TMTTF molecules and the anions which are incorporated in a cavity formed by six methyl groups as shown in Figure 2a. In the case of tetrahedral or linear anions with no inversion symmetry, the orientation of the anions becomes ordered as the temperature decreases below $T_{\mathrm{AO}}$. But even for centrosymmetric anions like $\mathrm{PF}_{6}^{-}, \mathrm{AsF}_{6}^{-}$or $\mathrm{SbF}_{6}^{-}$the electronic properties of the compounds are strongly influenced by the link between the sulphur atom on the TMTTF and the ligand of the anions. In Figure $2 \mathrm{c}$ the dependence of $T_{\mathrm{CO}}$ on the shortest distance between the sulfur atoms and the fluorine or oxygen atoms in the anions is plotted for TMTTF salts with different hexaflourines.

Figure 2. (a) Two views on the crystal structure of (TMTTF $)_{2} \mathrm{SbF}_{6}$ illustrate the confinement of the octahedral anions in a cavity formed by methyl groups (gray) of the surrounding TMTTF molecules. Also shown are the closest connections between the fluorine (green) and sulphur atoms (yellow). The right panels demonstrate the shift of the charge-order transition temperature $T_{\mathrm{CO}}$ as a function physical and chemical pressure; (b) Temperature dependence of the DC resistivity of (TMTTF $)_{2} \mathrm{SbF}_{6}$ measured along the $b$-direction at different values of hydrostatic pressure as indicated. $T_{\mathrm{CO}}$ is seen as a kink in $\rho(T)$ that shifts to low temperatures as pressure increases; (c) Dependence of $T_{\mathrm{CO}}$ on the shortest distance between the ligands of the anions ( $\mathrm{F}$ or $\mathrm{O}$ ) and sulfur atoms in TMTTF. The structural data are taken from References [23,56-58]; $c f$. also Reference [37].

(a)
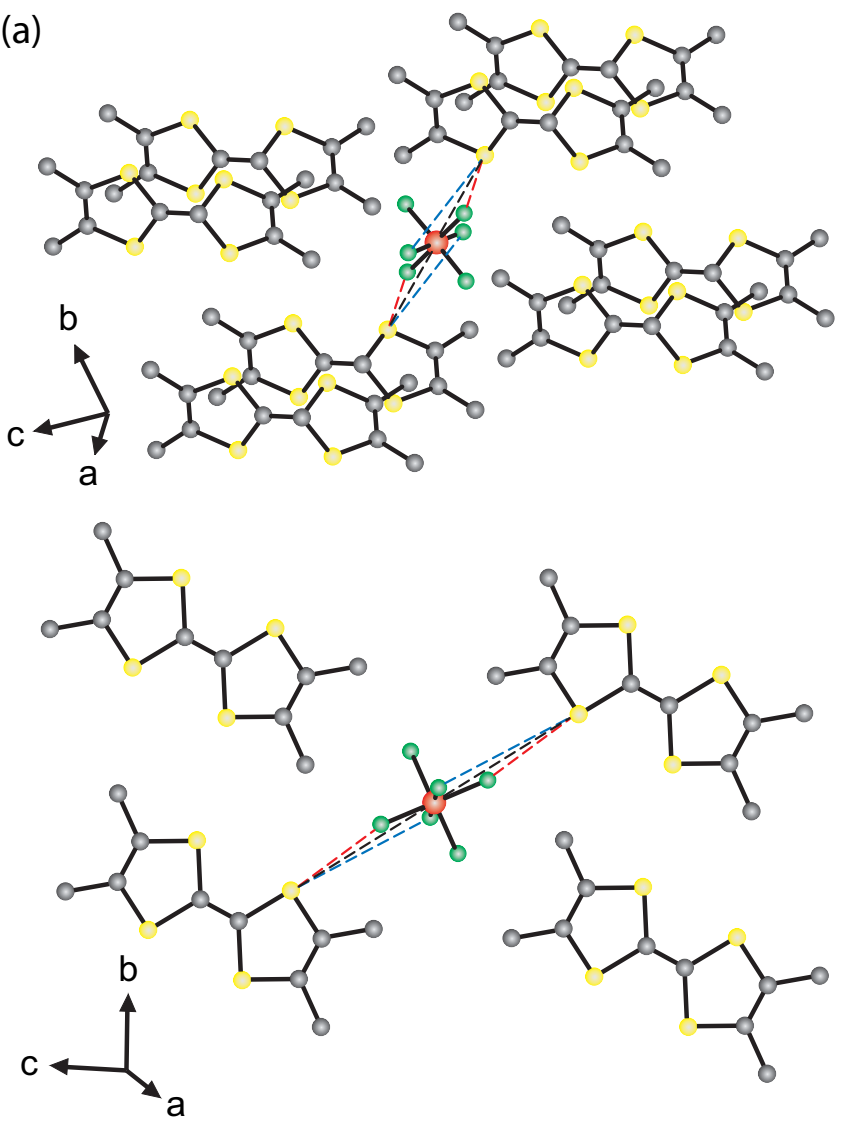
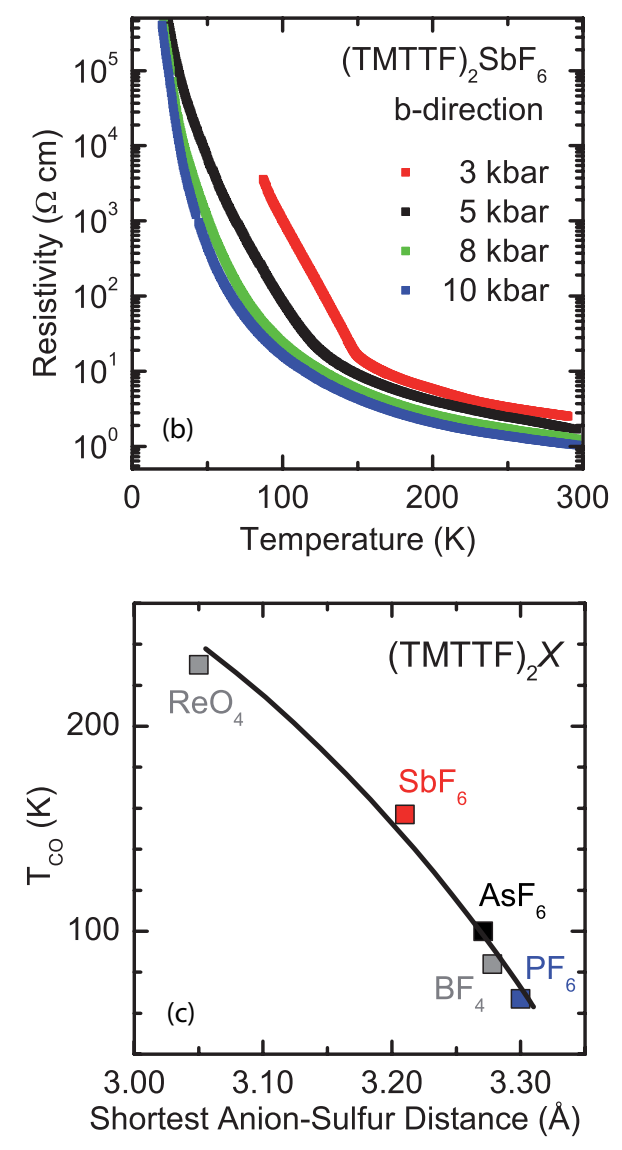
In order to gain more insight into the charge disproportionation of (TMTTF) ${ }_{2} X$ salts, we have performed comprehensive optical investigations of the infrared and Raman active vibrational features around the phase transitions. Most sensitive are the $\nu_{3}\left(a_{g}\right)$ Raman mode and the antisymmetric molecular vibration $\nu_{28}\left(\mathrm{~b}_{1 u}\right)$ probed along the $c$-direction (Figure 3 ). We also observe the low-frequency intermolecular vibrational modes of the TMTTF molecules that are seen in the Raman spectra and become infrared active due to charge disproportionation.

Figure 3. Sketch of the totally symmetric $a_{g}$ modes $\nu_{3}$ and $\nu_{4}$ and the asymmetric $\nu_{28}\left(b_{1 u}\right)$ stretching modes of the TMTTF molecule that mainly involve the central and outer $\mathrm{C}=\mathrm{C}$ bonds. In the right frame, we show the linear shift of the intramolecular vibrations $\nu_{3}\left(a_{g}\right)$ and $\nu_{28}\left(b_{1 u}\right)$ with the charge per TMTTF molecule obtained from optical spectroscopy by Meneghetti et al. [59].
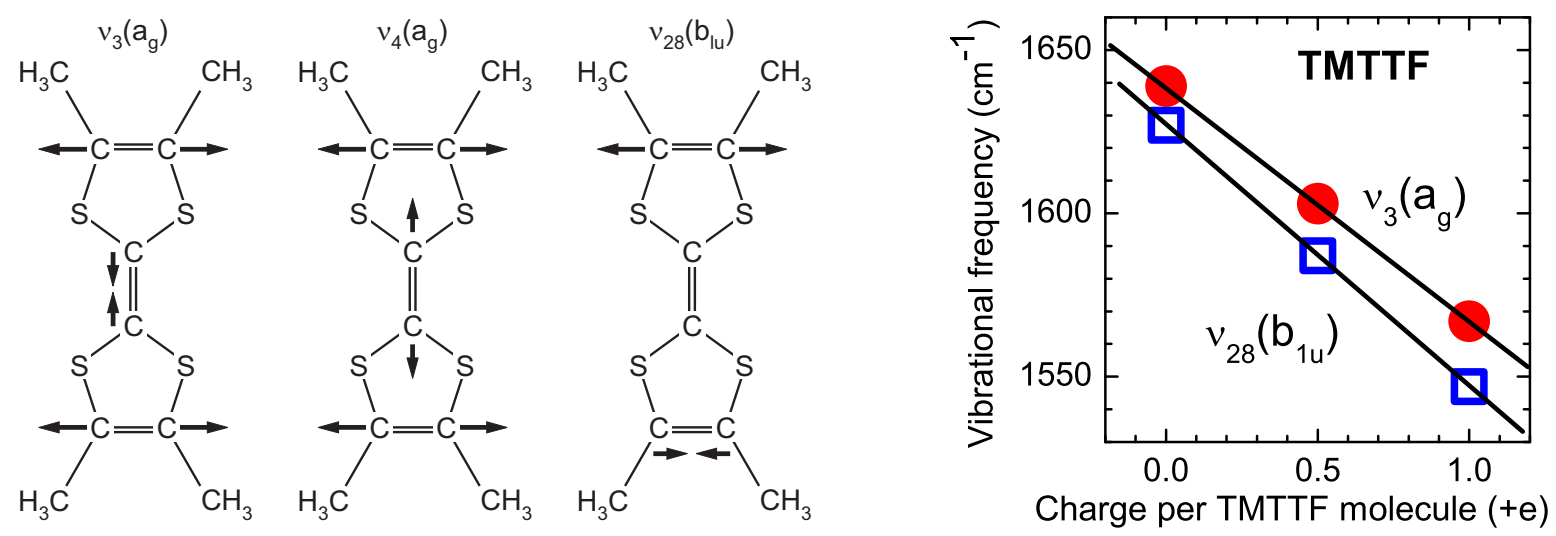

\section{TMTTF Molecule and Vibrational Modes}

In the present paper, we relate our assignments of the vibrational modes to the nomenclature proposed by Meneghetti and coworkers [59] where the molecular structure of TMTTF is assumed to have $\mathrm{D}_{2 h}$ symmetry. According to the character table for the $\mathrm{D}_{2 h}$ point group (Table 2), there are eight different irreducible representations whereas only the antisymmetric $b_{1 u}, b_{2 u}$ and $b_{3 u}$ modes are infrared active and the symmetric $a_{g}, b_{1 g}, b_{2 g}, b_{3 g}$ are Raman active. The $a_{u}$ species is neither Raman nor infrared active. A complete list of the vibrational modes is given in Tables A1 (gerade modes) and A2 (ungerade modes) of the Appendix. Bases on ab-initio quantum-chemical calculations we calculated the frequencies and intensities of the molecular modes of the neutral TMTTF and fully ionized $\mathrm{TMTTF}^{+}$. Of interest are the Raman active $a_{g}$ modes getting also infrared active by electron-molecular vibrational (emv) coupling to the electronic background. They arise in the spectra as Fano-resonances when overlapped to an electronic continuum.

Compared to conventional metals and semiconductors, the building blocks of synthetic metals are large molecules, a fact which has appreciable effects on the vibrational and electronic properties. In general, we can distinguish two sorts of electron-phonon interaction: a coupling to lattice phonons which is normally found in any kinds of solid, and a coupling of the electrons to intramolecular vibrations of the organic molecules which form the conducting layer. Within the framework of the tight-binding description of electrons, the difference between these two effects is that the coupling to lattice vibrations modulates the transfer integrals $t$, while the coupling to intramolecular vibrations modulates the on-site 
energy $\varepsilon$ [60]. The energy of these two kinds of phonons is also distinct: due to the comparably heavy molecules, the lattice modes are observed below approximately $200 \mathrm{~cm}^{-1}$ for these crystals, while the intramolecular vibrations of TMTTF, which show the strongest coupling constants, lie between 500 and $1700 \mathrm{~cm}^{-1}$. In the Appendix we give complete lists of the intramolecular vibrations and their infrared intensities (Tables A1,A2).

Table 2. The analysis of the symmetry species and their polarization-dependent activity for the $\mathrm{D}_{2 h}$ point group of the TMTTF molecule.

\begin{tabular}{|c|c|c|}
\hline \multicolumn{3}{|c|}{$\mathbf{D}_{2 h}$} \\
\hline \multirow{2}{*}{ Symmetry } & \multicolumn{2}{|c|}{ polarization } \\
\hline & Infrared & Raman \\
\hline $\mathrm{a}_{g}$ & & $\mathrm{xx}, \mathrm{yy}, \mathrm{zz}$ \\
\hline $\mathrm{b}_{1 g}$ & & xy \\
\hline $\mathrm{b}_{2 g}$ & & $\mathrm{xz}$ \\
\hline $\mathrm{b}_{3 g}$ & & yz \\
\hline \multicolumn{3}{|l|}{$\mathrm{a}_{u}$} \\
\hline $\mathrm{b}_{1 u}$ & $\mathrm{z}$ & \\
\hline $\mathrm{b}_{2 u}$ & $\mathrm{y}$ & \\
\hline $\mathrm{b}_{3 u}$ & $\mathrm{X}$ & \\
\hline
\end{tabular}

The coupling of electrons to molecular vibrations was first observed and described for one-dimensional systems, but later extended to two dimensions (for a review see for example References [61,62]). The interaction takes place via a modulation of the HOMO energy; for one dimension in the presence of a symmetry break the emv-interaction induces oscillations of the conduction-electron density along the stacking direction with the frequency of the phonons [63-67]; hence the excitations couple to the external infrared radiation. In this context symmetry break means that the molecular units should not lie at the inversion centers. It was shown for one-dimensional systems that if the occupied molecular orbital is non-degenerate, the linear emv-coupling is possible only to the totally-symmetric $a_{g}$ vibrational modes of the molecule [68], which normally are infrared forbidden.

The highest occupied molecular orbital (HOMO) of a neutral TMTTF molecule is drawn in Figure 4 where the highest charge density can be found between the $\mathrm{C}=\mathrm{C}$ double bonds. They are mostly effected by a change of the ionicity of the molecule. These bonds are mainly involved in the vibrational $\nu_{3}, \nu_{4}$ and $\nu_{28}$ modes mentioned in the previous Section 1 (for more details see Appendix). They can be used to detect the onset of the charge ordering as well as a gauge for the charge disproportionation.

Concerning the crystal structure, all Fabre salts are isostructural consisting of stacks of the planar organic molecules TMTTF along the $a$-axis that are separated in the $c$-direction by monovalent anions. In the $b$-direction, the distance of the stacks is comparable to the van der Waals radii. Due to the triclinic symmetry $\mathrm{P} \overline{1}\left(C_{i}\right), b^{\prime}$ denotes the projection of the $b$-axis perpendicular to $a$, and $c^{*}$ is normal to the $a b$-plane. The symmetry analysis for the lattice phonons can be found in Section 5.1. 
Figure 4. Contour plot for the HOMO orbital of a neutral TMTTF molecule. The charge is mainly located on the sulfur atoms and the three $\mathrm{C}=\mathrm{C}$ double bonds.

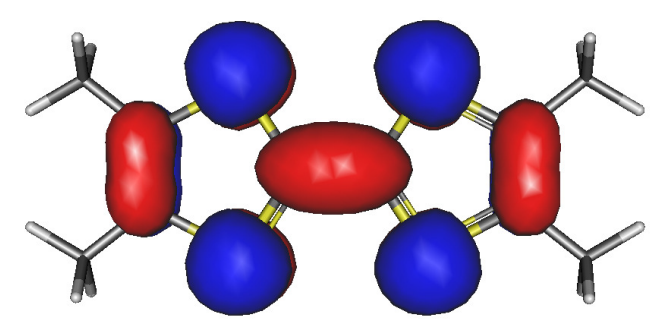

\section{Experimental Details}

Single crystals of the charge-transfer salts (TMTTF) $)_{2} X$ [tetramethyltetrathiafulvalene (TMTTF)] with $X=\mathrm{PF}_{6}^{-}, \mathrm{AsF}_{6}^{-}$, and $\mathrm{SbF}_{6}^{-}$were grown by electrochemical methods as described previously [37,47]. The needle-shaped single crystals are several millimeters long in the $a$-direction and less than a millimeter wide in the other crystal orientations. For our optical investigations we used naturally grown surfaces of single crystals. The in-plane infrared reflection spectra $R(\nu)$ of (TMTTF $)_{2} \mathrm{PF}_{6}$ and $(\mathrm{TMTTF})_{2} \mathrm{AsF}_{6}$ crystals were measured for light polarized along the $a$ and $b^{\prime}$-axes utilizing a Bruker IFS $113 \mathrm{~V}$ Fourier-transform spectrometer in the frequency range $40 \mathrm{~cm}^{-1}<\nu<10,000 \mathrm{~cm}^{-1}$ at temperatures $5 \mathrm{~K}<T<300 \mathrm{~K}$. The crystals were placed either in a He exchange gas cryostat or a cold-finger cryostat. For the reflectivity along the third direction $\left(E \| c^{*}\right)$ we used IR microscope HYPERION attached to a Bruker IFS 66v/s and a Bruker Vertex 80 Fourier transform spectrometer. The microscope is purged with nitrogen to reduce the atmospheric absorption bands. Here the reflectivity spectra were collected in a frequency range from 500 to $8000 \mathrm{~cm}^{-1}$ with a resolution of $0.5 \mathrm{~cm}^{-1}$ at temperatures from 290 down to $10 \mathrm{~K}$. From the frequency dependent reflectivity we derived the real part of the optical conductivity $\sigma_{1}(\nu)$ and the dielectric loss $\epsilon_{2}(\nu)$ by Kramers-Kronig analysis, using our DC resistivity data [37] for the low-frequency extrapolation and previously published data on (TMTTF $)_{2} \mathrm{PF}_{6}$ for the high-frequency extrapolation [69-72]. More details on the data analysis can be found in References [62,73].

Raman spectra on (TMTTF $)_{2} X\left(X=\mathrm{PF}_{6}^{-}, \mathrm{AsF}_{6}^{-}\right)$single crystals have been measured using four different excitation energies: the $\lambda=568,647,676$ and $752 \mathrm{~nm}$ line from a $\mathrm{Kr}^{+}$laser. Laser line was focused onto a smooth part of the crystal by a $20 \times$ magnification objective and the scattered radiation was analyzed with a Renishaw 1000 spectrometer equipped with ultra-steep long-pass filter. Next premonochromator has been used for low-frequency spectra. The 647 and the $676 \mathrm{~nm}$ lines are almost resonant with the first localized electronic excitation of the (TMTTF) ${ }_{2}{ }^{+}$system occurring around $15,000 \mathrm{~cm}^{-1}$ in the perpendicular polarization ( $b^{\prime}$-direction) [70]. The 568 and $752 \mathrm{~nm}$ lines are both out of resonance, and in particular the laser line at $752 \mathrm{~nm}$ can be used to study polarization dependence and to measure Raman scattering excited with light polarized along the stack ( $a$-axis) due to the very low background signal. The $568 \mathrm{~nm}$ line has the serious drawback to be strongly resonant with the $\mathrm{TMTTF}^{+}$ cationic species [74], therefore spectra are totally dominated by $\mathrm{TMTTF}^{+}$bands probably originating from impurities crystallized on the sample surface. For temperature dependent measurements, the crystals were attached to a cold finger cryostat. Heating of the sample by laser irradiation can amount to as much as $10 \mathrm{~K}$ at low temperatures; it was accounted by for recalibration. 


\section{Molecular Vibrations}

The spectral range from 200 to $3000 \mathrm{~cm}^{-1}$ is dominated by intramolecular vibrations of the TMTTF molecules and of the hexafluoride anions. The modes involving the covalent $\mathrm{C}=\mathrm{C}$ bonds are most sensitive for the charge on the molecules and will be used to obtain information about the charge disproportionation. The vibrations of the terminal methyl groups give indications on the coupling between the anions and the TMTTF molecules via the weak $\mathrm{C}-\mathrm{H} \cdots \mathrm{F}$ interactions. Also the vibrational modes of the anions are changed by distortion of the octahedrons.

\subsection{High-Energy Raman Spectra: Molecular Vibrations}

Raman spectra excited with the $647 \mathrm{~nm}$ line have been measured with the incident light polarized perpendicular to the $a$-axis to fully exploit resonance effects. Figure 5a contains the room temperature spectra of $(\mathrm{TMTTF})_{2} \mathrm{PF}_{6}$ and $(\mathrm{TMTTF})_{2} \mathrm{AsF}_{6}$. They are characterized by a strong fluorescence background and by the three most strongly coupled molecular vibrations, $\nu_{3}\left(a_{g}\right)=1599 \mathrm{~cm}^{-1}$, $\nu_{4}\left(a_{g}\right)=1474 \mathrm{~cm}^{-1}$ and $\nu_{10}\left(a_{g}\right)=502 \mathrm{~cm}^{-1}$. In addition we can identify an overtone of the $\nu_{10}\left(a_{g}\right)$ mode around $1000 \mathrm{~cm}^{-1}$ and a shoulder on the low energy side of the $\nu_{3}\left(a_{g}\right)$ band that could be assigned to the $\nu_{28}\left(b_{1 u}\right)=1580 \mathrm{~cm}^{-1}$ molecular mode.

In the right panel of Figure 5 the polarization-dependent Raman spectra are presented as measured with the $\lambda=752 \mathrm{~nm}$ laser line. The $\left(b^{\prime}, b^{\prime}\right)$-polarization spectrum (incident and scattered light both polarized perpendicular to the $a$-axis) is characterized by the same three coupled molecular vibrations present in the $647 \mathrm{~nm}$ spectra, while these bands are totally absent in the $(a, a)$-polarization (incident and scattered light both polarized parallel to the $a$-axis).

Figure 5. (a) Room temperature Raman spectra of (TMTTF $)_{2} X\left(X=\mathrm{PF}_{6}^{-}, \mathrm{AsF}_{6}^{-}\right)$single crystals measured with the $\lambda=647 \mathrm{~nm}$; (b) Polarized room temperature Raman spectra of $(\mathrm{TMTTF})_{2} \mathrm{AsF}_{6}$ measured with the $\lambda=752 \mathrm{~nm}$. In parentheses the components of the polarizations tensor probed in the experiment.
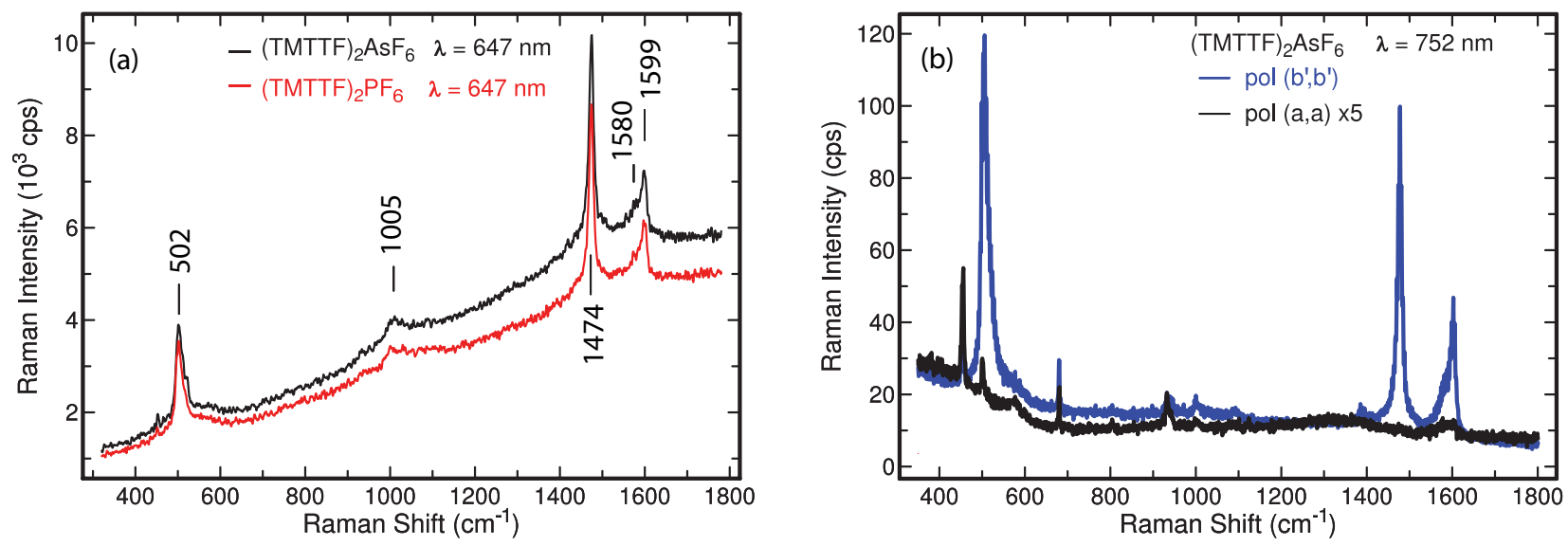

In both polarization directions in the range between $800 \mathrm{~cm}^{-1}$ and $1000 \mathrm{~cm}^{-1}$ several small peaks occur which can be assigned to combination and overtone modes. The weak peak at $800 \mathrm{~cm}^{-1} \mathrm{can}$ be assigned to the combination of the $\nu_{10}\left(a_{g}\right)$ and the $\nu_{11}\left(a_{g}\right)$ modes whereas the feature at around 
$1000 \mathrm{~cm}^{-1}$ is related to the aforementioned first overtone of $\nu_{10}\left(a_{g}\right)$. The intermediate mode at $930 \mathrm{~cm}^{-1}$ can be identified as the $\nu_{8}\left(a_{g}\right)$ mode. A weak and very broad spectral feature can also be identified in the (a, a)-polarization around $1300 \mathrm{~cm}^{-1}$ : its shape and bandwidth seem to indicate a vibronic or electronic origin, however its assignment to the vibronic anti-phase combination of $\nu_{4}\left(a_{g}\right)$ modes is questionable due to symmetry arguments and energy difference with the corresponding infrared signal (where it shows up at around $1200 \mathrm{~cm}^{-1}$ for the polarization $E \| a$ ).

In order to investigate the charge disproportionation among the TMTTF molecules at the charge-ordering transition, we have performed Raman experiments on (TMTTF $)_{2} \mathrm{PF}_{6}$ and $(\mathrm{TMTTF})_{2} \mathrm{AsF}_{6}$ as a function of temperature. In Figure 6 we display the temperature evolution of the (TMTTF $)_{2} \mathrm{AsF}_{6}$ Raman spectra measured with the $647 \mathrm{~nm}$ laser line. The splitting of the $\nu_{3}\left(a_{g}\right)$ mode can be clearly seen below $T=100 \mathrm{~K}$; it is a fingerprint of the disproportionation of charge between the two TMTTF molecules in the unit cell.

In Raman spectra, the $\nu_{3}\left(a_{g}\right)$ mode is the best choice to gain information on the molecular charge distribution since it shows a large and linear ionicity shift and is only weakly coupled to the electronic system [59,75]; in Figure 3 the shift of the vibrational frequency is plotted as a function of (positive) charge per TMTTF molecule. On the contrary the $\nu_{4}\left(a_{g}\right)$ mode, which has the strongest e-ph coupling constant, cannot provide a reliable estimate of the ionicity in charge-ordered non-centrosymmetric one-dimensional systems [76]. Particularly, the $\nu_{4}\left(a_{g}\right)$ mode in our spectra of (TMTTF $)_{2} \mathrm{AsF}_{6}$ and (TMTTF) ${ }_{2} \mathrm{PF}_{6}$ (Figures 6,7) remains almost fixed around $1479 \mathrm{~cm}^{-1}$, which corresponds to an average charge of $\rho_{0}=+0.5 e$, and does not show any splitting. Yamamoto and Yakushi explained these phenomena as the combined effects of the electron-phonon perturbation and of the ionicity shift $[77,78]$.

Figure 6. Raman spectra of (TMTTF $)_{2} \mathrm{AsF}_{6}$ as a function of temperature. The data are taken with $\lambda=647.2 \mathrm{~nm}$ for the polarization $\left(b^{\prime}, b^{\prime}\right)$. Most obvious is the splitting of the $\nu_{3}\left(a_{g}\right)$ mode around $1600 \mathrm{~cm}^{-1}$ and the evolution of three features between 1425 and $1460 \mathrm{~cm}^{-1}$ as the temperatures drops below $T_{\mathrm{CO}}=102 \mathrm{~K}$. For $T=95 \mathrm{~K}$ the two Lorentzian contributions are plotted separately to demonstrate the decomposition.

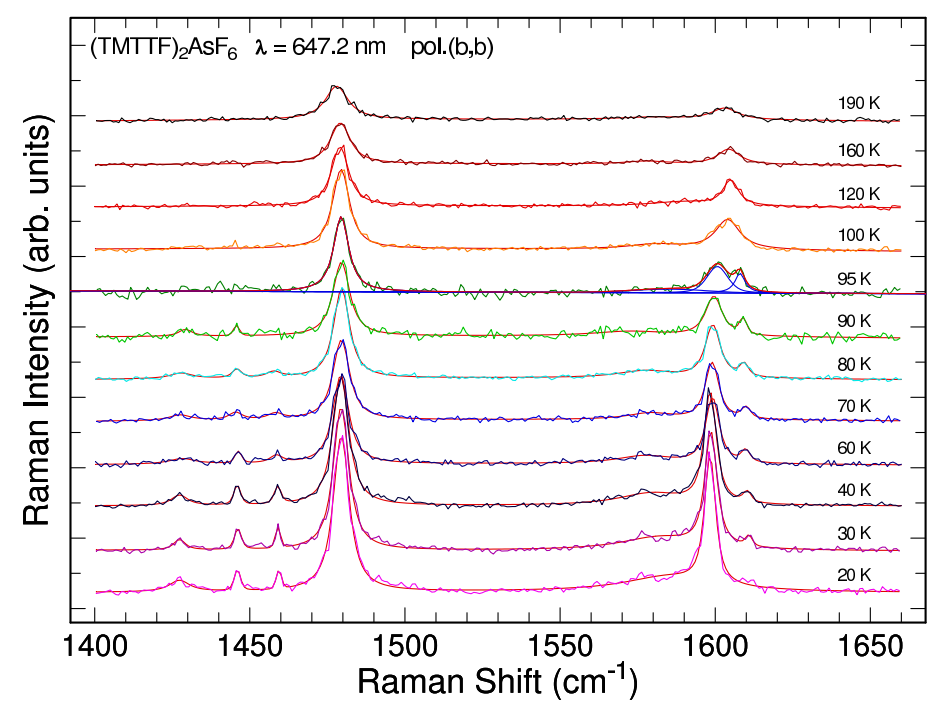


Figure 7. Raman spectra of (TMTTF $)_{2} \mathrm{PF}_{6}$ as a function of temperature. Similar to the spectra of (TMTTF $)_{2} \mathrm{AsF}_{6}$, the $\nu_{3}\left(a_{g}\right)$ mode around $1600 \mathrm{~cm}^{-1}$ splits as the temperature drops below $T_{\mathrm{CO}}=67 \mathrm{~K}$ and three features evolve between 1425 and $1460 \mathrm{~cm}^{-1}$ that can be assigned to bending modes of the methyl groups. For $T=13 \mathrm{~K}$ we plot the different contributions separately.

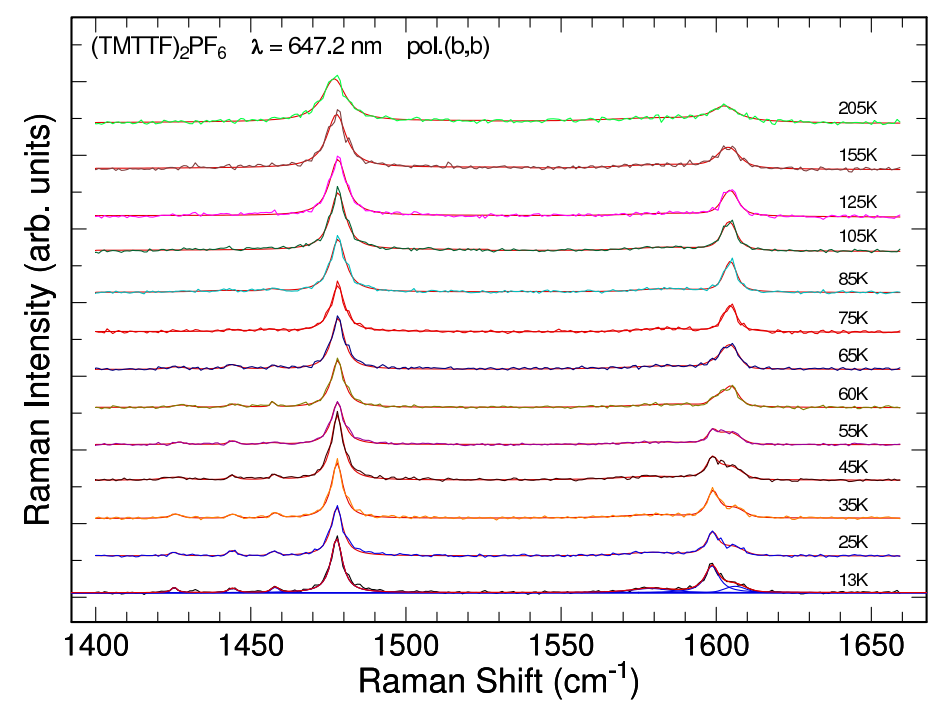

Figure 8. Left two panels: Temperature evolution of the peak frequencies of the $\nu_{3}\left(a_{g}\right)$ mode of (TMTTF) $)_{2} X$ obtained from our Raman (open circles) and infrared spectra (filled squares). The corresponding charge disproportionation $2 \delta$ is plotted in the right panel as a function of temperature.
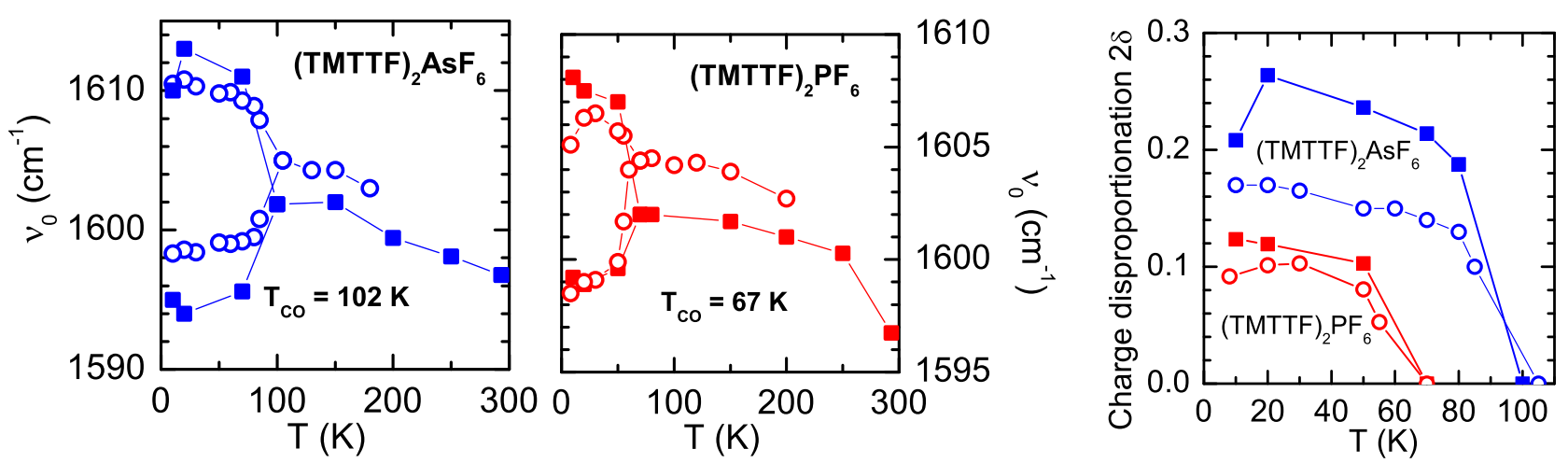

A careful inspection of our (TMTTF $)_{2} \mathrm{AsF}_{6}$ spectra evidences that the $\nu_{3}\left(a_{g}\right)$ band gains intensity on lowering the temperature; down to $T=120 \mathrm{~K}$ the band slightly narrows, but then broadens when cooled further to $100 \mathrm{~K}$. At that point the band splits abruptly reaching $\Delta \nu=7 \mathrm{~cm}^{-1}$ already at $T=95 \mathrm{~K}$. As demonstrated in Figure 8, the amplitude of the splitting increases slightly on lowering the temperature further and reaches its maximum value of $\Delta \nu=12 \mathrm{~cm}^{-1}$ at $30 \mathrm{~K}$. According to

$$
2 \delta=\frac{\Delta \nu}{72 \mathrm{~cm}^{-1} / e}
$$


obtained from Figure 3 the charge imbalance is calculated to $\rho_{\text {rich }}-\rho_{\text {poor }}=2 \delta=0.17 e$ for $(\mathrm{TMTTF})_{2} \mathrm{AsF}_{6}$. There are interesting differences of how the intensity of the two components assigned to the $\nu_{3}\left(a_{g}\right)$ mode evolves with temperature. Both exhibit comparable intensities in the $85 \mathrm{~K}$ spectrum right below $T_{\mathrm{CO}}$; upon cooling the lower-energy component becomes stronger, while the higher-energy suddenly loses intensity and almost disappears at the lowest temperature (Figure 6).

This behavior is anomalous because in the framework of centrosymmetric dimeric system the higher-energy component should correspond to the Raman-active in-phase mode combination, while the lower energy to the infrared-active out-of-phase combination. Obviously charge order breaks the inversion symmetry, nevertheless for a small charge imbalance these general considerations should remain valid; i.e., the higher-energy component should have the higher Raman intensity.

In Figure 7 the Raman spectra of $(\mathrm{TMTTF})_{2} \mathrm{PF}_{6}$ are shown for various temperatures as indicated. The observations are very similar as for the (TMTTF $)_{2} \mathrm{AsF}_{6}$ salt including the anomalous intensity evolution of the $\nu_{3}\left(a_{g}\right)$ features. In the case of (TMTTF $)_{2} \mathrm{PF}_{6}$ the $\nu_{3}\left(a_{g}\right)$ mode splits between $T=70$ and $65 \mathrm{~K}$; it grows from $4 \mathrm{~cm}^{-1}$ to $7.5 \mathrm{~cm}^{-1}$ when cooled down to $20 \mathrm{~K}$ (corresponding to $2 \delta=0.10 e$, according to Equation 1), suffering a small flection at the lowest temperatures (see Figure 8) due to the spin-Peierls transition at $T_{\mathrm{SP}}=19 \mathrm{~K}$. We will come back to this point in Section 6.

Figure 9. Raman spectra of (TMTTF $)_{2} \mathrm{AsF}_{6}$ and $(\mathrm{TMTTF})_{2} \mathrm{PF}_{6}$ as a function of temperature. The spectral features observed on the low-frequency wing of the strong $\nu_{3}\left(a_{g}\right)$ band is assigned to the molecular $\nu_{28}\left(b_{1 u}\right)$ mode and followed by the solid line. The decomposition of the $T=20 \mathrm{~K}$ spectrum illustrates the different contributions to the fit.
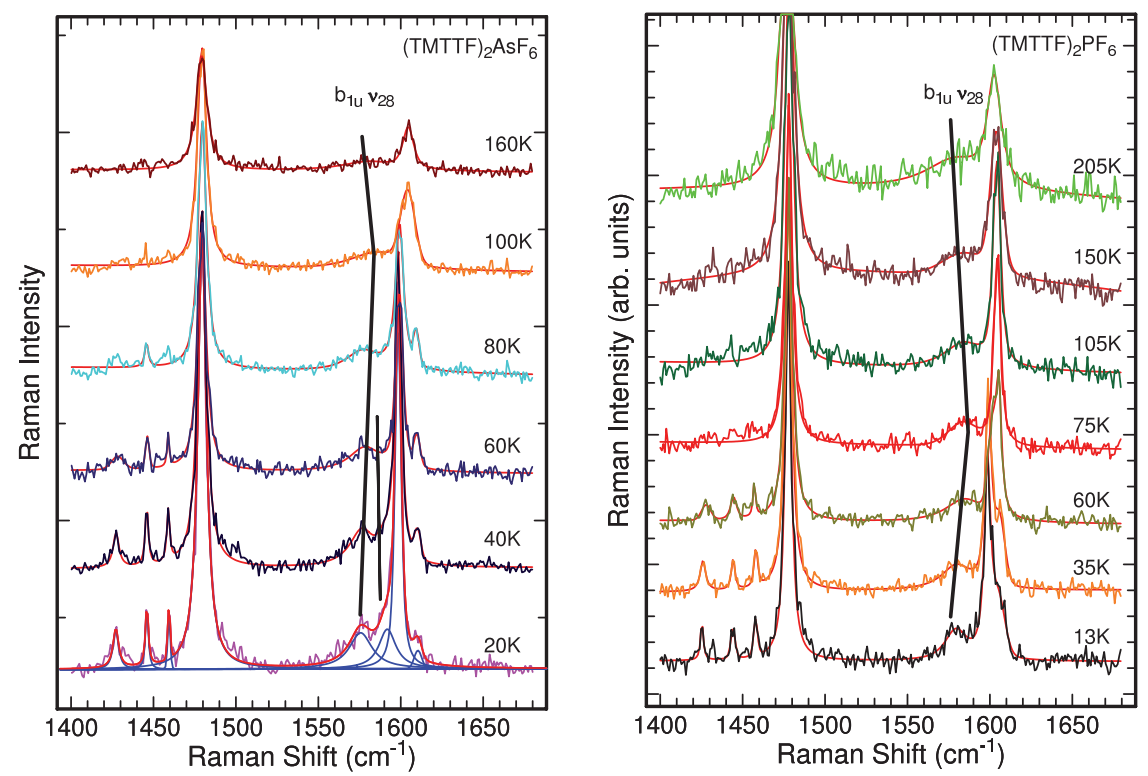

Two observations should be noted at this point: In both compounds a weak spectral feature occurs around $1580 \mathrm{~cm}^{-1}$ which we assign to the Raman-active anti-phase combination of the $\nu_{28}\left(b_{1 u}\right)$ mode; its temperature dependence can be followed in Figure 9. This mode is very sensitive to the charge distribution in TMTTF systems and can best be followed by infrared measurements performed with the polarization $E \| c$, as discussed in more detail in Section 4.2. From Figure 10 it is seen that upon cooling, the mode first shifts to higher frequencies, before it splits at $T_{\mathrm{CO}}$. The stronger low-energy peak 
then softens as the temperature decreases further. Unfortunately it is quite hard to follow the frequency evolution and splitting of this $\nu_{28}\left(b_{1 u}\right)$ mode in our Raman data, since it appears as a shoulder in the high temperature spectra and exhibits only a weak and broad profile at low temperature. Nevertheless its identification by Raman measurements offers a useful internal check for our infrared analysis, presented in the the following Section 4.2.

Figure 10. The optical conductivity of $(\mathrm{TMTTF})_{2} X$ with $X=\mathrm{PF}_{6}^{-}\left(T_{\mathrm{CO}}=67 \mathrm{~K}\right), \mathrm{AsF}_{6}^{-}$ $\left(T_{\mathrm{CO}}=102 \mathrm{~K}\right)$ and $\mathrm{SbF}_{6}^{-}\left(T_{\mathrm{CO}}=157 \mathrm{~K}\right)$ recorded along the $c$-direction for different temperatures as indicated. The conductivity was shifted by a constant factor for better clarification. Below $T_{\mathrm{CO}}$, the $\nu_{28}\left(b_{1 u}\right)$ mode splits in two components as shown by the two black arrows. It is interesting to note that in the case of (TMTTF) $)_{2} \mathrm{SbF}_{6}$ the modes have a strong Fano shape.

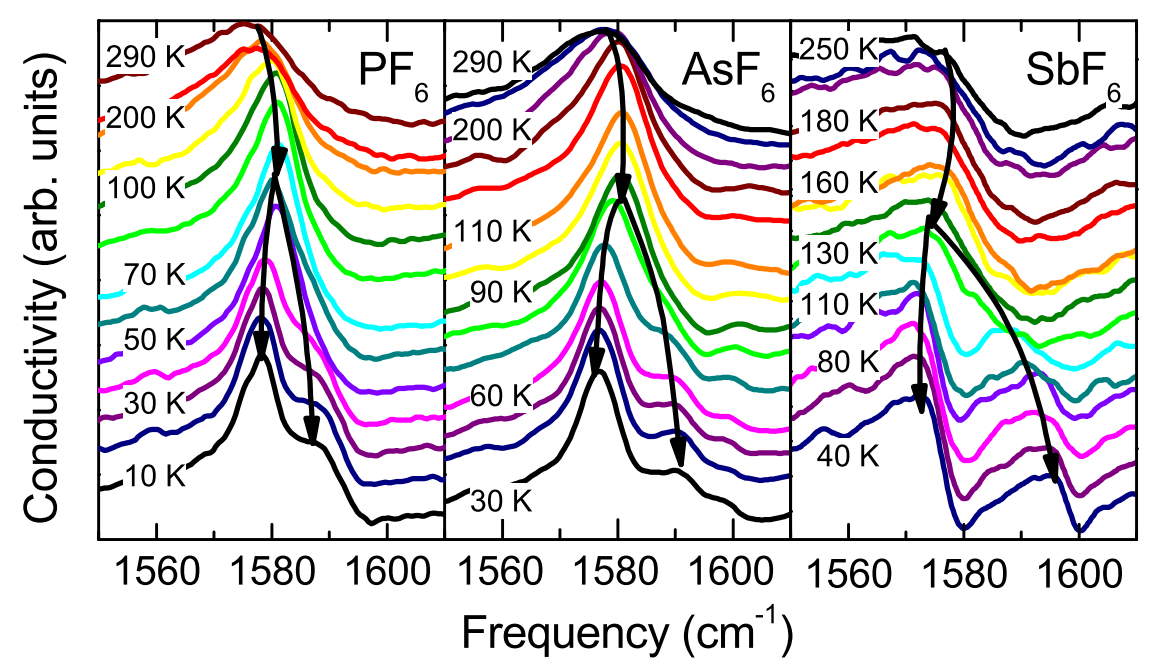

The second observation refers to the three clear and sharp bands that occur at 1427, 1446 and $1459 \mathrm{~cm}^{-1}$ in the charge-ordered state. They can be safely assigned to $\mathrm{CH}$-bending mode of methyl groups and will be discussed in more detail in Section 4.3. Their appearance below $T_{\mathrm{CO}}$ implies some kind of ordering of the TMTTF methyl groups due to the interaction with the anions which could possibly stabilize the $4 k_{F}$-charge-density-wave charge-ordered state [79].

\subsection{Mid-Infrared Vibrational Spectroscopy of Charge-Sensitive Modes}

First indications for charge disproportionation from optical measurements have been obtained by in-plane reflection measurements [42-44]. In Figure 11 the optical conductivity of (TMTTF $)_{2} \mathrm{AsF}_{6}$ and (TMTTF $)_{2} \mathrm{PF}_{6}$ is plotted for different temperatures as indicated. The data are obtained from reflection experiments performed on the $a b$-plane that are dominated by the response of the conduction electrons. At elevated temperatures, a dip is observed around $1600 \mathrm{~cm}^{-1}$ that is caused by the electron-molecular vibrational (emv) coupled $\nu_{3}\left(a_{g}\right)$ mode. This Raman mode is not infrared active by itself, but via coupling to the electronic background it becomes visible as an antiresonance in the polarization along the stacking direction. The temperature dependence of the minima (indicated by arrows in Figure 11) is plotted in Figure 8 together with the Raman peaks. In the right panel also the corresponding charge disproportionation of (TMTTF $)_{2} \mathrm{AsF}_{6}$ and $(\mathrm{TMTTF})_{2} \mathrm{PF}_{6}$ is plotted as a function of temperature. 
Figure 11. Mid-infrared conductivity $\sigma_{1}(\nu)$ of $(\mathrm{TMTTF})_{2} \mathrm{AsF}_{6}$ and $(\mathrm{TMTTF})_{2} \mathrm{PF}_{6}$ for light polarized parallel to the molecular stacks $(E \| a)$ in the spectral region of the emv coupled totally symmetric intramolecular $\nu_{3}\left(a_{g}\right)$ mode for temperatures above and below $T_{\mathrm{CO}}$. The curves are displaced by a constant factor to avoid overlap. The arrows indicate the positions of the antiresonance dips.
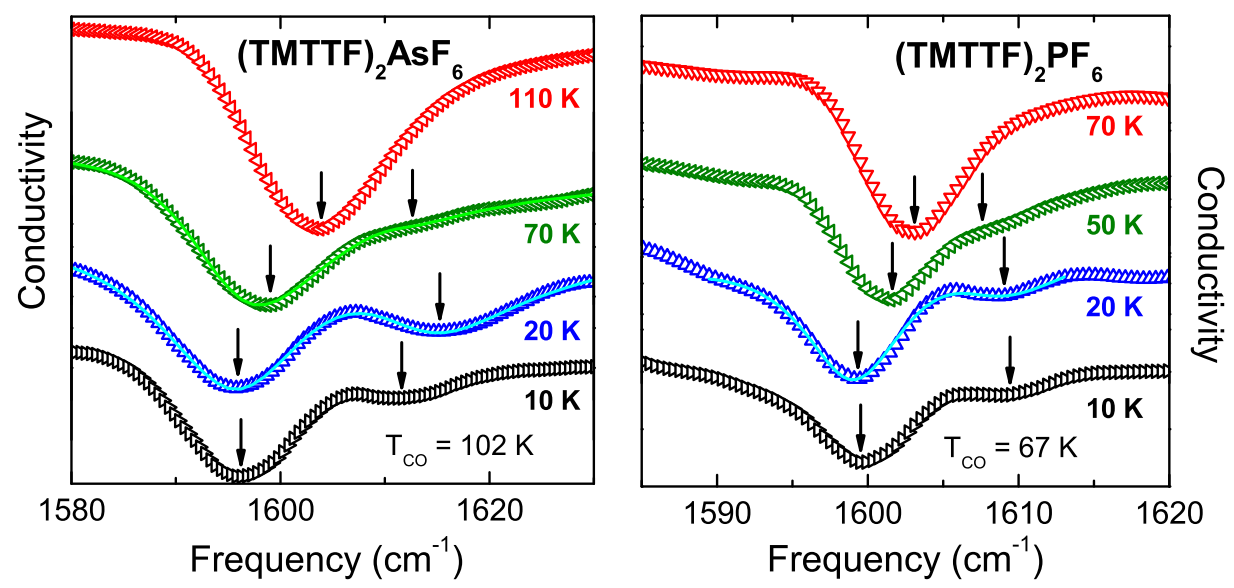

As pointed out by several scholars $[41,59,80,81]$, the antisymmetric $\nu_{28}\left(b_{1 u}\right)$ stretching mode is probably the most sensitive local probe for charge disproportionation as it involves the outer $\mathrm{C}=\mathrm{C}$ bonds of the fulvalene rings where most of the charge is accumulated as visualized in Figure 4. Although Hirose et al. were able to observe this mode in their in-plane reflection measurements [41], a better and more direct approach consists in measuring in the perpendicular direction, i.e., $E \| c$ where the electric field is mainly along the molecular axis and the background signal due to conduction electrons is lower.

In the optical conductivity presented in Figure 10 a strong mode is seen that splits as the temperature drops below $T_{\mathrm{CO}}$. The peaks can be assigned to charge-rich $\left(\rho_{0}+\delta\right)$ and charge-poor $\left(\rho_{0}-\delta\right)$ TMTTF molecules. There is a very good agreement with the weak $\nu_{28}\left(b_{1 u}\right)$ feature observed in the Raman spectra (Figure 9), as far as the frequencies and the temperature dependence is concerned.

The analysis of the spectral weight and width of the modes observed in the infrared spectra is not straightforward, since the modes overlap, are strongly distorted and are influenced by the electronic contributions. Nevertheless, in our spectra the intensity of the lower-frequency peak is always stronger than the high-frequency one due to the higher intrinsic intensity of this mode in the cationic $\mathrm{TMTTF}^{+}$ species. A comprehensive analysis has been performed for the two-dimensional BEDT-TTF conductors and superconductors $[82,83]$ that in principle could also be applied to all systems based on TTF moiety and its derivatives.

Following Meneghetti et al. [59], the resonance frequency of the $\nu_{28}\left(b_{1 u}\right)$ mode occurs at $1627 \mathrm{~cm}^{-1}$ for neutral TMTTF and at $1547 \mathrm{~cm}^{-1}$ for $\mathrm{TMTTF}^{+}$(cf. Table A2). Assuming a linear shift of the resonance frequency with the ionicity of the molecule, the charge disproportionation $2 \delta$ can be calculated from the difference $\Delta \nu$ of the resonance frequencies:

$$
2 \delta=\frac{\Delta \nu}{80 \mathrm{~cm}^{-1} / e}
$$

In Figure 12 the temperature dependence of the peak position and the charge disproportionation is plotted for (TMTTF $)_{2} \mathrm{PF}_{6}$, (TMTTF $)_{2} \mathrm{AsF}_{6}$ and (TMTTF) $)_{2} \mathrm{SbF}_{6}$. The low temperature values are listed 
in Table 3. Two interesting observations should be noted: (i) if the charge imbalance $2 \rho(T)$ is taken as a sort of order parameter, its temperature dependence basically follows the mean-field behavior for $(\mathrm{TMTTF})_{2} \mathrm{PF}_{6}$ while it becomes more abrupt when going to (TMTTF) ${ }_{2} \mathrm{SbF}_{6}$ with the tendency towards a first-order transition. From a broad maximum in $\epsilon_{1}(T)$ observed in the $35-50 \mathrm{~K}$ temperature range [16], the possibility of local charge order and/or ferroelectric domains has been inferred [84]. In our experiments we do not see any indications of an additional phase transition down to $T=20 \mathrm{~K}$; (ii) In all three salts, the lower frequency peak of the $\nu_{28}\left(b_{1 u}\right)$ mode pair rapidly moves towards lower frequency due to the continuous depletion of charge, but then the peak position reverses its temperature behavior and slightly shifts toward higher frequencies. The effects seems to be beyond what might be explained by thermal contraction. Interestingly, the temperature dependence of the split totally symmetric $\nu_{3}\left(a_{g}\right)$ mode does not exhibit such a behavior, as demonstrated in Figure 8.

Figure 12. (a) Position of the peaks for the (TMTTF) $)_{2} X$ with $X=\mathrm{SbF}_{6}^{-}, \mathrm{AsF}_{6}^{-}$and $\mathrm{PF}_{6}^{-}$ as a function of $T$ recorded along the $c$ axis; (b) Temperature dependence of the charge dispropotionation probed by the splitting of the $\nu_{28}$ mode. The charge imbalance $2 \delta$ is estimated from the difference of the two resonance frequencies by Equation 2.
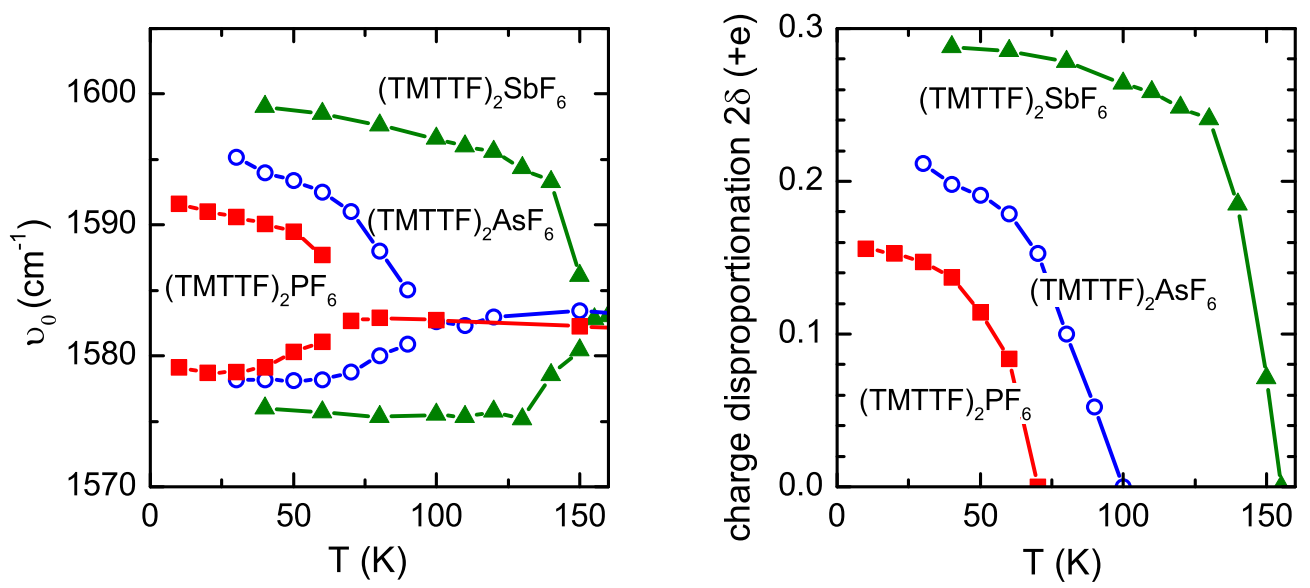

Table 3. The charge disproportionation $2 \delta$ is defined as the difference between charge rich site $\rho_{0}+\delta$ and charge poor sites $\rho_{0}-\delta$. First estimates are based on magnetic measurements; here we add the values obtained from our infrared and Raman investigations conducted at low temperatures as indicated in brackets. $2 \nu_{10}\left(a_{g}\right)$ indicates the first harmonic of the totally symmetric $\nu_{10}$ mode that appears around $1000 \mathrm{~cm}^{-1}$.

\begin{tabular}{|c|c|c|c|c|c|}
\hline \multirow{2}{*}{ Compound } & \multicolumn{5}{|c|}{$2 \delta[\mathrm{e}]$} \\
\hline & NMR & $\nu_{28}\left(b_{1 u}\right)$ IR & $2 \nu_{10}\left(a_{g}\right) \operatorname{IR}$ & $\nu_{3}\left(a_{g}\right)$ IR & $\nu_{3}\left(a_{g}\right)$ Raman \\
\hline$(\mathrm{TMTTF})_{2} \mathrm{PF}_{6}$ & 0.28 [39] & $0.15(10 \mathrm{~K})$ & $0.14(10 \mathrm{~K})$ & $0.12(30 \mathrm{~K})$ & $0.10(20 \mathrm{~K})$ \\
\hline \multirow[t]{3}{*}{$(\mathrm{TMTTF})_{2} \mathrm{AsF}_{6}$} & $0.33[15]$ & $0.21(30 \mathrm{~K})$ & $0.19(30 \mathrm{~K})$ & $0.26(20 \mathrm{~K})$ & $0.17(20 \mathrm{~K})$ \\
\hline & $0.50[11]$ & & & & \\
\hline & $0.16[41]$ & $0.11[41]$ & & & \\
\hline$(\mathrm{TMTTF})_{2} \mathrm{SbF}_{6}$ & 0.50 [12] & $0.29(40 \mathrm{~K})$ & $0.26(40 \mathrm{~K})$ & & \\
\hline
\end{tabular}


Finally, we would like to point out that our local probe of the $\mathrm{C}=\mathrm{C}$ bonds is a direct measure of the charge per TMTTF molecule; thus the plots in Figure 8 and Figure 12 give the temperature dependence of the charge-order amplitude, which can be considered as the order parameter of this phase. In contrast to the results inferred from ${ }^{13} \mathrm{C}-\mathrm{NMR}$ spectra [13], we do not see any suppression of the charge disproportionation at lower temperatures. However, looking at the temperature dependence of the $\nu_{3}\left(a_{g}\right)$ mode displayed in Figure 6 for the example of $(\mathrm{TMTTF})_{2} \mathrm{AsF}_{6}$, we can see that the intensity of the high-frequency peak gets weaker on the expense of the low-frequency peak as the temperature decreases. At $T=20 \mathrm{~K}$ is is only visible as a shoulder. The intensity of the charge-order-split modes is discussed by Girlando in detail [82] and it would be worth to dwell on this issue further.

\subsection{Methyl Groups}

There is an ongoing discussion about the involvement of the anions in the charge-order transition of TMTTF and BEDT-TTF salts and about further changes of the lattice [85-87]. The anions are located in a cavity formed by the methyl groups of the adjacent TMTTF molecules, thus we expect that the pure methyl vibrations are most sensitive to changes of the anions in that cage due to the sizeable $\mathrm{F} \cdots \mathrm{H}$ interaction. Already in 1984 Kistenmacher discussed the role of the cavity and anion size and their interaction for the isostructural TMTSF salts $[88,89]$. There are four methyl groups closest to the anions which are located in the $b^{\prime} c^{*}$-plane, and another two in the $a c^{*}$-plane. These six methyl groups determine three sets of three-fold symmetry axes close to the symmetry axes of the octahedron. The four closest $\mathrm{CH}_{3}$ groups delimit two sets of two-fold symmetry axes which are close to the axes of the octahedron. In addition, the interaction to the fluorine atoms form weak hydrogen bond networks with the closest methyl groups (Figure 2) that become particularly effective at low temperatures [90,91].

Two effects are envisioned: (i) the motion of the anions slows down as the temperature is reduced and eventually it locks into a fixed position. This seems to be rather a pre-requirement of charge order than a consequence; (ii) The charge disproportionation among the TMTTF molecules modifies the interaction between the organic stacks and the anions; the various motions of the methyl groups become affected in different ways, but also the symmetry of the anions is broken and hence the degeneracy of vibrations lifted.

According to our ab-initio quantum-chemical calculations listed in Tables A1 and A2, we expect $\mathrm{C}-\mathrm{C}-\mathrm{H}$ bending modes $\left(\beta-\mathrm{CH}_{3}\right.$ modes $)$ at around 930 and $1020 \mathrm{~cm}^{-1}, \mathrm{H}-\mathrm{C}-\mathrm{H}$ bending modes ( $\alpha-\mathrm{CH}_{3}$ modes) between 1400 and $1440 \mathrm{~cm}^{-1}$ and $\mathrm{C}-\mathrm{H}$ stretching vibrations $\left(\nu-\mathrm{CH}_{3}\right.$ modes) located between 2850 and $2970 \mathrm{~cm}^{-1}$. As illustrated in Figure A2 through Figure A5, most of these vibrations happen within the $a b$-plane, but can also include out-of-plane motions.

For light polarized parallel to the $b^{\prime}$-direction three pronounced modes are detected around 935, 1090 and $1445 \mathrm{~cm}^{-1}$ that not only become stronger upon cooling but also show very distinct temperature dependences best demonstrated in the dielectric losses $\epsilon_{2}(\nu)$ displayed in Figure 13 for (TMTTF $)_{2} \mathrm{AsF}_{6}$ (upper frames) and (TMTTF) ${ }_{2} \mathrm{PF}_{6}$ (lower frames). At room temperature a broad feature is recognized around $938 \mathrm{~cm}^{-1}$ that can be ascribed to the $\mathrm{C}-\mathrm{C}-\mathrm{H}$ bending vibration. These $\beta-\mathrm{CH}_{3}$ modes consist of movements of the hydrogens in opposite directions causing the methyl group to bend; it involves a motion of the outer carbon atoms as depicted in Figures A2(g,h), A3(f), A4(e,f) and A5(c). As the temperature is lowered the band shifts down to $932 \mathrm{~cm}^{-1}$ for $(\mathrm{TMTTF})_{2} \mathrm{AsF}_{6}\left(934 \mathrm{~cm}^{-1}\right.$ for 
$\left.(\mathrm{TMTTF})_{2} \mathrm{PF}_{6}\right)$ and narrows significantly. The observation is confirmed by measurements with $E \| c^{*}$ displayed in Figure 14. We relate the softening of the $923 \mathrm{~cm}^{-1}$ mode to the slowing down of the anion motion, which allows the methyl groups to relax. The most dramatic change occurs when going from $T=20 \mathrm{~K}$ to $13 \mathrm{~K}$; on the high-energy wings two peaks can be identified that split into four when cooled further to $T=6 \mathrm{~K}$. It is tempting to assign these novel sub-features to spin-Peierls transition at $T_{\mathrm{SP}}=13 \mathrm{~K}$; we will come back to this point in Section 6. In the case of (TMTTF $)_{2} \mathrm{PF}_{6}$ the narrowing seems to be more gradual. The mode is also observed for the electric field polarized in $c^{*}$-direction as demonstrated in Figure 14 for the example of (TMTTF) ${ }_{2} \mathrm{PF}_{6}$.

Figure 13. Dielectric losses $\epsilon_{2}(\nu)$ of (TMTTF $)_{2} \mathrm{AsF}_{6}$ and (TMTTF) $)_{2} \mathrm{PF}_{6}$ for light polarized perpendicular to the molecular stacks $\left(E \| b^{\prime}\right)$ in the upper and lower frames, respectively. In the left panels the $\mathrm{C}-\mathrm{C}-\mathrm{H}$ bending vibration is observed around $935 \mathrm{~cm}^{-1}$. The antisymmetric $\nu_{31}\left(b_{1 u}\right)$ mode located around $1090 \mathrm{~cm}^{-1}$ at room temperature splits as $T$ drops below $T_{\mathrm{CO}}=102$ and $67 \mathrm{~K}$, respectively. The right panels show the temperature dependence of the $\mathrm{H}-\mathrm{C}-\mathrm{H}$ bending modes.
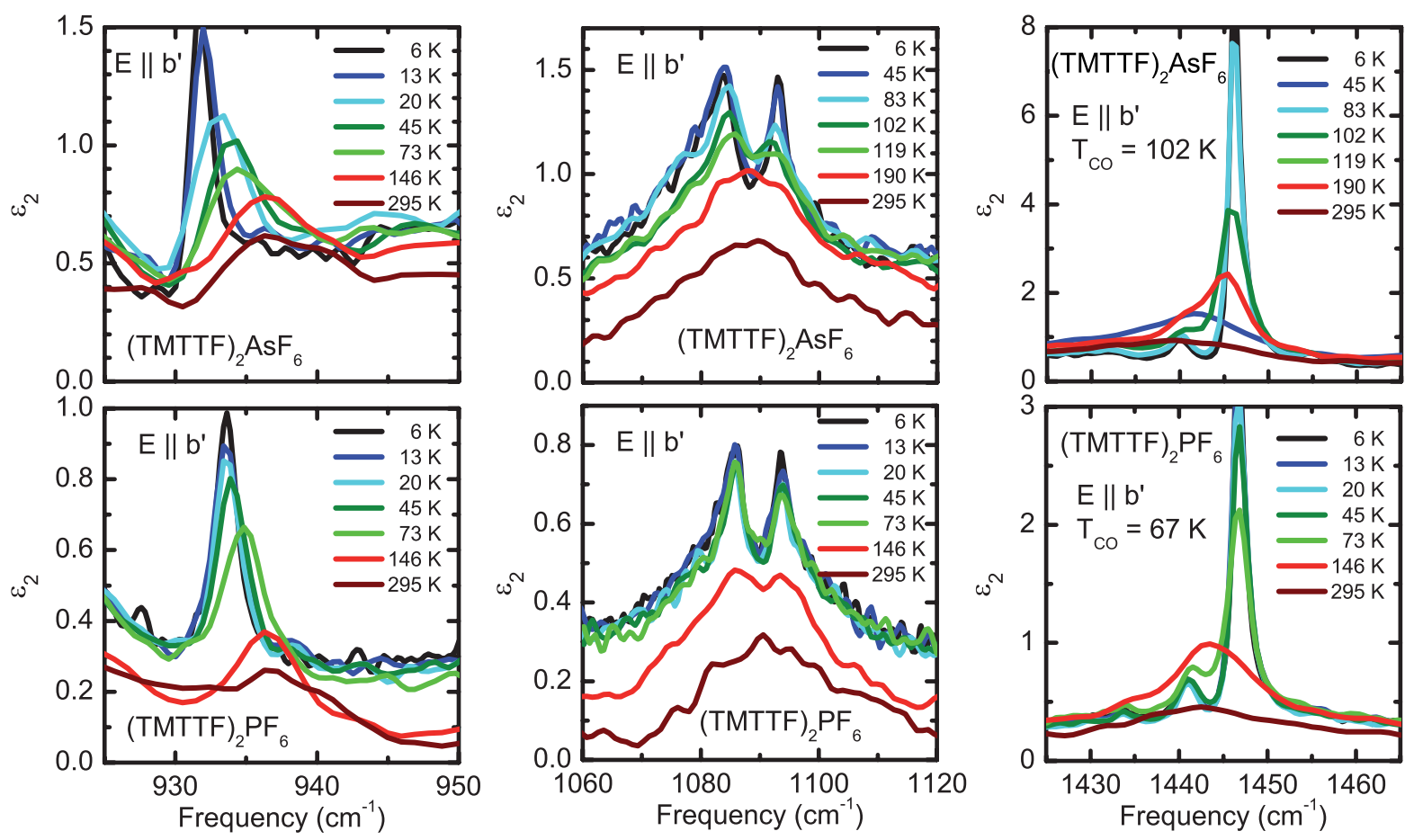

Figure 14. Temperature dependence of the vibrational modes observed in the optical conductivity of (TMTTF) $)_{2} \mathrm{PF}_{6}$ for the polarization direction $E \| c$. The curves are shifted for clarity reasons.
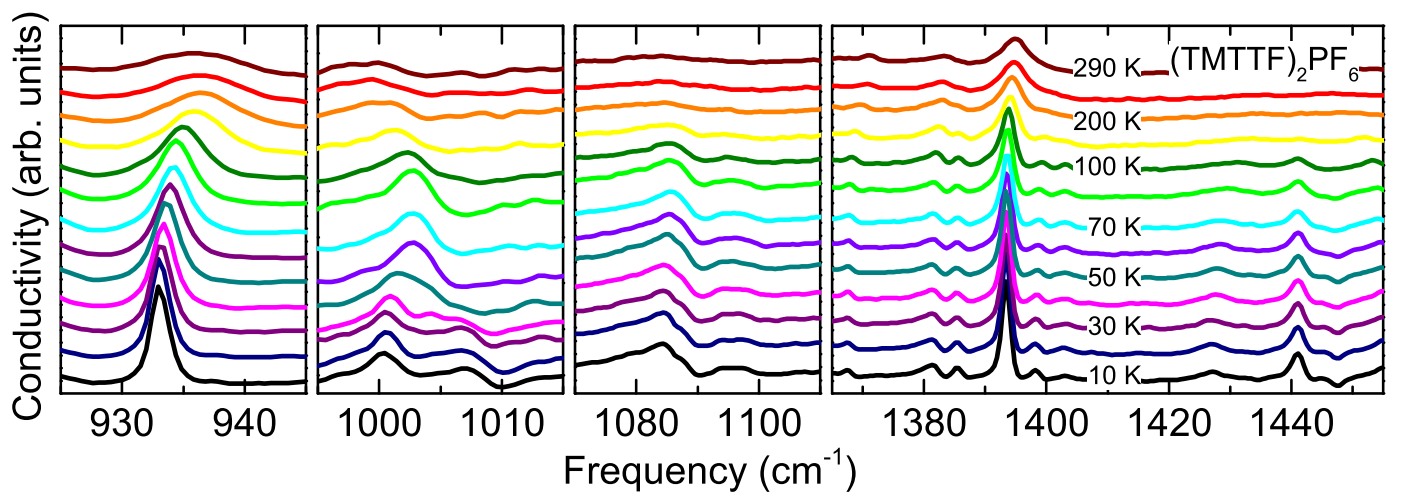
The $1090 \mathrm{~cm}^{-1}$ mode becomes more pronounced upon cooling and splits for low temperatures (middle frame of Figure 13); the behavior is very similar for (TMTTF) ${ }_{2} \mathrm{AsF}_{6}$ and (TMTTF) ${ }_{2} \mathrm{PF}_{6}$. We tentatively assigned the mode to the antisymmetric $\nu_{31}\left(b_{1 u}\right)$ vibration $\left(\beta-\mathrm{CH}_{3}\right)$ which mainly involves the terminal $\mathrm{C}-\mathrm{CH}_{3}$ groups [Figure A3(f)] , very similar to the totally symmetric $\nu_{7}\left(a_{g}\right)$ [Figure A2 $\left.(\mathrm{g})\right]$ that appears in approximately the same frequency range; in the room-temperature Raman spectra (Figure 5) it shows up only as a very small peak. For $E \| c^{*}$ we also observe this mode (Figure 14), but no double structure of similar emphasis. The temperature evolution is somewhat different compared to the modes discussed above in Section 4.2 where mainly the charge disproportionation is probed via the $\mathrm{C}=\mathrm{C}$ bond stretching. There are indications that the $1090 \mathrm{~cm}^{-1}$ mode separates already well above $T_{\mathrm{CO}}$, and once developed, the two peaks basically do not change any more in strength and position. For $E \| c^{*}$ we find only minor indications of this mode with a similar temperature dependence. In the line of previous observations by ${ }^{1} \mathrm{H}$ NMR [92-94], we suggest that the rapid motion of the $\mathrm{CH}_{3}$ groups is locked around $150 \mathrm{~K}$. A clear splitting for $T<T_{\mathrm{CO}}$ can be observed for the $\mathrm{C}-\mathrm{C}-\mathrm{H}$ bending mode located at $1003 \mathrm{~cm}^{-1}$ (Figure 14); at $T=10 \mathrm{~K}$ the peaks are at 1000 and $1007 \mathrm{~cm}^{-1}$ for $(\mathrm{TMTTF})_{2} \mathrm{PF}_{6}$. Note, the presence of this mode as well as its splitting is also observed as an antiresonance in the $E \| a$ spectra of (TMTTF) $)_{2} \mathrm{PF}_{6}$ and (TMTTF) ${ }_{2} \mathrm{AsF}_{6}$ (not shown).

Finally the $\mathrm{H}-\mathrm{C}-\mathrm{H}$ bending vibrations $\left[\alpha-\mathrm{CH}_{3}\right.$, most likely $\nu_{29}\left(b_{1 u}\right)$ or $\left.\nu_{46}\left(b_{2 u}\right)\right]$ are observed at $1440 \mathrm{~cm}^{-1}$; with decreasing temperature they exhibits a hardening up to $1446 \mathrm{~cm}^{-1}$. At low temperatures just above $T_{\mathrm{CO}}$ the band splits and a minor satellite mode is clearly seen at the low-energy side at $1440 \mathrm{~cm}^{-1}$ for both compounds. Basically no change happens for $T<T_{\mathrm{CO}}$ any more (Figure 13). These observations are supported by our Raman experiments presented in Figures 6,7 where three peaks are identified at 1427,1446 and $1459 \mathrm{~cm}^{-1}$. We can safely assign those features to the $\mathrm{H}-\mathrm{C}-\mathrm{H}$ bending modes. For the polarization $E \| c^{*}$ the $1441 \mathrm{~cm}^{-1}$ peak becomes stronger upon cooling with basically no shift in frequency; however, an antiresonance dip is observed at $1447 \mathrm{~cm}^{-1}$ for low temperatures as seem in the right panel of Figure 14. A weak satellite peak seems to develop and shift down to $1427 \mathrm{~cm}^{-1}$ for $T=10 \mathrm{~K}$. We conclude that at the charge-order transition the coupling of the methyl groups to the anions is modified in addition to the pure temperature effect via thermal contraction of the lattice.

A second $\mathrm{H}-\mathrm{C}-\mathrm{H}$ bending mode at $1393 \mathrm{~cm}^{-1}$ is more pronounced; it becomes very narrow (and slightly softer) at low temperatures and it is accompanied by two satellite peaks on each side. Note that for $E \| a$, a feature can be recognized at $1385 \mathrm{~cm}^{-1}$, which is related to the $\alpha-\mathrm{CH}_{3}$ modes $\nu_{30}\left(b_{1 u}\right)$ or $\nu_{47}\left(b_{2 u}\right)$ [Figures A3(e) and A4(d)]. It splits with cooling far above the transition temperatures for all three compounds investigated in our study. From Figure 15 it is obvious that the line shape is asymmetric and significantly broadened at room temperature, indicating that there are different molecular sites present. With increasing anion size, the asymmetry decreases. It is also noteworthy that the strength of the peaks, the onset and the splitting drop with increasing $T_{\mathrm{CO}}$, which can be directly related to the interaction of the anion with the methyl group. These modes involve a particularly strong movement of the $\mathrm{CH}_{3}$ carbon atom in the direction of the anions and thus modifies the cavity size. Its pressure dependence will be examine in more detail in Section 4.5 . 
Figure 15. Temperature dependence of the infrared active $\alpha-\mathrm{CH}_{3}$ mode measured along the $E \| a$ for $(\mathrm{TMTTF})_{2} \mathrm{PF}_{6},(\mathrm{TMTTF})_{2} \mathrm{AsF}_{6}$ and $(\mathrm{TMTTF})_{2} \mathrm{SbF}_{6}$ single crystals. Two distinct peaks develops above $T_{\mathrm{CO}}$ reflecting the interaction of methyl group and the fluorine atoms of the anion.

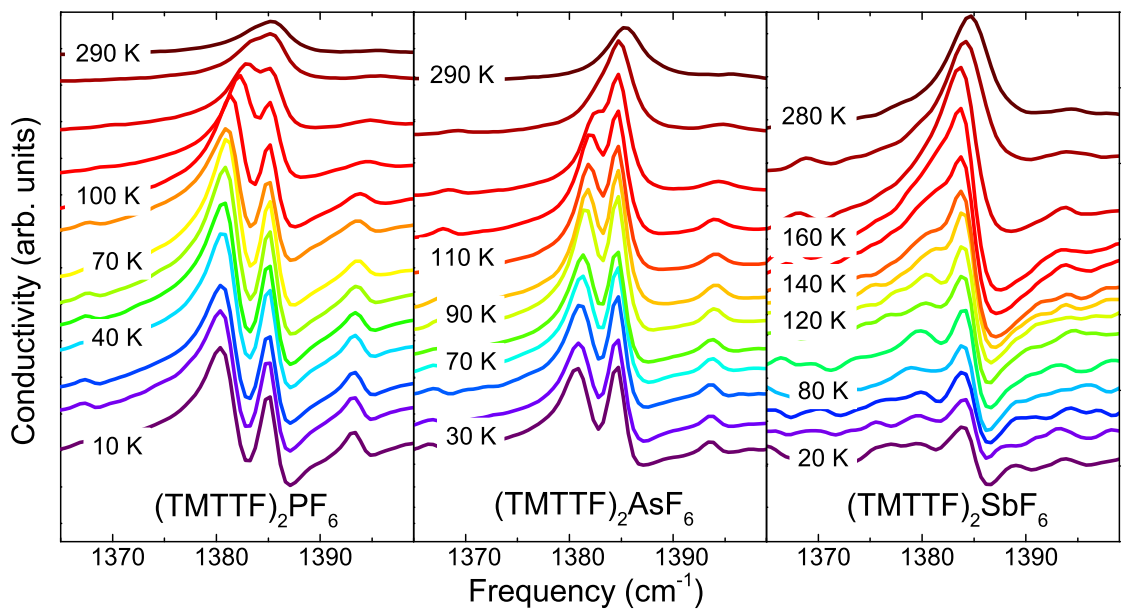

Recently Janokowski et al. [95] observed modifications of the $\mathrm{CH}_{3}$ bands in (o-DMTTF) $)_{2}$ upon cooling that they relate to the hydrogen bonding of halide anions with the methyl groups which influences the $\mathrm{CH}_{3}$ vibrational modes. The splitting of the modes is taken as evidence for breaking the symmetry which yields a small non-equivalence of different hydrogen bonds. However, one has to keep in mind that the cavities formed by the methyl group is cushion-like. This soft and flexible interface allows for a certain amount of disorder [91]. There is no free rotation of the anions at high temperatures [96], but also no complete order at low temperatures.

\subsection{Anions}

In a next step we will look at the anions themselves, i.e., at the molecular vibrations of the pnicogen hexafluorides. Free octahedral ions have $O_{h}$ point group symmetry and 15 vibrational modes that can be represented as [97]

$$
\Gamma=a_{1 g}+e_{g}+2 t_{1 u}+t_{2 g}+t_{2 u} .
$$

Three of these modes $\nu_{1}\left(a_{1 g}\right), \nu_{2}\left(e_{g}\right)$, and $\nu_{5}\left(t_{2 g}\right)$ are Raman active, $\nu_{3}\left(t_{1 u}\right)$ and $\nu_{4}\left(t_{1 u}\right)$ are infrared active; the $t_{2 u}$ mode is inactive. The $\nu_{4}\left(t_{1 u}\right)$ vibration commonly shows up as a very strong feature in the in-plane reflectivity spectra of TMTCF salts [69-72]. As sketched on the right side of Figure 16, for the $\nu_{3}\left(t_{1 u}\right)$ mode the plane of the central pnictide and four fluorine nuclei moves in one direction while the apical fluorine nuclei move the opposite way. In the case of $\nu_{4}\left(t_{1 u}\right)$ the apical nuclei are not involved, and the central pnictide vibrates opposite to the four fluorine nuclei. Both modes are threefold degenerate. Upon cooling, the vibrational motion of the anions slows down and the octahedra eventually lock into a fixed position determined by the cavity that is formed by the methyl groups of the neighboring molecules. A first distortion of this cave occurs from the enormous thermal contraction. In the course of charge ordering, the inversion symmetry is broken and the unbalanced Coulomb attraction exerted by the charge-rich TMTTF molecules causes another distortion of the anions that lifts the degeneracy. It 
turns out that in addition to the cavity of the methyl groups, the interaction of the sulfur atoms to the anions [37,98] is also important, as depicted in Figure 2.

Figure 16. Vibrational spectra of $(\mathrm{TMTTF})_{2} \mathrm{PF}_{6},(\mathrm{TMTTF})_{2} \mathrm{AsF}_{6}$ and $(\mathrm{TMTTF})_{2} \mathrm{SbF}_{6}$ in the range of the anion vibrational mode $\nu_{4}\left(t_{1 u}\right)$ obtained with light polarized perpendicular to the $a b$-plane. The curves for different temperatures are vertically displaced for clarity reasons. As the temperature is reduced below $T_{\mathrm{CO}}$, the modes split by about $7.5 \mathrm{~cm}^{-1}$ due to the distortion of the octahedra. The right side contains a sketch of the two infrared active normal modes of vibration of the octahedral $M \mathrm{~F}_{6}^{-}$ions (where $M=\mathrm{P}, \mathrm{As}$, and $\mathrm{Sb}$ is shown by the red sphere and the fluorine atoms by the blue dots). While $\nu_{3}\left(t_{1 u}\right)$ is around $500 \mathrm{~cm}^{-1}$ and falls outside the mid-infrared range, the $\nu_{4}\left(t_{1 u}\right)$ mode is seen around 650 to $840 \mathrm{~cm}^{-1}$.
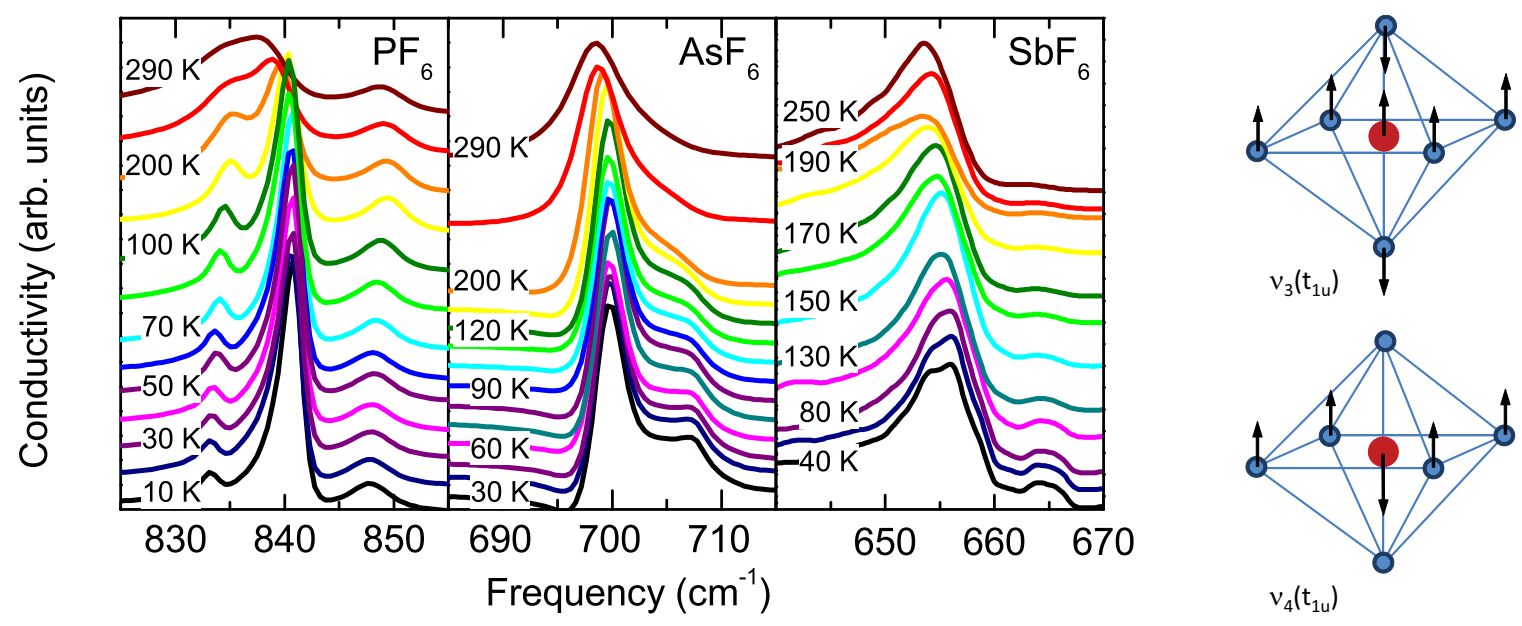

In Figure 16 the conductivity spectra of $(\mathrm{TMTTF})_{2} \mathrm{PF}_{6}$, (TMTTF $)_{2} \mathrm{AsF}_{6}$ and $(\mathrm{TMTTF})_{2} \mathrm{SbF}_{6}$ are presented in the narrow range around the $\nu_{4}\left(t_{1 u}\right)$ mode of the octahedral ions.

At room temperature, the vibrational features are located at $\nu_{4}\left(t_{1 u}\right)=837 \mathrm{~cm}^{-1}$ for $(\mathrm{TMTTF})_{2} \mathrm{PF}_{6}$, at $699 \mathrm{~cm}^{-1}$ for (TMTTF) $)_{2} \mathrm{AsF}_{6}$ and at $654 \mathrm{~cm}^{-1}$ for (TMTTF) $)_{2} \mathrm{SbF}_{6}$. As the temperature approaches $T_{\mathrm{CO}}$ upon cooling, a shoulder starts to develop that finally becomes a satellite peak. The overall behavior is similar for the three compounds, but they differ in important details. In the case of $\mathrm{PF}_{6}^{-}$the mode develops a shoulder at the lower-frequency wing that becomes a separate peak upon cooling. It is not clear whether the separate peak at $848 \mathrm{~cm}^{-1}$ also belong to the anion vibration since it does not change much with temperature. The spectral weight of the satellite peaks is much smaller than the principal mode. The later one shifts to higher frequencies as the temperature is reduced, while the two small modes above and below exhibit a slight softening. The temperature dependence starts at room temperature and gradually develops upon cooling with basically no indications of the charge order transition. Similar observations are made for $(\mathrm{TMTTF})_{2} \mathrm{AsF}_{6}$ and $(\mathrm{TMTTF})_{2} \mathrm{SbF}_{6}$. Since the arsenic atom is heavier than phosphorous, the corresponding mode appears slightly below $700 \mathrm{~cm}^{-1}$ for $(\mathrm{TMTTF})_{2} \mathrm{AsF}_{6}$. Upon cooling a shoulder develops at higher energies $\left(707 \mathrm{~cm}^{-1}\right)$ with an onset well above $T_{\mathrm{CO}}$ and no appreciable shift. This observation implies a distortion that is more related to the lattice modification upon cooling rather than charge order. This is supported by our pressure-dependent measurements discussed in the subsequent Section 4.5 where we follow the mode up to $5.2 \mathrm{GPa}$. The $\mathrm{SbF}_{6}^{-}$anion behaves similarly as $\mathrm{AsF}_{6}^{-}$; a minor mode grows around $664 \mathrm{~cm}^{-1}$ as the temperature is 
reduced, with no obvious modification at $T_{\mathrm{CO}}$. The major peak at $655 \mathrm{~cm}^{-1}$, however, exhibit a slight broadening and eventually splitting for low temperatures.

Unfortunately, our results do not allow us to reliably determine a particular methyl group being responsible for the anion distortion. Due to motion and missing alignment of the anions axes with respect to the crystallographic directions even at low temperatures, we cannot identify a particular contact that cause the distortion. The fact that we mainly observe the anion mode in the polarization $E \| c^{*}$ is caused by the lower out-of-plane electronic contribution.

At elevated temperatures the octahedral anions move rapidly, seen by the broader vibrational features of the anions and methyl groups. From the splitting of the mode at high temperatures we conclude that the distortion is present at all temperatures. Also from NMR experiments it is concluded that at high temperatures the anions are highly disordered and might even rotate, but the rotation of the methyl groups is also important for the coupling [13,92,93,99]. Yu et al. suggested that a suppression of the anion motion effects the charge disproportionation [13]. Rather, our findings point towards a distortion of the anions by lattice contraction and charge order. As pointed out above (Section 4.1, Table 3, Figures 5,2) the charge disproportionation is not reduced when the temperature is lowered and the anions stop rotating. The coupling seems to be strongest for (TMTTF) ${ }_{2} \mathrm{PF}_{6}$ where the splitting below $T_{\mathrm{CO}}$ is most pronounced; for ( $\left.\mathrm{TMTTF}\right)_{2} \mathrm{SbF}_{6}$ the charge order seems to have a minimum influence.

The slowing down of the anions motion softens the methyl group vibrations. Around $T \approx 150 \mathrm{~K}$ the anion position locks in; the $\nu_{31}\left(b_{1 u}\right)$ and other $\beta-\mathrm{CH}_{3}$ modes [involving also the outer carbon atoms of TMTTF and in particular the methyl groups, as depicted in Figure A3(f)] split, indicating two well distinct methyl groups vibrations. For $T<T_{\mathrm{CO}}$ these modes are modified due to the redistribution of charge and coupling to the anions. The lifted degeneracy of anion vibrations indicates the distortion of the octahedra. We conclude that the coupling of the hexafluoride anions to the TMTTF methyl groups forming the cavities (despite the cushion-like softness) changes with temperature but is also affected by the charge order. There mutual interaction modifies the anions as well as the TMTTF molecules.

These findings are corroborated by quantum-chemical calculations based on the low-temperature crystal structure [100] which reveal a deformation of the wave-function of the TMTTF molecules by the anion potential. The distance between TMTTF and $\mathrm{PF}_{6}^{-}$and $\mathrm{SbF}_{6}^{-}$anions decreases as the temperature is reduced; interestingly thermal contraction is not remarkable for the Br-salt. However, the gradual change was seen in the whole temperature, and was continuous through the charge-order temperature. It causes an anomalous temperature behavior of the g-tensor with a continuous rotation of the principal axes around the $a$ axis when $T$ decreases from room temperature to $20 \mathrm{~K}$ [100]. At the charge-order transition temperature $T_{\mathrm{CO}}$ the charge disproportionation on the TMTTF molecules leads to two non-equivalent couplings between the anions and the TMTTF molecules. This broken inversion symmetry of the $(\text { TMTTF })_{2} X$ crystals causes a modification of the spin distribution on the molecules and rotates the g-tensor around the molecular axis. At the domain boundary two inequivalent magnetic sites interact and cause additional contributions to the ESR linewidth [35].

\subsection{Pressure Dependence of Molecular Vibrations}

In order to obtain additional information of the coupling between the anions to the TMTTF molecules and in particular to the $\mathrm{CH}_{3}$ groups, we have performed pressure-dependent optical reflection 
measurements on $(\mathrm{TMTTF})_{2} \mathrm{PF}_{6}$ and $(\mathrm{TMTTF})_{2} \mathrm{AsF}_{6}$. The experiments were performed at room temperature using a diamond anvil cell as discussed in more detail in References [54,55,101]. In Figure 17 we plot the pressure dependence of some vibrational features. It is interesting to note that hydrostatic pressure mainly affects the $a$-direction; X-ray studies under pressure reveal that the $b$ and $c$-axes change only by $4.7 \%$ and $4.0 \%$ with pressure up to $27 \mathrm{kbar}$, while the crystals shrinks by $7.1 \%$ along the $a$-axis [102]. The cavities containing the anions get distorted.

Figure 17. Pressure dependence of the optical conductivity of (TMTTF $)_{2} \mathrm{AsF}_{6}$ measured at room temperature for $E \| a$. The spectral range of those features is enlarged that are related to vibrations of the TMTTF methyl groups.
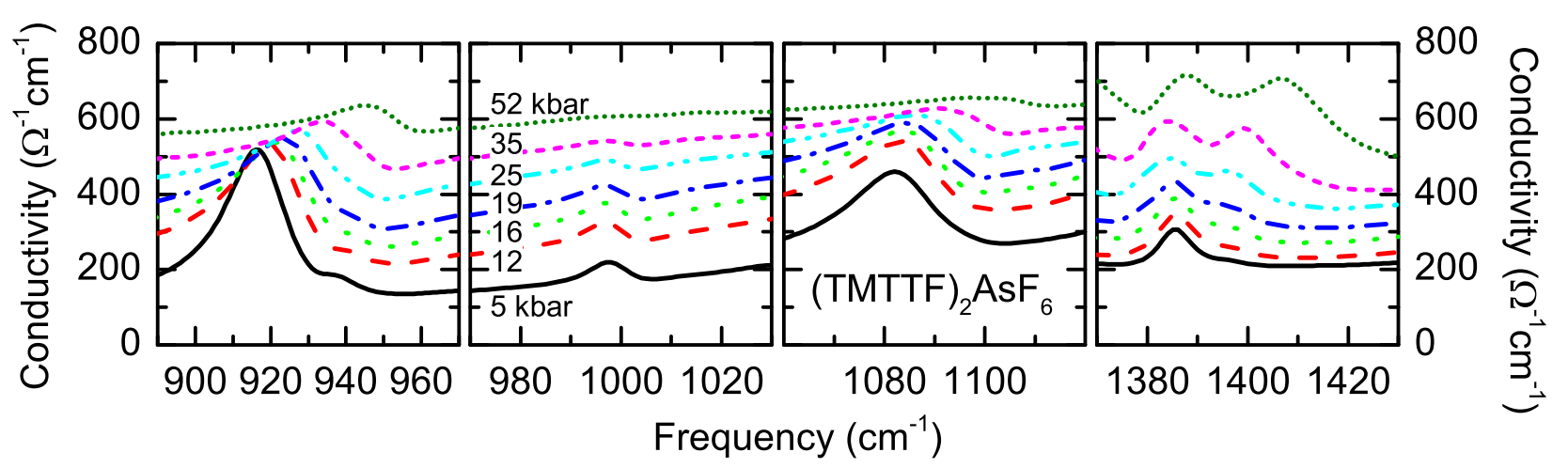

The pressure dependence of the optical conductivity is shown in Figure 17 for selected frequency ranges. Most pronounced is the shift of the $\mathrm{C}-\mathrm{C}-\mathrm{H}$ bending mode at $915 \mathrm{~cm}^{-1}$ that moves by more than $25 \mathrm{~cm}^{-1}$ as the pressure increases to $50 \mathrm{kbar}$, indicating a significant hindering of the vibration. The mode at $1082 \mathrm{~cm}^{-1}$, for which we have suggested the assignment $\nu_{31}\left(b_{1 u}\right)$ above, exhibits a very similar pressure dependence. On the contrary, the peak $998 \mathrm{~cm}^{-1}$ basically remains unchanged in frequency and just becomes smeared out until it has disappeared. It is interesting to note that for moderate pressure, the peak shifts to lower energies, in contrast to most other modes. The most pronounced modification upon applying high pressure is found for the $\nu_{47}\left(b_{2 u}\right)=1385.5 \mathrm{~cm}^{-1}$ mode, which involves the $\mathrm{C}-\mathrm{H}$ bending vibrations of the methyl groups [symmetric $\alpha-\mathrm{CH}_{3}$ vibration, sketched in Figure A4(d)]. The mode develops a shoulder at $1397 \mathrm{~cm}^{-1}$ that becomes a well developed double peak when going up to 52 kbar. From the crystal structure displayed in Figure 2a we see that within the $b^{\prime} c^{*}$-plane two sorts of methyl groups can be distinguished, one more directed along the $c$-axis, the other more along the b-direction. The application of pressure strongly affects the coupling of the $\mathrm{CH}_{3}$ groups, but differently for both sorts. Methyl groups form a soft cushion surrounding the anions that is squeezed upon pressure, getting stiffer and denser. However, not all of them are affected equally, leading to a broadening and splitting of the modes.

The pressure dependence of two other peaks in the (TMTTF) $)_{2} \mathrm{AsF}_{6}$ spectra are worth mentioning. First the fingerprint of the $\nu_{3}\left(a_{g}\right)$ mode close to $1600 \mathrm{~cm}^{-1}$ for which the temperature dependence was already discussed in Section 4.2. Its broadening with pressure with some indication of splitting may be seen as evidence that the two molecules per unit cell are influenced differently.

The most interesting behavior is presented in the left frame of Figure 18, where the anion vibrational mode is seen. Upon application of high hydrostatic pressure, the mode shifts to higher frequencies, 
becomes broad and finally exhibits two satellite peaks left and right to the main maximum at $717 \mathrm{~cm}^{-1}$, separated by approximately $\pm 8 \mathrm{~cm}^{-1}$. The threefold degeneracy of the $\nu_{4}\left(t_{1 u}\right)$ mode is lifted as the hexafluoride anion becomes distorted at high pressure. This implies that the motion of the anions is stopped by the methyl groups and eventually the strong pressure completely breaks the degeneracy.

Figure 18. Pressure dependence of the optical conductivity of (TMTTF) $)_{2} \mathrm{AsF}_{6}$ measured at room temperature for $E \| a$. The $700 \mathrm{~cm}^{-1}$ mode is related to the $\mathrm{AsF}_{6}^{-}$vibration, while the $1600 \mathrm{~cm}^{-1}$ peak is related to the asymmetric $\mathrm{C}=\mathrm{C}$ stretching vibration of the TMTTF molecule.
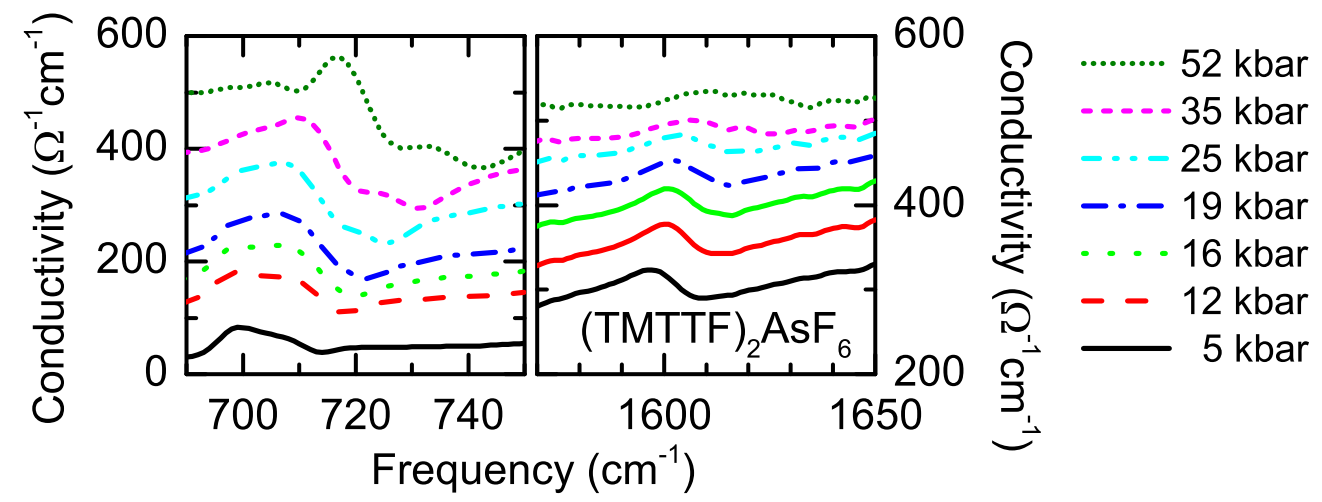

\subsection{Anharmonicity in One-Dimensional Organic Conductors}

The Raman spectra illustrated in Figure 5 reveal modes that originate from combinations and overtones of fundamental modes. These modes can be relocated in the infrared spectra along the stacking direction exhibiting a splitting below $T_{\mathrm{CO}}$ and delivering further information about charge ordering and the electronic potential.

In Figure 19 we plot the temperature dependence of a mode at $805 \mathrm{~cm}^{-1}$ that is a combination of the emv-coupled $\nu_{11}\left(a_{g}\right)$ and $\nu_{10}\left(a_{g}\right)$ modes. It can be observed as a peak or dip depending on electronic background ( $c f$. Section 2). The occurrence of the higher-frequency peaks throughout the entire temperature range indicates that here we deal with an anharmonic electronic potential of the $\mathrm{TMTTF}^{+0.5}$ molecule. Both modes are members of the same symmetry species $a_{g}$, consequential the combination $\left(\nu_{11}+\nu_{10}\right)$ mode possess the same symmetry as the fundamental ones; i.e., it is Raman active and simultaneously becomes infrared-activated via emv-coupling. This symmetry consideration holds for all modes discussed in this Section.

The $\nu_{11}$ and the $\nu_{10}$ modes involve the stretching of the $\mathrm{C}-\mathrm{S}$ bond and the bending of the $\mathrm{C}-\mathrm{CH}_{3}$ bond; both modes are quite sensitive to changes of the ionicity. Beside the $\nu_{4}$ mode, the $\nu_{10}$ has the highest coupling constant [75] which are listed in Table 4. In the Raman spectra displayed in Figure 5b, the $\left(\nu_{11}+\nu_{10}\right)$ mode is also present as a weak peak at $800 \mathrm{~cm}^{-1}$. According to Meneghetti et al. [59] the maximum value of the splitting between a neutral and a positive charged molecule is $\Delta \nu=50 \mathrm{~cm}^{-1}$ resulting for $(\mathrm{TMTTF})_{2} \mathrm{PF}_{6}$ in $2 \delta=0.14 e$ and for $(\mathrm{TMTTF})_{2} \mathrm{AsF}_{6}$ in $2 \delta=0.20 e$ (in analogy to Equations (1) and (2)). 
Figure 19. Infrared spectra of $(\mathrm{TMTTF})_{2} \mathrm{PF}_{6}$ and $(\mathrm{TMTTF})_{2} \mathrm{AsF}_{6}$ as function of temperature measured along the stacking direction $(E \| a)$. For the two compounds the clear splitting of the antiresonance combination mode $\nu_{11}\left(a_{g}\right)+\nu_{10}\left(a_{g}\right)$ is visible. In the case of (TMTTF $)_{2} \mathrm{SbF}_{6}$ the data were too noisy to present here. The spectra are shifted with a constant value for clarification. In the lower panel the same mode is presented, for the example of (TMTTF $)_{2} \mathrm{PF}_{6}$, but measured for $E$ polarized perpendicular to the $a b$-plane. It is interesting to note that the two peaks observed for $E \| c^{*}$ spectra become dips in the $E \| a$ polarization due to the emv interaction with the electronic background with a clear Fano shape at elevated temperatures. In the case of (TMTTF $)_{2} \mathrm{AsF}_{6}$ peaks are observed also for the light polarized parallel to the stacks.

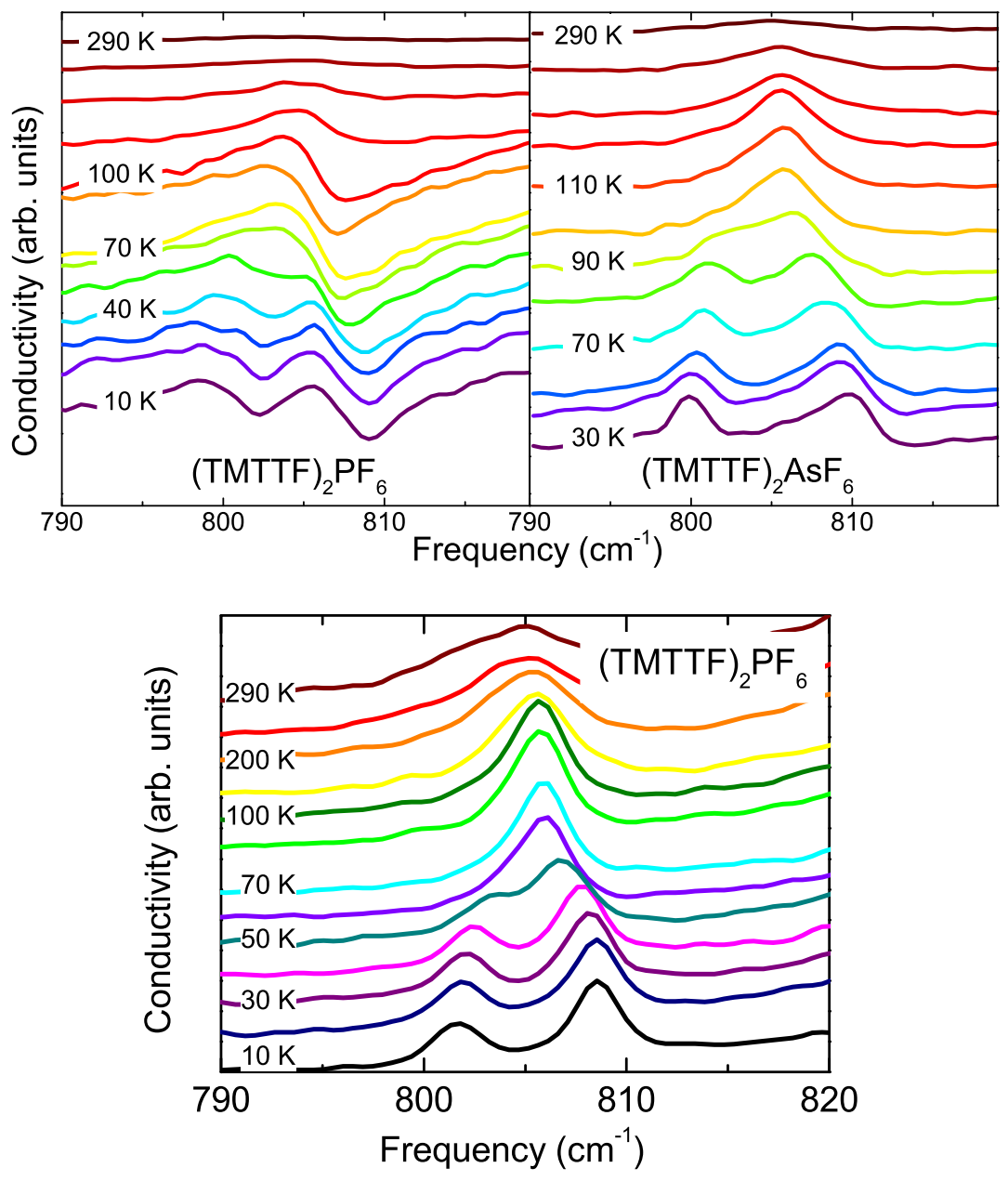

Table 4. The emv-coupling constants for $a_{g}$ modes of the TMTTF molecule calculated by Pedron et al. [75].

\begin{tabular}{lrrrrrrrr}
\hline Mode & $\boldsymbol{\nu}_{\mathbf{3}}$ & \multicolumn{1}{c}{$\boldsymbol{\nu}_{\mathbf{4}}$} & $\boldsymbol{\nu}_{\mathbf{7}}$ & $\boldsymbol{\nu}_{\mathbf{8}}$ & $\boldsymbol{\nu}_{\mathbf{9}}$ & $\boldsymbol{\nu}_{\mathbf{1 0}}$ & $\boldsymbol{\nu}_{\mathbf{1 1}}$ & $\boldsymbol{\nu}_{\mathbf{1 2}}$ \\
\hline Frequency $\left(\mathrm{cm}^{-1}\right)$ & 1,639 & 1,538 & 1,092 & 934 & 560 & 494 & 277 & 217 \\
$g_{i}\left(\mathrm{~cm}^{-1}\right)$ & 242 & 968 & 242 & 242 & 161 & 484 & 81 & 81 \\
\hline
\end{tabular}

A similar observation can be made for the first overtone of the $\nu_{10}$ mode which is also visible in all Raman spectra (Figure 5) at $1000 \mathrm{~cm}^{-1}$ where it appears as an asymmetric broad peak. In the 
infrared spectra (Figure 20), the single asymmetric peak at room temperature evolutes into two dips after cooling below $T_{\mathrm{CO}}=67 \mathrm{~K}$ for $(\mathrm{TMTTF})_{2} \mathrm{PF}_{6}$, for instance. In the case of the two other compounds, $(\mathrm{TMTTF})_{2} \mathrm{AsF}_{6}$ and $(\mathrm{TMTTF})_{2} \mathrm{SbF}_{6}$, we can actually identify a doubling of the splitting at very low temperatures, indicating that four different sites exists in the unit cell, although the other modes do not support this conclusion. The maximum amplitude of the mode splitting is $\Delta \nu=48 \mathrm{~cm}^{-1}$ at the lowest temperature yielding a charge imbalance of $0.14 e$ for $(\mathrm{TMTTF})_{2} \mathrm{PF}_{6}, 0.19 e$ for $(\mathrm{TMTTF})_{2} \mathrm{AsF}_{6}$ and $0.26 e$ for $(\mathrm{TMTTF})_{2} \mathrm{SbF}_{6}$, which is also in good agreement with the values listed in Table 3. In Figure 21 we plot the spectra of the compounds, (TMTTF $)_{2} \mathrm{PF}_{6}$ and (TMTTF) $)_{2} \mathrm{AsF}_{6}$, measured for the polarization $E \| a$. For (TMTTF) ${ }_{2} \mathrm{SbF}_{6}$ the spectra were too noisy to identify any vibrational features in this spectral range. At higher frequency a vibrational feature is observed at $1805 \mathrm{~cm}^{-1}$ that splits very similarly to the emv-coupled $\nu_{3}\left(a_{g}\right)$ mode. At this point, no fundamental vibrational mode should exist, but it can be assigned to the combination mode of $\nu_{4}+\nu_{11}$. Depending on the electronic background, emv-coupled modes can show up either as dips or as peaks in the conductivity spectra ( $c f$. Section 2 ).

Figure 20. Temperature-dependent infrared spectra for the $2 \nu_{10}$ mode obtained for (TMTTF $)_{2} \mathrm{PF}_{6}$, (TMTTF $)_{2} \mathrm{AsF}_{6}$ and (TMTTF) $)_{2} \mathrm{SbF}_{6}$ with $E \| a$. The arrows indicate the evolution of the resonance with decreasing temperature.

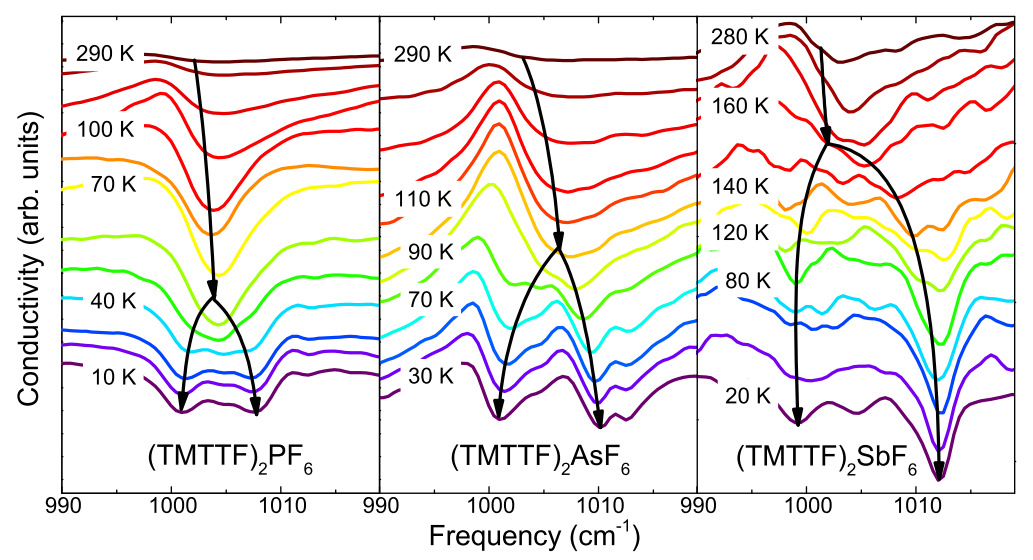

Figure 21. Mid-infrared conductivity $\sigma_{1}(\nu)$ of (TMTTF $)_{2} \mathrm{PF}_{6}$ and (TMTTF $)_{2} \mathrm{AsF}_{6}$ for light polarized parallel to the molecular stacks $(E \| a)$ in the vicinity of the emv-coupled totally symmetric intramolecular $\nu_{3}\left(a_{g}\right)$ mode for temperatures above and below $T_{\mathrm{CO}}$. The curves are displaced by a constant factor to avoid overlap. The arrows indicate the positions of the antiresonance dips. In the case of (TMTTF) $)_{2} \mathrm{SbF}_{6}$ the spectra were too noisy to identify any clear feature.

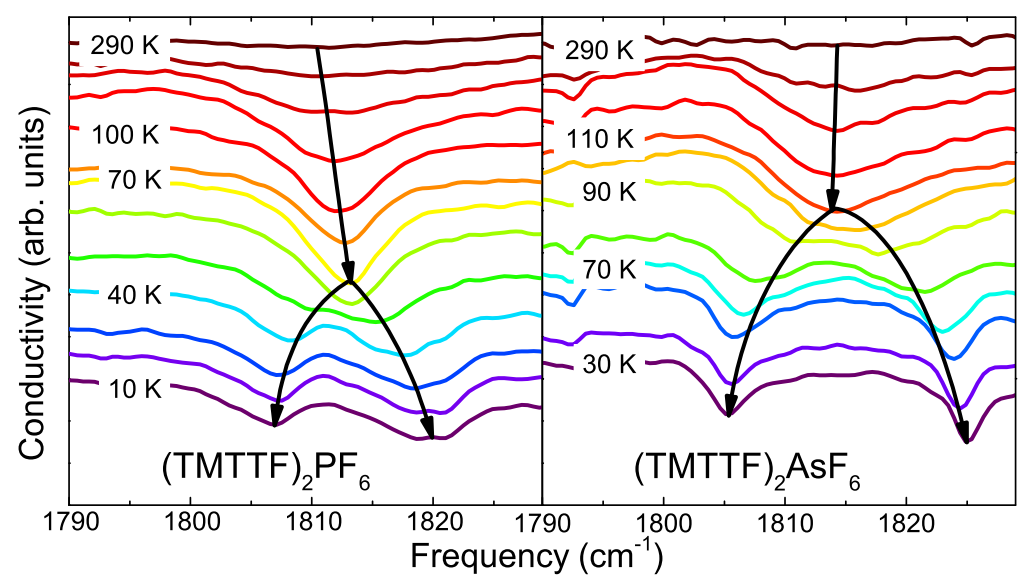


Beside the mentioned and charge sensitive modes, two broad features can be noticed in the lower and upper panels of Figure 22 in the frequency ranges between $1800 \mathrm{~cm}^{-1}$ and $2000 \mathrm{~cm}^{-1}$ and between $2600 \mathrm{~cm}^{-1}$ and $2800 \mathrm{~cm}^{-1}$. The feature located at around $1900 \mathrm{~cm}^{-1}$ is basically not apparent at room temperature, but gets really pronounced at the charge order transition. It can be ascribed to the combination mode of the two strongest emv-coupled $\nu_{4}$ and $\nu_{10}$ modes. The intensity clearly scales with the charge disproportionation increasing with the anion size.

Figure 22. Temperature dependence of the infrared spectra of $(\mathrm{TMTTF})_{2} \mathrm{PF}_{6}$, $(\mathrm{TMTTF})_{2} \mathrm{AsF}_{6}$ and $(\mathrm{TMTTF})_{2} \mathrm{SbF}_{6}$ obtained from reflection measurements along the stacking direction $(E \| a)$. In the upper and lower panels, two distinct features are highlighted marking the combination mode of $\nu_{4}+\nu_{10}$ and the first overtone of $2 \nu_{4}$, respectively.
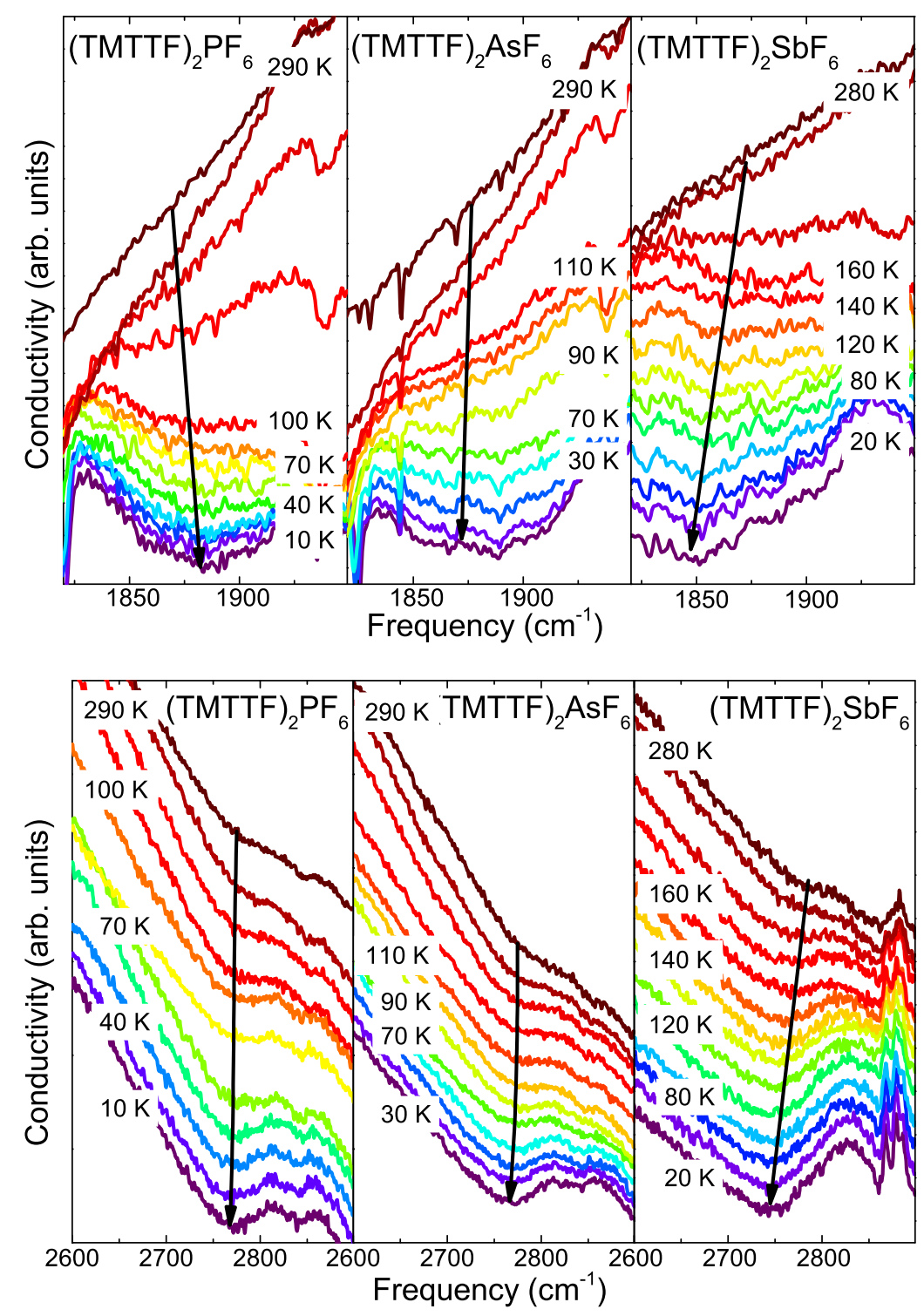

The highest anomaly is located at $2750 \mathrm{~cm}^{-1}$ and cannot be related to the aforementioned methyl vibrations which are found between 2850 and $3000 \mathrm{~cm}^{-1}$. The feature is already present at room temperature where it manifests as a weak dip that is strongest for (TMTTF) $)_{2} \mathrm{SbF}_{6}$. Its strength increases 
with the anion size from $(\mathrm{TMTTF})_{2} \mathrm{PF}_{6}$ to $(\mathrm{TMTTF})_{2} \mathrm{SbF}_{6}$. At $T_{\mathrm{CO}}$ the mode gains intensity. According to the theoretical calculations and the infrared and Raman measurements by Meneghetti et al. [59], no fundamental mode can be assigned to the resonance.

Hence it can be related to the overtone of $\nu_{4}\left(a_{g}\right)$ being strongly coupled to the transition band. One can exclude that the resonance arise from the overlap of different transition bands. This dip-like optical structure also appears in two-dimensional organic conductors $[103,104]$ as well in other one-dimensional systems $[95,106]$ with and without a charge-order state.

In the literature of organic conductors not much attention has been devoted to these features and anharmonicity in general. Very recently Yamamoto et al. $[103,105]$ considered these anomalies and provided a first explanation of the spectral structure by applying a model of a diatomic dimer systems including emv-coupling. It reveals that the overtone of strongly coupled modes get activated by charge disproportionation. Taking into account higher order terms of the vibronic perturbation, they receive two terms describing the anharmonicity effect. One term is connected to the charge-transfer matrix which is present in all systems leading to a non-zero activation of the combination and overtones in any system, whereas the second term includes the charge disproportionation strongly contributing to the activation of higher modes. Both terms compete with each other, however, with increasing charge disproportionation the influence of the charge-transfer term diminishes.

If we consider now our one-dimensional system, we have a finite contribution of the charge transfer that leads to the activation of the higher modes at all temperatures, which is the main reason why we see the effect in all three compounds. But as soon as the systems enters the charge-order phase, the charge-separation term takes over. For (TMTTF $)_{2} \mathrm{PF}_{6}$, the system has a low charge imbalance, thus the contribution of the second term is marginal; therefore the strength of the features is very similar above and below $T_{\mathrm{CO}}$. But the situation changes if we go to (TMTTF) ${ }_{2} \mathrm{SbF}_{6}$ where the charge disproportionation is $2 \delta=0.3 e$; here the second term dominates and a huge change sets in below $T_{\mathrm{CO}}$. According to Yamamoto et al. [103] the maximum influence of the second term is at $0.85 e$, implying that for such kind of charge-ordered system the effect must be even stronger. To support this interpretation, it would be desirable to conduct an infrared study on the new synthesized organic salt (TMTTF) ${ }_{2} \mathrm{TaF}_{6}$ [107] where the transition temperature is at $T_{\mathrm{CO}}=175 \mathrm{~K}$, connected with a larger charge imbalance. Here we could show that from the overtones and the combination modes we can gain further insight into the electronic interaction; in general they can be used to observe the onset of the charge ordering.

\section{Low-Energy Spectroscopy: Lattice Phonons}

Our investigations of the intramolecular vibrations presented above provide clear evidence that charge disproportionation between the organic molecules occurs in various (TMTTF) ${ }_{2} X$ salts, as summarized in Table 3. We also provided a complex picture of the coupling to the anions and the important influence of the methyl groups forming a soft cavity around the octahedra. The next question now is the charge-order pattern, i.e., the lattice symmetry of the charge distribution. To that end we extended our Raman experiments down to low-energy $\left(20-200 \mathrm{~cm}^{-1}\right)$ and supplemented the findings with far-infrared reflection data, reaching down to $40 \mathrm{~cm}^{-1}$. 


\subsection{Symmetry Analysis}

First let us analyze the symmetry of the unit cell and vibrational modes of the (TMTTF) $)_{2} \mathrm{X}$ salts crystallized in the triclinic $P \overline{1}$ space. Following Krauzman et al. [108] and based on careful structure analysis, the stack dimerization can be neglected and a pseudo-monoclinic unit cell approximated with $C_{2 h}$ symmetry. In the spectral region below $200 \mathrm{~cm}^{-1}$ at most 15 lattice modes are expected: 6 due to the rigid translations and rotations of the anions and 9 due to the (TMTTF) $)_{2}$ dimer degrees of freedom. In a first approximation we can describe the dimer degrees of freedom as the three anti-phase rigid translations $\left(T_{a}, T_{b}, T_{c}\right)$, three in-phase and three anti-phase librations $\left(3 R^{+}, 3 R^{-}\right)$of the two TMTTF moieties. Within the $C_{2 h}$ symmetry six dimer modes are Raman active $\left(A_{g}, B_{g}\right)$ and three are infrared active $\left(A_{u}, B_{u}\right)$ as schematically represented in Table 5 , together with their corresponding polarization dependence. The anion translations are also infrared active and have $A_{u}$ symmetry (translation along $a$-axis), and $B_{u}$ symmetry (translations perpendicular to the $a$ axis), while the three anion rotations are Raman active with one $A_{g}$ and two $B_{g}$ symmetry modes. We note that rotations and libration modes owe their Raman intensity uniquely from the rigid rotations of the molecular dielectric tensor; therefore they usually have very weak intensity and can hardly be observed. This is especially true for octahedral anions where the dielectric tensor has more or less a spherical symmetry.

Table 5. Analysis of the (TMTTF) $)_{2}$ lattice modes using the approximated $C_{2 h}$ unit cell.

\begin{tabular}{ccc}
\hline $\mathbf{C}_{\mathbf{2} \boldsymbol{h}}$ & Lattice Modes & Polarization \\
Symmetry & & \\
\hline $\mathbf{A}_{\mathbf{g}}$ & $T_{b}, T_{c}, R_{a}^{+}$ & $a a, b^{\prime} b^{\prime}, c c, b^{\prime} c$ \\
$\mathbf{B}_{\mathbf{g}}$ & $T_{a}, R_{b}^{+}, R_{c}^{+}$ & $a b^{\prime}, a c$ \\
$\mathbf{A}_{\mathbf{u}}$ & $R_{a}^{-}$ & $a$ \\
$\mathbf{B}_{\mathbf{u}}$ & $R_{b}^{-}, R_{c}^{-}$ & $b^{\prime}, c$ \\
\hline
\end{tabular}

Summarizing, the analysis reveals that two dimer translation modes $\left(T_{b}\right.$ and $\left.T_{c}\right)$ are expected in the $A_{g}$ Raman spectra, which probe the diagonal components of the polarizations tensor $\left(a a, b^{\prime} b^{\prime}\right.$ and $c c$ polarization); and one dimer translation $\left(T_{a}\right)$ in the $B_{g}$ spectrum characterized by off-diagonal elements of the tensor ( $a b^{\prime}$ and $a c$ crossed polarization). Instead, anion translations can be detected in the infrared spectra, in particular the mode with $A_{u}$ symmetry in the spectra polarized along the $a$-axis, and the two modes with $B_{u}$ symmetry in the spectra polarized along the $b^{\prime}$-direction.

\subsection{Far-Infrared Spectra}

In Figure 23 the far-infrared reflectivity spectra of $(\mathrm{TMTTF})_{2} \mathrm{AsF}_{6}$ and $(\mathrm{TMTTF})_{2} \mathrm{PF}_{6}$ are plotted for two different polarizations: $E \| a$ and $E \| b^{\prime}$. When the temperature is reduced below $T_{\mathrm{CO}}=102$, and $67 \mathrm{~K}$, respectively, three vibrations become infrared active due the charge disproportionation between charge rich $\left(\rho_{0}+\delta\right)$ and charge poor $\left(\rho_{0}-\delta\right)$ molecules. In a first attempt these modes are assigned to the three anti-phase translation modes in the $a, b$ and $c$-direction. As inversion symmetry is lost in the charge-ordered state, a permanent dipole moment develops (electronic ferroelectricity) with a charge 
distribution $\cdots+-+-+-$. For $(\mathrm{TMTTF})_{2} \mathrm{AsF}_{6}$ the strong $T_{a}\left(B_{g}\right.$ symmetry) mode develops at $85 \mathrm{~cm}^{-1}$ when probed along the stacking direction; in the perpendicular polarization, two vibrational features are observed: at $54 \mathrm{~cm}^{-1}\left[T_{b}\left(A_{g}\right)\right]$ and $66 \mathrm{~cm}^{-1}\left[T_{c}\left(A_{g}\right)\right]$. In the (TMTTF) $)_{2} \mathrm{PF}_{6}$ compound we find two modes for $E \| a$ at 68 and $83 \mathrm{~cm}^{-1}\left[T_{a}\left(B_{g}\right)\right]$, and one at $54 \mathrm{~cm}^{-1}\left[T_{b}\left(A_{g}\right)\right]$ in the perpendicular direction. The comparison with our Raman spectra will support and advance the assignment.

The rather strong band observed in (TMTTF) ${ }_{2} \mathrm{PF}_{6}$ at $68 \mathrm{~cm}^{-1}$ cannot be satisfactorily assigned at this point. We suggest that the $68 \mathrm{~cm}^{-1}$ band is probably the internal vibration, known as "boat-mode" [109]. This low-frequency mode is infrared active for $E \| a$ but also has a Raman counterpart, as demonstrated in Figure 24. It strongly couples to the charge-order transition because it describes the distortion mode which links neutral TMTTF (boat conformation) and $\mathrm{TMTTF}^{+}$(flat conformation). Thus this modes can modulate the charge transfer between molecules in weakly charge-ordered system, such $(\mathrm{TMTTF})_{2} \mathrm{PF}_{6}$. In strong charge-ordered system, such as (TMTTF $)_{2} \mathrm{AsF}_{6}$, it is not observed, probably hindered by the strong band at $85 \mathrm{~cm}^{-1}$. We may want to point out the $75 \mathrm{~cm}^{-1}$ feature also observed in (TMTTF $)_{2} \mathrm{AsF}_{6}$ as a very weak shoulder that might the analogue to the $68 \mathrm{~cm}^{-1}$ in (TMTTF) ${ }_{2} \mathrm{PF}_{6}[110]$. In (TMTTF $)_{2} \mathrm{AsF}_{6}$ the charge disproportionation is more stable and the mode is not strong enough to move the charge between molecules and develop an oscillating dipole moment. In Section 6 we suggest a relation to the spin-Peierls transition since it splits for $T<T_{\mathrm{SP}}$ as demonstrated in Figure 25.

Figure 23. Far-infrared reflectivity spectra of (TMTTF $)_{2} \mathrm{AsF}_{6}$ (upper panels) and $(\mathrm{TMTTF})_{2} \mathrm{PF}_{6}$ salts (lower panels) measured at different temperatures with the polarization parallel ( $E \| a$, left panels) and perpendicular ( $E \| b^{\prime}$, right panels) to the stacks. As $T$ drops below $T_{\mathrm{CO}}$ strong vibrational features develop at $54,66,75$ and $85 \mathrm{~cm}^{-1}$ in the case of $(\mathrm{TMTTF})_{2} \mathrm{AsF}_{6}$, and at 54, 68, and $83 \mathrm{~cm}^{-1}$ for (TMTTF) ${ }_{2} \mathrm{PF}_{6}$. The excitations are mainly related to the translational lattice modes $T_{a}, T_{b}$ and $T_{c}$ sketched on the right side for the example of a TMTTF dimer.
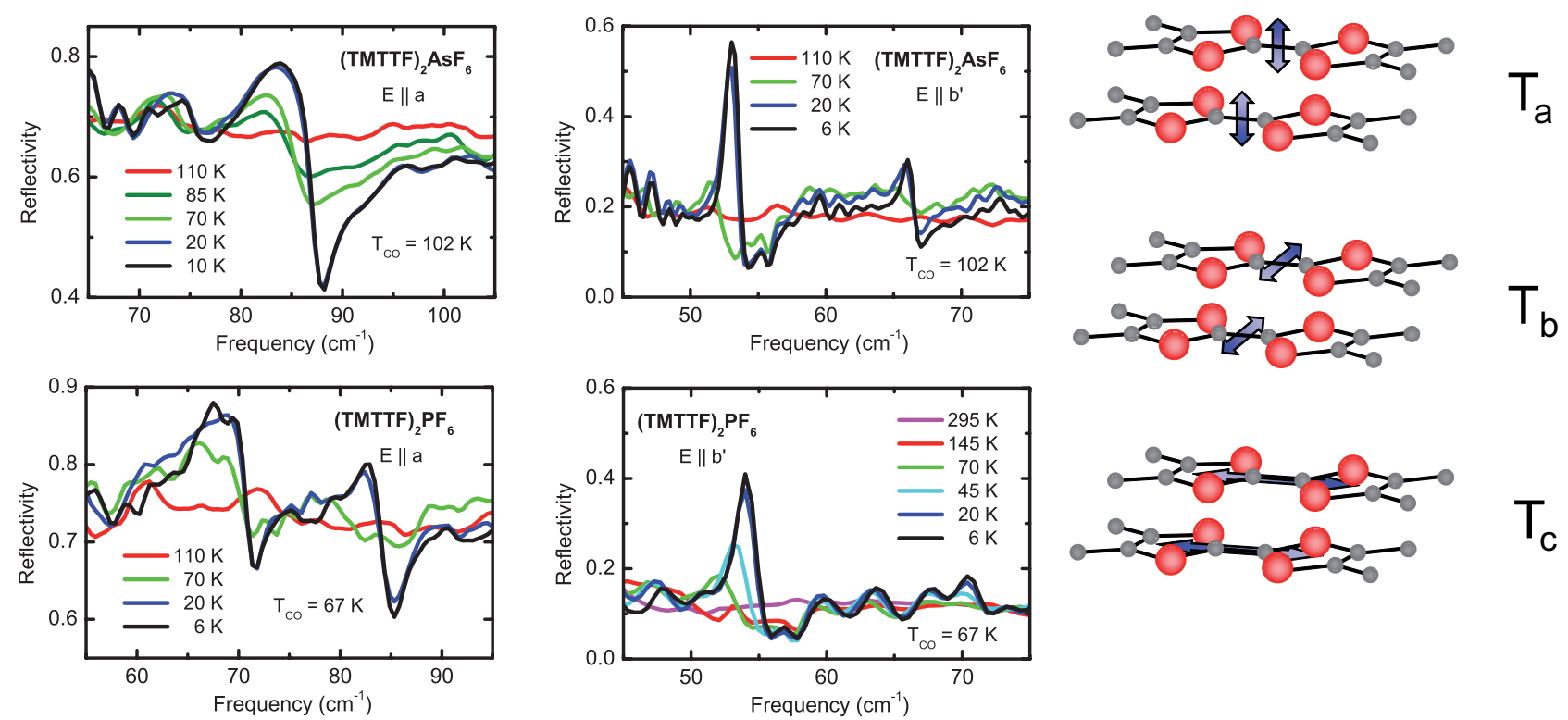
Figure 24. Raman spectra of (TMTTF) ${ }_{2} \mathrm{PF}_{6}$ compared to (TMTTF) $)_{2} \mathrm{AsF}_{6}$ measured with $\lambda=647 \mathrm{~nm}$ laser line in the $\left(b^{\prime}, b^{\prime}\right)$-polarization at $T=15 \mathrm{~K}$. For the assignment of the modes, confer with Table 7 .

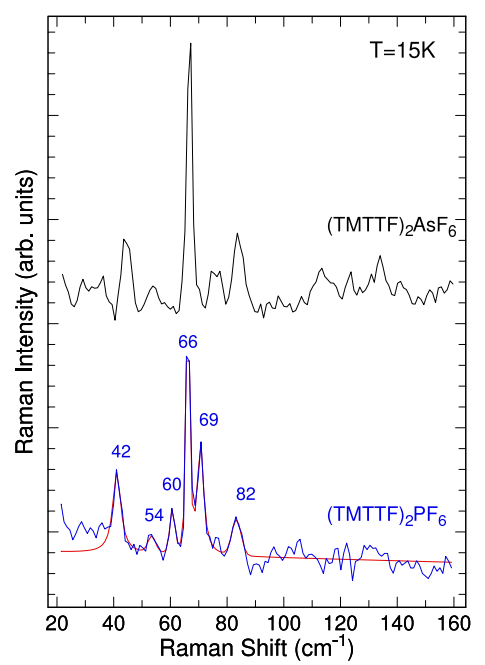

Figure 25. The absorption spectra exhibit the temperature-development of the $75 \mathrm{~cm}^{-1}$ lattice mode in (TMTTF $)_{2} \mathrm{AsF}_{6}$ on the left side. In the right panel, a weak band develops at $461 \mathrm{~cm}^{-1}$ that might be a satellite of the double structure seen at 452 and $457 \mathrm{~cm}^{-1}$.

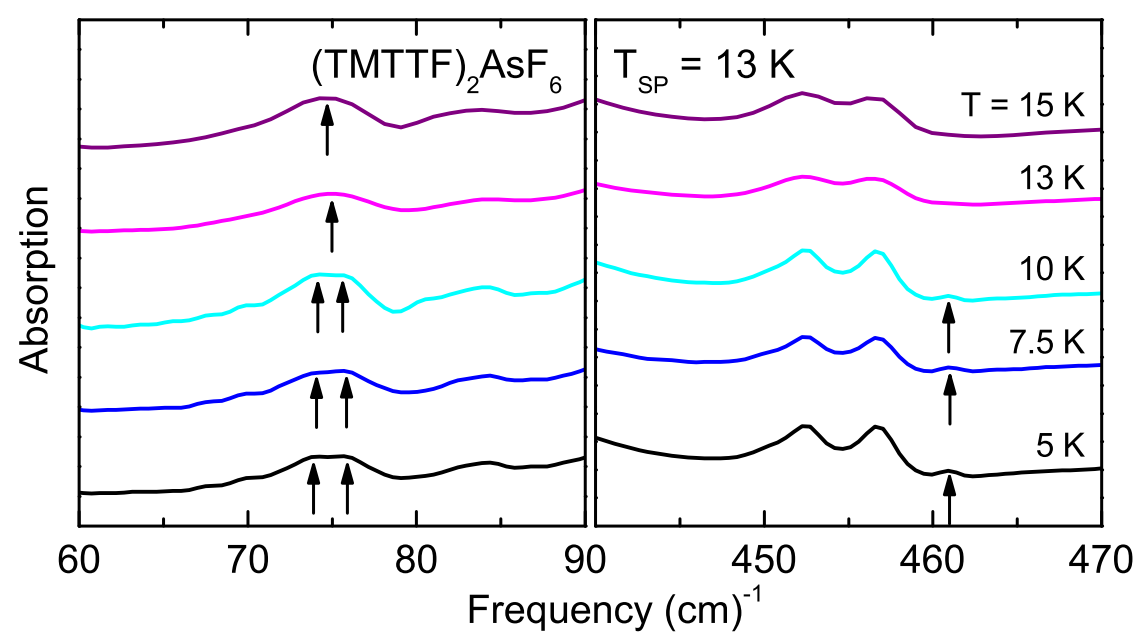

An interesting development can be identified around $110 \mathrm{~cm}^{-1}$ (Figure 26); for $T<T_{\mathrm{CO}}$ a mode starts to grow and becomes quite strong as the temperature is reduced further. In order to make the development more transparent, the ratios $\frac{R(T=6 \mathrm{~K})}{R(T=70 \mathrm{~K})}$ and $\frac{R(T=20 \mathrm{~K})}{R(T=70 \mathrm{~K})}$ is plotted in the right panel. The assignment of this feature is not clear at this point. Either it is a $\nu_{54}\left(b_{2 u}\right)$ which we calculate to show up at $102 \mathrm{~cm}^{-1}$ for the ionized and at $95 \mathrm{~cm}^{-1}$ for the neutral molecule [111]. Alternatively we can assigned the $110 \mathrm{~cm}^{-1}$ mode to the harmonic of $T_{b}\left(A_{g}\right)$. This implies some anharmonicity also for the lattice potential. For (TMTTF) $)_{2} \mathrm{AsF}_{6}$ we do not observe any feature at that frequency. 
Figure 26. Far-infrared reflectivity spectra of (TMTTF $)_{2} \mathrm{PF}_{6}$ at different temperatures obtained for $E \| b^{\prime}$ polarized light. A feature at $110 \mathrm{~cm}^{-1}$ gradually develops as the temperature is reduced below $T_{\mathrm{CO}}$. On the right side the reflectivity at $T=6$ and $20 \mathrm{~K}$ is normalized to the $T=70 \mathrm{~K}$ values.
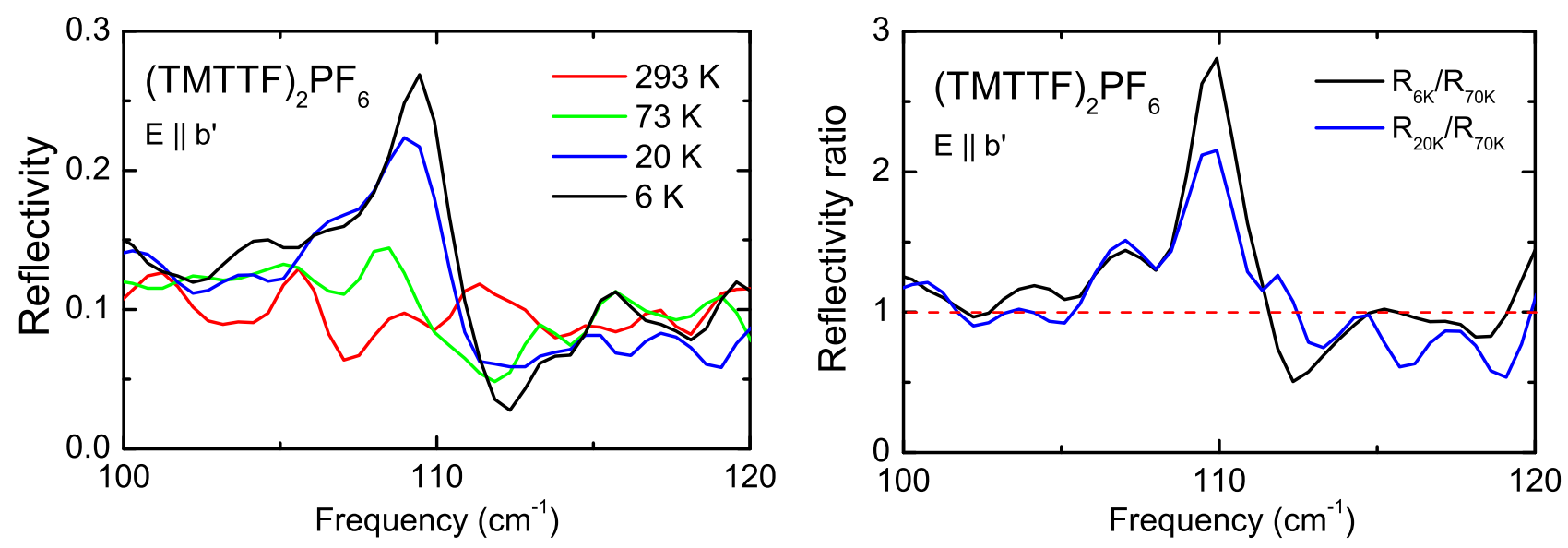

Finally, we would like to note that none of these infrared bands can be assigned to translational modes involving anion motion against the (TMTTF $)_{2}$ dimer. In fact, they always show up below $T_{\mathrm{CO}}$ when inversion symmetry is removed, while anion translations should already be infrared active even in centrosymmetric systems. The fact that these translational ionic modes are missing in the infrared spectra has been also reported for the isostructural Bechgaard (TMTSF) ${ }_{2} X$ salts by Eldridge et al. [112], whose band assignments was supported by isotope-substitution shifts. Of course, we cannot rule out the possibility that these modes occur at energies lower than $40 \mathrm{~cm}^{-1}$ due to their massive ionic components and the relatively large and soft anion cages.

\subsection{Low-Frequency Raman Spectra}

Polarized Raman spectra of $(\mathrm{TMTTF})_{2} \mathrm{AsF}_{6}$ collected with the $752 \mathrm{~nm}$ laser line at different temperatures are displayed in Figure 27. In $(a, a)$ polarization a strong $A_{g}$ band occurs around $48 \mathrm{~cm}^{-1}$ at room temperature which shows a large thermal shift up to $60 \mathrm{~cm}^{-1}$ on cooling to $T=120 \mathrm{~K}$. For the polarization $\left(b^{\prime}, a\right)$ two weaker $B_{g}$ bands are observed around 41 and $77 \mathrm{~cm}^{-1}$; the very weak band located at $77 \mathrm{~cm}^{-1}$ is further confirmed by the resonant Raman spectra reported in Figure 28, where this mode gains strong resonance enhancement from the $647 \mathrm{~nm}$ laser line and shifts up to $84 \mathrm{~cm}^{-1}$ on cooling at $T=80 \mathrm{~K}$. In resonant conditions the scattering mechanism is mainly governed by the electron-phonon interaction term; therefore we can safely assign this $B_{g}$ band to the translation mode $T_{a}$, that is a Peierls-like phonon, which is able to modulate the overlap between molecular orbitals. The strong $A_{g}$ band at $48 \mathrm{~cm}^{-1}$ is assigned to another translation mode $\left(T_{b}\right.$ or $T_{c}$ ), while the $B_{g}$ band around $41 \mathrm{~cm}^{-1}$ might be assigned to one of the librations $\left(R_{b}^{+}, R_{c}^{+}\right)$according to Table 5; however, it is more likely that the feature belongs to a low-frequency internal mode (molecular vibration) because it exhibits only a very small thermal shift. In contrast to lattice vibrations, molecular vibrations are only weakly affected by the thermal lattice contraction. 
Figure 27. Low-frequency Raman spectra of $(\mathrm{TMTTF})_{2} \mathrm{AsF}_{6}$ in the polarization $(a, a)$ and $\left(b^{\prime}, a\right)$ obtained at different temperatures using the $\lambda=752 \mathrm{~nm}$ laser line. Note the different intensity scale: $B_{g}$ spectra are two times weaker than $A_{g}$ ones.
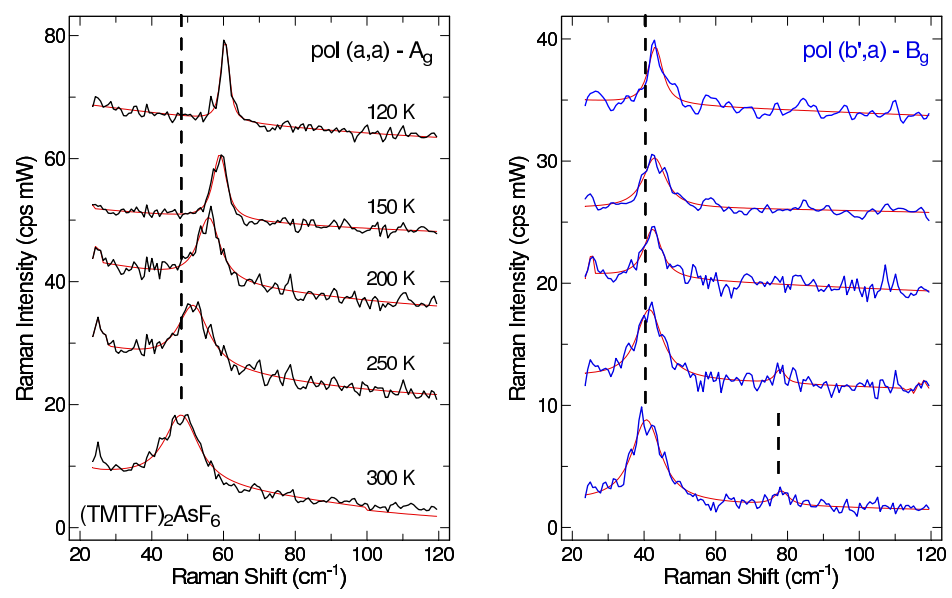

Figure 28. Unpolarized resonant Raman spectra of (TMTTF $)_{2} \mathrm{AsF}_{6}$ as a function of temperature taken with $\lambda=647 \mathrm{~nm}$.

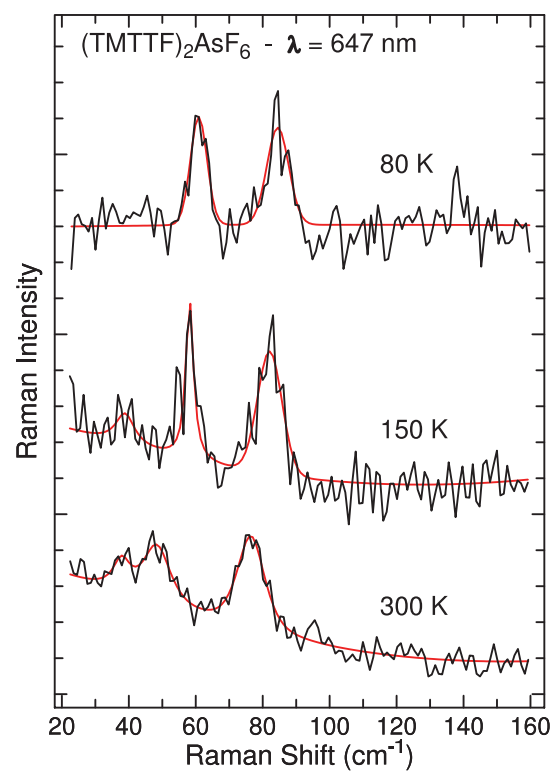

In Section 5.2 we have seen that lattice modes of (TMTTF) $)_{2} X$ become infrared active in the ferroelectric charge-ordered state. In Figure 29 we plot the representative Raman spectra of $(\mathrm{TMTTF})_{2} \mathrm{AsF}_{6}$ at low temperature $(T=15 \mathrm{~K})$. In the left panel we compare spectra obtained on two samples and with different laser power, while in the right panel we report polarized Raman spectra measured with two different laser lines [resonant $(\lambda=647 \mathrm{~nm})$ and nearly resonant $(676 \mathrm{~nm})$ ]. Five sharp bands are observed below $100 \mathrm{~cm}^{-1}$, and a cluster of broad bands around $110-130 \mathrm{~cm}^{-1}$. In Table 6 we summarize their frequencies and polarization dependences. 
Figure 29. Left panel: $\lambda=647 \mathrm{~nm}$ Raman spectra of (TMTTF $)_{2} \mathrm{AsF}_{6}$ at $15 \mathrm{~K}$. Laser power has been reduced in order to minimize sample heating. Right panel: Polarized Raman spectra at $15 \mathrm{~K}$.
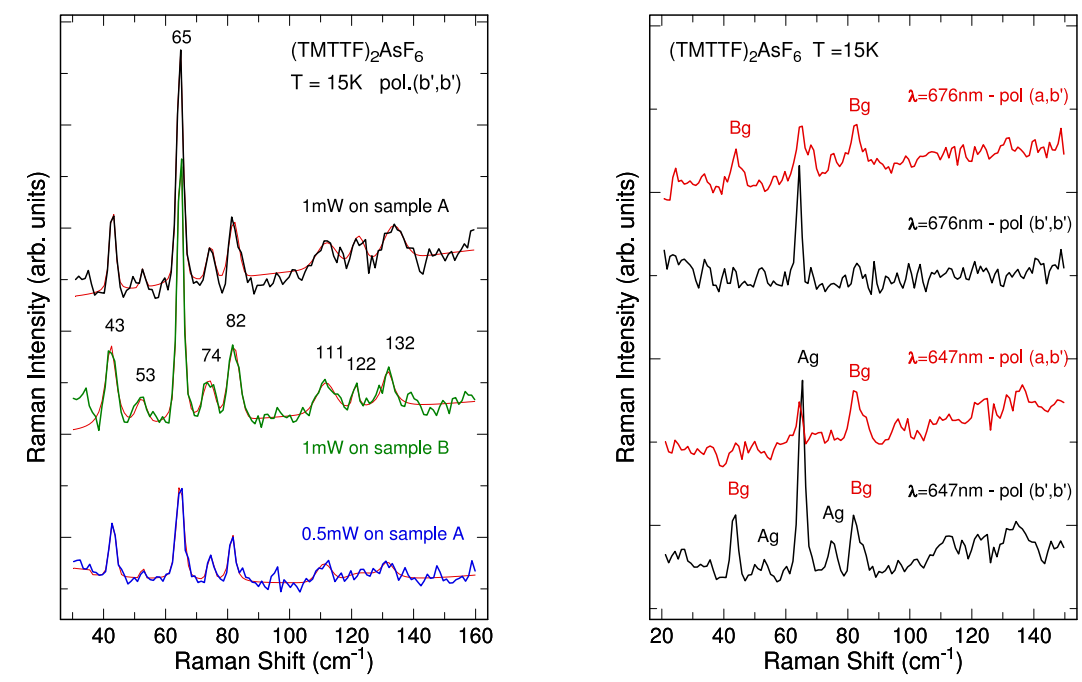

Table 6. Experimental frequencies and assignments of the (TMTTF) ${ }_{2} \mathrm{AsF}_{6}$ lattice modes obtained from our Raman and infrared measurements at room temperature and $T \approx 15 \mathrm{~K}$. Here Sym. denotes the symmetry of the modes and Pol. their polarization.

\begin{tabular}{|c|c|c|c|c|c|c|}
\hline \multirow{2}{*}{\multicolumn{2}{|c|}{$\begin{array}{c}300 \mathrm{~K} \\
\text { Raman }\end{array}$}} & \multicolumn{4}{|c|}{$15 \mathrm{~K}$} & \multirow{3}{*}{ Assignment } \\
\hline & & \multicolumn{2}{|c|}{ Raman } & \multicolumn{2}{|c|}{ infrared } & \\
\hline$\nu\left(\mathrm{cm}^{-1}\right)$ & Sym. & $\nu\left(\mathrm{cm}^{-1}\right)$ & Pol. & $\nu\left(\mathrm{cm}^{-}\right.$ & Pol. & \\
\hline 41 & $B_{g}$ & 43 & $a b^{\prime}$ & - & & $R_{b}^{+}$or int. \\
\hline- & & 53 & $b^{\prime} b^{\prime}$ & 54 & $b^{\prime}$ & $T_{b}$ \\
\hline 48 & $A_{g}$ & 65 & $a a, b^{\prime} b^{\prime}$ & 66 & $b^{\prime}$ & $T_{c}$ \\
\hline- & & 74 & $b^{\prime} b^{\prime}$ & 75 & $a$ & \\
\hline 77 & $B_{g}$ & 83 & $a b^{\prime}$ & 85 & $a$ & $T_{a}$ \\
\hline
\end{tabular}

The mode observed around $83 \mathrm{~cm}^{-1}$ in Raman spectra ( $a b^{\prime}$-polarization) has already been assigned to the $T_{a}$ translation mode, in agreement with its infrared polarization parallel to the $a$-axis (see Figure 23). The $A_{g}$ Raman bands at 53 and $65 \mathrm{~cm}^{-1}$ correspond to infrared bands observed for the perpendicular polarization ( $E \| b^{\prime}$-axis). The correspondence of the strong Raman band at $65 \mathrm{~cm}^{-1}$, assigned to a translation mode $\left(T_{b}\right.$ or $\left.T_{c}\right)$, to the weaker infrared band along the $b^{\prime}$-axis suggests a smaller projection along this direction; therefore we identify the $65 \mathrm{~cm}^{-1}$ mode as the $T_{c}$ translation mode. Correspondingly the mode at $53 \mathrm{~cm}^{-1}$ is assigned to the remaining translation mode, $T_{b}$. The third translation $\left(T_{a}\right)$ corresponds to the dimerization mode and shows up as a strong feature around $85 \mathrm{~cm}^{-1}$ in the infrared spectra for $a$ polarization. 
In order to support our phonon assignments, we have performed density functional (DFT) calculations on a (TMTTF $)_{2}^{+}$dimer, using the PBE0 hybrid functional which performs well on dimeric noncovalent interactions [113]. The vibrational analysis have been performed relaxing the dimer coordinates to the equilibrium geometry, by imposing only the constraint on intra-dimer distance (fixed at the crystal value of $3.416 \AA$ ) to simulate the crystal packing effect along the $a$-direction. The mode represented in Figure 30, with a strong Peierls-like $T_{a}$ character, is calculated at $82 \mathrm{~cm}^{-1}$ in perfect agreement with experiments, while $T_{b}$ and $T_{c}$ translation modes are calculated at 66 and $19 \mathrm{~cm}^{-1}$, respectively.

Figure 30. The Peierls-like mode of $T_{a}$ character represents the vibrations of the TMTTF molecules against each other; it is calculated at $82.3 \mathrm{~cm}^{-1}$ using DFT method.

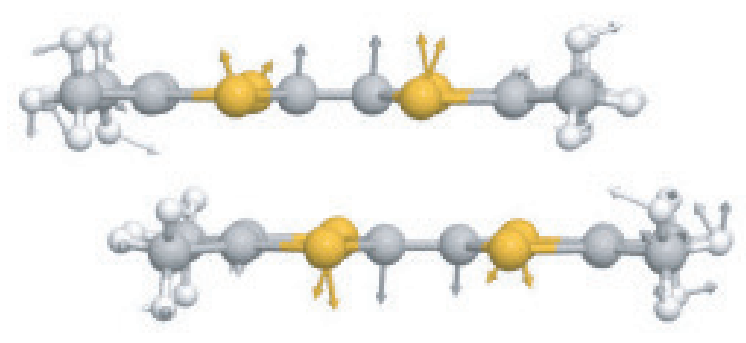

Although in our analysis we have focussed on (TMTTF $)_{2} \mathrm{AsF}_{6}$, similar observations have been made on (TMTTF) $)_{2} \mathrm{PF}_{6}$ (see Table 7). In Figure 24 the low-temperature Raman spectra are compared to each other. Some differences in the spectra should be noted, for example, a new infrared and Raman band occurs around $68 \mathrm{~cm}^{-1}$ which could not be assigned ultimately but might be a signature of the intramolecular out-of plane vibration related to the boat configuration of the TMTTF molecules [109], as discussed in the previous Subsection 5.2. This low-frequency mode is linked to the charge transfer and not observed in strongly charge-ordered systems, such as (TMTTF) ${ }_{2} \mathrm{AsF}_{6}$. The main conclusions drawn for (TMTTF) $)_{2} \mathrm{AsF}_{6}$, however, can be safely extended also to (TMTTF) ${ }_{2} \mathrm{PF}_{6}$.

Table 7. Experimental frequencies and assignments of the (TMTTF $)_{2} \mathrm{PF}_{6}$ lattice modes obtained from our Raman and infrared measurements at room temperature and $T \approx 15 \mathrm{~K}$. Here Pol. denotes the polarization used.

\begin{tabular}{|c|c|c|c|c|}
\hline \multicolumn{4}{|c|}{$15 \mathrm{~K}$} & \multirow{3}{*}{ Assignment } \\
\hline \multicolumn{2}{|c|}{ Raman } & \multicolumn{2}{|c|}{ infrared } & \\
\hline$\nu\left(\mathrm{cm}^{-1}\right)$ & Pol. & $\nu\left(\mathrm{cm}^{-1}\right)$ & Pol. & \\
\hline 42 & $a b^{\prime}$ & - & & $R_{b}^{+}$or int. \\
\hline 54 & $b^{\prime} b^{\prime}$ & 54 & $b^{\prime}$ & $T_{b}$ \\
\hline 60 & & - & & \\
\hline 66 & $a a, b^{\prime} b^{\prime}$ & - & & $T_{c}$ \\
\hline 69 & & 68 & $a$ & \\
\hline 82 & $a b^{\prime}$ & 83 & $a$ & $T_{a}$ \\
\hline
\end{tabular}




\section{Spin-Peierls Transition}

$(\mathrm{TMTTF})_{2} \mathrm{AsF}_{6}$ and $(\mathrm{TMTTF})_{2} \mathrm{PF}_{6}$, both undergo a spin-Peierls transition at $T_{\mathrm{SP}}=13$ and $19 \mathrm{~K}$, respectively. ESR experiments [46,47] clearly show the susceptibility to vanish exponentially as the triplet state forms and the lattice exhibit a tetramerization of the TMTTF chains. We can rule out a $\cdots+--++--+\cdots$ charge-distribution, since spin susceptibility does not exhibit a jump at $T_{\mathrm{SP}}$ related to such a rearrangement and symmetry change as observed below the discontinuous anion ordering transition in TMTTF salts with tetrahedral anions, such as $\mathrm{ClO}_{4}^{-}, \mathrm{BF}_{4}^{-}$or $\mathrm{ReO}_{4}^{-}$. The transition from a $4 k_{F}$ charge-ordered state to the $2 k_{F}$ ground state happens due to order in the spin sector in the case of a spin-Peierls transition, while the anion order also affects the charge sector. Since this Peierls transition involves changes in the lattice, we have carefully investigated the temperature dependence of the low-frequency modes.

As demonstrated in Figure 25 the $75 \mathrm{~cm}^{-1}$ mode of (TMTTF) $)_{2} \mathrm{AsF}_{6}$ identified already in Figure 23 splits when the temperature is lowered and $T<T_{\mathrm{SP}}$. It is not clear whether this mode is related to the feature observed in the low-temperature Raman spectra at $74 \mathrm{~cm}^{-1}$. Note, there is basically no change detected for the $T_{c}$ mode at $66 \mathrm{~cm}^{-1}$. In addition, we see a small band to evolve at $461 \mathrm{~cm}^{-1}$ as the temperature decreases below $T_{\mathrm{SP}}$.

There is theoretical and experimental evidence of a coexistence of charge order and spin-Peierls lattice distortion [10,11,29-31,42,44,79,114] with different options of the actual charge and spin patterns. In particular in both (TMTTF) $)_{2} \mathrm{AsF}_{6}$ and (TMTTF) ${ }_{2} \mathrm{PF}_{6}$ we do see a coupling of both types of ordering. The double peak found at $455 \mathrm{~cm}^{-1}$ can be assigned to the intramolecular $\nu_{35}\left(b_{1 u}\right)$ mode. This basically implies that the spin-lattice coupling at $T_{\mathrm{SP}}$ also leads to a variation of the charge distribution on the TMTTF molecules due to the tetramerization. This might also be the reason for the anomaly observed for the $\nu_{3}\left(a_{g}\right)$ mode at $T<T_{\mathrm{SP}}$. From Figure 11 it is clearly seen that the higher-frequency mode of (TMTTF $)_{2} \mathrm{AsF}_{6}$ shifts down again for the lowest temperature. Similar observations are made in our Raman spectra summarized in Figure 8. Interestingly, the behavior for (TMTTF) ${ }_{2} \mathrm{PF}_{6}$ seems to be the opposite. There also seems to be a significant variation in the coupling to the anions at $T_{\mathrm{SP}}$ as previously discussed on experimental and theoretical grounds [13,29]. Nakamura and collaborator [15,39,41] observe in their ${ }^{13} \mathrm{C}-\mathrm{NMR}$ spectra taken on (TMTTF $)_{2} \mathrm{AsF}_{6}$ that the split peaks combine into one broad peak below $T_{\mathrm{SP}}$ and interpret this as indication of a sizeable charge redistribution from charge rich to poor sites. From our vibrational spectra, we estimate this effect much smaller, not exceeding $20 \%$ of $2 \delta$ (Figure 8).

In the spectral range around $1400-1450 \mathrm{~cm}^{-1}$ we observe a reduction of the reflectivity in (TMTTF $)_{2} \mathrm{PF}_{6}$ and $(\mathrm{TMTTF})_{2} \mathrm{AsF}_{6}$ when the spin-Peierls phase is entered at $T_{\mathrm{SP}}=19$ and $13 \mathrm{~K}$, respectively, as displayed in Figure 31. In addition, a small absorption feature builds up at $1410 \mathrm{~cm}^{-1}$, which is better seen either in the reflectivity ratio $R(T=13 \mathrm{~K}): R(T=20 \mathrm{~K})$ (right frame of Figure 31) or dielectric absorption $\epsilon_{2}(\nu)$, plotted in Figure 32 for both examples.

The feature is robust and linked to the spin-Peierls phase, as proven by repeated temperature cycling. It also shows up in $(\mathrm{TMTTF})_{2} \mathrm{AsF}_{6}$ below $T_{\mathrm{SP}}=13 \mathrm{~K}$. Also the dielectric loss $\epsilon_{2}(\nu)$ is slightly reduced in the spin-Peierls phase, but around $1410 \mathrm{~cm}^{-1}$ the mode can be clearly identified. The stronger feature 
around $1398 \mathrm{~cm}^{-1}$ has been discussed in Figures 14,15 and assigned to the $\mathrm{C}-\mathrm{H}-\mathrm{C}$ bending motion of the methyl groups; it changes upon entering the charge-ordered phase.

Figure 31. Reflectivity of (TMTTF $)_{2} \mathrm{PF}_{6}$ and (TMTTF $)_{2} \mathrm{AsF}_{6}$ measured for the polarization $E \| a$ at different temperatures as indicated. In both compounds the overall value of $R(\nu)$ does not increase any more for $T<20 \mathrm{~K}$; in a narrow range between 1400 and $1450 \mathrm{~cm}^{-1}$ the reflectivity even drops and shows evidence for some mode that develops for $T<T_{\mathrm{SP}}$. The mode around $1410 \mathrm{~cm}^{-1}$ is enhanced in the reflectivity ratio $R(T=13 \mathrm{~K}) / R(T=20 \mathrm{~K})$, shown for the example of (TMTTF $)_{2} \mathrm{PF}_{6}$, in the right panel.
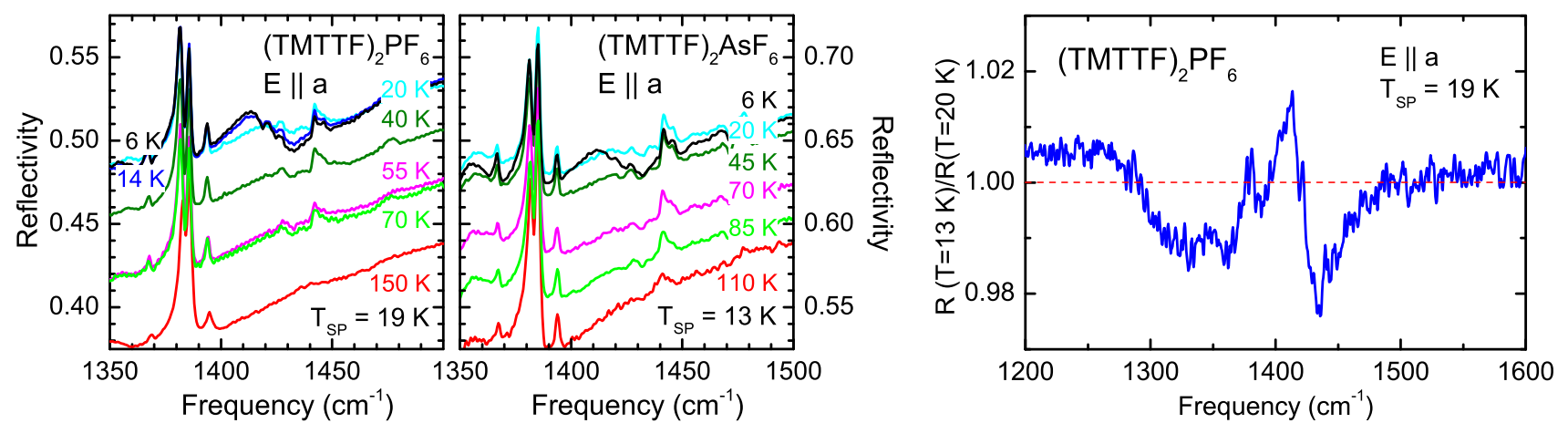

Figure 32. The dielectric losses $\epsilon_{2}(\nu)$ of (TMTTF $)_{2} \mathrm{PF}_{6}$ and (TMTTF $)_{2} \mathrm{AsF}_{6}$ exhibit a feature around $1410 \mathrm{~cm}^{-1}$ (indicated by the arrows) that builds up when the temperature drops below $T_{\mathrm{SP}}=19$ and $13 \mathrm{~K}$, respectively. The measurements have been performed with light polarized parallel to the stacks.

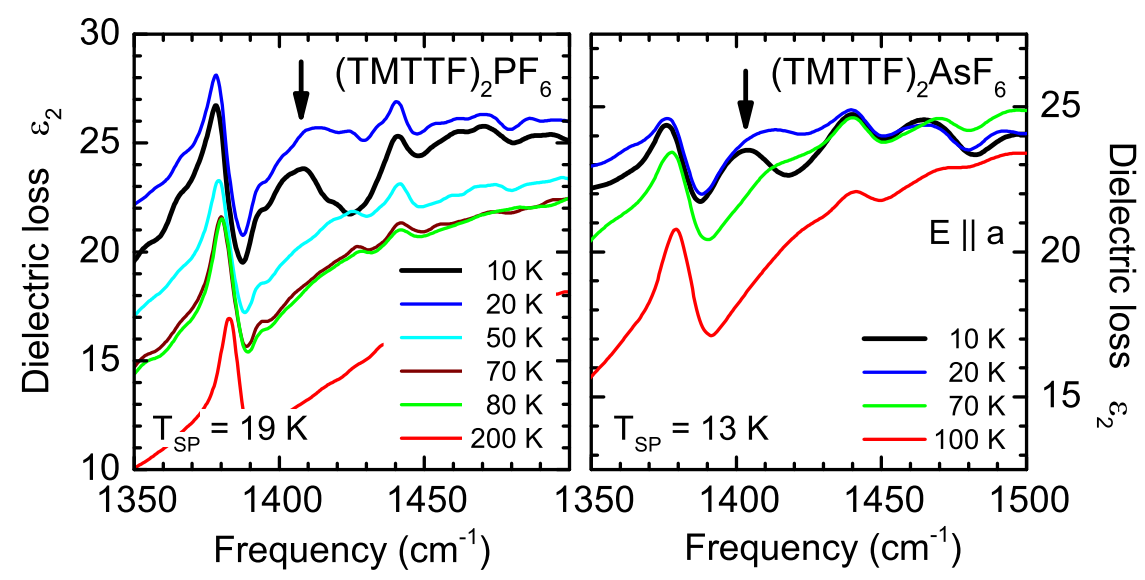

It is tempting to assign this $1410 \mathrm{~cm}^{-1}$ feature to the $\nu_{4}\left(a_{g}\right)$ mode that becomes activated due to the tetramerization. Commonly this mode is present in the infrared spectra around $1300 \mathrm{~cm}^{-1}$ (at room temperature) due to emv-coupling; i.e., independent and well above any long-range order. As seen from Table 4, the mode has the strongest emv-coupling constant. It is observed in our Raman spectra as a very strong peak at $1474 \mathrm{~cm}^{-1}$ ( $c f$. Figure 5) but does not show any splitting upon charge-ordering. Below $T_{\mathrm{SP}}$ the stacks are tetramerized, which could make the $\nu_{4}\left(a_{g}\right)$ vibrations infrared active. Low-temperature optical investigations on (TMTTF $)_{2} \mathrm{SbF}_{6}$, compared to the spin-Peierls systems, could give valuable information in this regard. For the coupling between charge order and spin-Peierls transition, see also the reviews by Pouget [91,114]. 


\section{Conclusions}

The optical investigations of the quasi-one-dimensional charge-order systems (TMTTF) $2 X$ by Raman and infrared spectroscopy yield an enormous wealth of information on the charge disproportionation amplitude and symmetry, and about the molecular interactions which may trigger the ferroelectric phase transition.

Molecular vibrations are the most suitable local probe to follow directly the charge imbalance as a function of temperature and pressure. From the totally symmetric $\nu_{3}\left(a_{g}\right)$ mode observed by Raman spectroscopy and the asymmetric $\nu_{28}\left(b_{1 u}\right)$ mode observed by infrared spectroscopy, we extract the temperature dependence of the charge disproportionation and find approximately a mean-field behavior (Figures 8,12). At low temperatures $2 \delta$ amounts to $0.15 e, 0.19 e$ and $0.29 e$ for $\left(\mathrm{TMTTF}_{2} \mathrm{PF}_{6}\right.$, $(\mathrm{TMTTF})_{2} \mathrm{AsF}_{6}$ and $(\mathrm{TMTTF})_{2} \mathrm{SbF}_{6}$, respectively. This is significantly lower than previous estimates from NMR experiments, as summarized in Table 3.

A careful analysis of the vibrational features involving the methyl groups of TMTTF and the hexafluoride anions evidences the important mutual coupling that is modified at the charge-order transition in addition to the usual change with temperature. The splitting of the $\mathrm{C}-\mathrm{C}-\mathrm{H}$ bending modes at $1000 \mathrm{~cm}^{-1}$ and at $1080 \mathrm{~cm}^{-1}$ reveals a modification of the coupling to the anions related to the broken inversion symmetry. The anions become distorted and the degeneracy of their $\nu_{4}\left(t_{1 u}\right)$ mode is lifted. This is more an effect of temperature (e.g., thermal contraction) than charge order.

Additional features are identified that are caused by the anharmonic potential. The spin-Peierls transition entails additional modifications in the charge distribution. To complete the discussion, we also add the vibrational frequencies and eigenvectors based on ab initio quantum-chemical calculations.

From the analysis of our infrared and Raman spectra we have obtained an almost complete understanding of the lattice dynamics of the (TMTTF) $)_{2} X$ systems. Above the ferroelectric transition temperature a Peierls-like mode $T_{a}$, characterized by a strong resonant behavior, have been identified in Raman spectra. Below the transition, in the charge-ordered state, this mode becomes also infrared active with polarization along the $a$-axis and marks the loss of the dimer inversion symmetry due to the site-charge redistribution. Similarly, the identification of the other translation modes, $T_{b}$ and $T_{c}$, in infrared and Raman spectra completes the picture and confirm the original hypothesis of a charge-ordered state with a $\cdots+-+-+-\cdots$ charge-distribution pattern.

\section{Acknowledgements}

Many valuable discussions with S. Brown, N. Drichko, A. Girlando, A. Pashkin, J.-P. Pouget and E. Rose are acknowledged. We thank G. Untereiner for the crystal growth and sample preparation. The project was supported by the Deutsche Forschungsgemeinschaft (DFG) and by the Carl-Zeiss-Stiftung. We would like to thank the bwGRiD [115] for providing the computational resources.

\section{Appendix}

We used the GAMESS package [116,117] for the $a b$ initio quantum chemical calculations. The ground state structure of the cation as well as the structure of the neutral molecule were optimized with the hybrid DFT-B3LYP functional and the $6-31+\mathrm{G}(d, p)$ basis set yielding good results for organic 
molecules [118]. The calculations were carried out for the isolated molecule with the $\mathrm{C}_{1}$ and the $\mathrm{D}_{2 h}$ symmetry suggested by Meneghetti et al. [59]. For the optimization we used the standard grid and a tight geometry optimization. The analysis of the cation and the neutral molecule symmetry-after the optimization with the initial symmetry $\mathrm{C}_{1}$-resides in the $\mathrm{C}_{2}$ symmetry whereas by increasing the tolerance parameters yielding also the $\mathrm{D}_{2 h}$ symmetry. Our findings support Meneghetti and coworkers who assumed that the original geometry only deviates partially from the $\mathrm{D}_{2 h}$ symmetry. All received vibrational frequencies are positive verifying that the equilibrium structure has been obtained.

The neutral molecule distorts in a boat-like confirmation (left panel Figure A1) and the cation exhibits a flat structure (right panel Figure A1) being often observed in other organic molecules [119-121]. The $\mathrm{D}_{2 h}$ symmetry for the cation results in four imaginary frequencies, respectively in one imaginary frequency for the neutral molecule indicating that the equilibrium ground state geometry for both is $\mathrm{C}_{2}$ and not $\mathrm{D}_{2 h}$.

Figure A1. On the left picture, the boat-like equilibrium geometry of the $\mathrm{TMTTF}^{0}$ molecule.
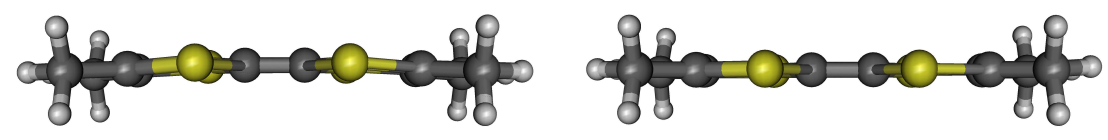

In Tables A1,A2 we report the calculated and the experimentally determined frequencies [59] of the gerade and ungerade modes, respectively, for $\mathrm{TMTTF}^{0}$ and $\mathrm{TMTTF}^{+}$with the $\mathrm{C}_{2}$ symmetry. We used two different scaling factors for the high-frequency vibrations 0.9679 (above $1300 \mathrm{~cm}^{-1}$ ) and for the lowfrequency modes 1.01 which has been actually determined for the triple- $\zeta$ basis set $6-311+\mathrm{G}(d, p)[122]$. The $a_{g}, a_{u}, b_{3 g}$ and $b_{3 u}$ modes in $\mathrm{D}_{2 h}$ reduce to A modes in $\mathrm{C}_{2}$. The $\mathrm{B}$ modes in $\mathrm{C}_{2}$ correlate with $b_{1 g}$, $b_{1 u}, b_{2 g}$ and $b_{2 u}$. For better comparison with the results of Meneghetti et al. [59] and further clarification, we sorted the normal modes according to the eight irreducible representations of the $\mathrm{D}_{2 h}$ group.

Table A1. Calculated frequencies (in $\mathrm{cm}^{-1}$ ) and infrared intensities (given in $\mathrm{D}^{2} \mathrm{amu}^{-1} \AA^{-2}$ ) of the gerade molecular modes of $\mathrm{TMTTF}^{0}$ and $\mathrm{TMTTF}^{+}$. The experimental values are extracted from [59]. The corresponding eigenvectors of the modes are displayed in Figure A2. The following abbreviations are used: Int.: Intensity, asym: asymmetric, sym: symmetric, def: deformation, ip: in-plane, oop: out-of-plane, tor: torsion, str: stretching, brea: breathing, bend: bending.

\begin{tabular}{|c|c|c|c|c|c|c|c|c|c|c|}
\hline \multirow{2}{*}{ Symmetry } & \multirow{2}{*}{ Label } & \multicolumn{4}{|c|}{ TMTTF $^{0}$} & \multicolumn{4}{|c|}{ TMTTF $^{+}$} & \multirow{2}{*}{ Mode description } \\
\hline & & $\nu_{\exp }$ & $\nu_{\text {calc }}$ & $\nu_{\text {scaled }}$ & Int. & $\nu_{\exp }$ & $\nu_{\text {calc }}$ & $\nu_{\text {scaled }}$ & Int. & \\
\hline \multirow[t]{6}{*}{$\mathrm{a}_{g}$} & $\nu_{1}$ & 2,923 & 3,142 & 3,041 & 0.4127 & & 3,167 & 3,056 & 0.1161 & $\nu-\mathrm{CH}_{3}$ asym \\
\hline & $\nu_{2}$ & & 3,034 & 2,937 & 0.0291 & & 3,054 & 2,956 & 0.0994 & $\nu-\mathrm{CH}_{3} \mathrm{sym}$ \\
\hline & $\nu_{3}$ & 1,639 & 1,690 & 1,636 & 0.0036 & 1,567 & 1,622 & 1,570 & 0 & outer, inner $\mathrm{C}=\mathrm{C}$ str \\
\hline & $\nu_{4}$ & 1,538 & 1,596 & 1,545 & 0.0036 & 1,418 & 1,492 & 1,444 & 0.0021 & outer, inner $\mathrm{C}=\mathrm{C}$ str \\
\hline & $\nu_{5}$ & 1,432 & 1,502 & 1,454 & 0.2444 & & 1,447 & 1,400 & 0 & $\alpha-\mathrm{CH}_{3}$ asym \\
\hline & $\nu_{6}$ & & 1,434 & 1,388 & 0.006 & & 1,436 & 1,391 & 0.0015 & $\alpha-\mathrm{CH}_{3}$ sym \\
\hline
\end{tabular}


Table A1. Cont.

\begin{tabular}{|c|c|c|c|c|c|c|c|c|c|c|}
\hline \multirow{2}{*}{ Symmetry } & \multirow{2}{*}{ Label } & \multicolumn{4}{|c|}{ TMTTF $^{0}$} & \multicolumn{4}{|c|}{ TMTTF $^{+}$} & \multirow{2}{*}{ Mode description } \\
\hline & & $\nu_{\exp }$ & $\nu_{\text {calc }}$ & $\nu_{\text {scaled }}$ & Int. & $\nu_{\exp }$ & $\nu_{\text {calc }}$ & $\nu_{\text {scaled }}$ & Int. & \\
\hline & $\nu_{7}$ & 1,092 & 1,103 & 1,114 & 0.051 & 1,104 & 1,101 & 1,112 & 0 & $\beta-\mathrm{CH}_{3}$ \\
\hline & $\nu_{8}$ & 934 & 940 & 949 & 0.018 & 941 & 936 & 945 & 0.0008 & $\beta-\mathrm{CH}_{3}$ \\
\hline & $\nu_{9}$ & 560 & 553 & 558 & 0.013 & 566 & 563 & 569 & 0 & $\mathrm{~S}-\mathrm{C}-\mathrm{CH}_{3} \mathrm{str}$ \\
\hline & $\nu_{10}$ & 494 & 495 & 500 & 0.0197 & 523 & 524 & 530 & 0 & $\mathrm{C}-\mathrm{S}-\mathrm{C}$ str \\
\hline & $\nu_{11}$ & 277 & 313 & 316 & 0.036 & 298 & 311 & 314 & 0.0018 & $\mathrm{CH}_{3}$ sci \\
\hline & $\nu_{12}$ & 217 & 211 & 213 & 0.0114 & 228 & 222 & 224 & 0 & rings def ip \\
\hline \multirow[t]{6}{*}{$\mathrm{b}_{1 g}$} & $\nu_{20}$ & & 3,083 & 2,984 & 0.238 & & 3,111 & 3,011 & 0.0353 & $\nu-\mathrm{CH}_{3}$ asym \\
\hline & $\nu_{21}$ & & 1,482 & 1,434 & 0.0246 & & 1,479 & 1,431 & 0.0656 & $\alpha-\mathrm{CH}_{3}$ asym \\
\hline & $\nu_{22}$ & & 1,063 & 1,073 & 0.005 & & 1,063 & 1,074 & 0.0017 & $\beta-\mathrm{CH}_{3}$ \\
\hline & $\nu_{23}$ & & 507 & 512 & 0.0006 & & 511 & 516 & 0.0002 & rings def oop \\
\hline & $\nu_{24}$ & & 176 & 178 & 0.0652 & & 151 & 153 & 0.0002 & rings def oop, $\mathrm{CH}_{3}$ tor \\
\hline & $\nu_{25}$ & & 145 & 147 & 0.005 & & 121 & 122 & 0.0002 & rings def oop, $\mathrm{CH}_{3}$ tor \\
\hline \multirow[t]{7}{*}{$\mathrm{b}_{2 g}$} & $\nu_{37}$ & & 3,082 & 2,983 & 0.2187 & & 3,113 & 3,013 & 0.0361 & $\nu-\mathrm{CH}_{3}$ asym \\
\hline & $\nu_{38}$ & & 1,488 & 1,440 & 0.4105 & & 1,487 & 1,439 & 0.0418 & $\alpha-\mathrm{CH}_{3}$ asym \\
\hline & $\nu_{39}$ & & 1,047 & 1,057 & 0.0014 & & 1,048 & 1,058 & 0.0024 & $\beta-\mathrm{CH}_{3}$ \\
\hline & $\nu_{40}$ & & 517 & 522 & 0.1132 & & 507 & 512 & 0.0001 & rings def oop \\
\hline & $\nu_{41}$ & & 261 & 263 & 0.0009 & & 296 & 299 & 0.0004 & rings def oop \\
\hline & $\nu_{42}$ & & 151 & 153 & 0.0206 & & 98 & 99 & 0 & rings def oop, $\mathrm{CH}_{3}$ tor \\
\hline & $\nu_{43}$ & & 60 & 61 & 0.0684 & & 73 & 74 & 0.0018 & rings def oop, $\mathrm{CH}_{3}$ tor \\
\hline \multirow[t]{11}{*}{$\mathrm{b}_{3 g}$} & $\nu_{55}$ & & 3,133 & 3,032 & 0.12 & & 3,158 & 3,057 & 0.0079 & $\nu-\mathrm{CH}_{3}$ asym \\
\hline & $\nu_{56}$ & & 3,031 & 2,934 & 0.0205 & & 3,052 & 2,953 & 0.0651 & $\nu-\mathrm{CH}_{3} \mathrm{sym}$ \\
\hline & $\nu_{57}$ & & 1,493 & 1,445 & 0.0343 & & 1,484 & 1,436 & 0.1928 & $\alpha-\mathrm{CH}_{3}$ asym \\
\hline & $\nu_{58}$ & & 1,417 & 1,371 & 0.0015 & & 1,419 & 1,374 & 0.0453 & $\alpha-\mathrm{CH}_{3}$ sym \\
\hline & $\nu_{59}$ & & 1,188 & 1,200 & 0.0004 & & 1,194 & 1,207 & 0 & $\beta-\mathrm{CH}_{3}$ \\
\hline & $\nu_{60}$ & & 1,118 & 1,129 & 0.0305 & & 1,126 & 1,136 & 0 & $\beta-\mathrm{CH}_{3}$ \\
\hline & $\nu_{61}$ & & 985 & 995 & 0.0032 & & 1,038 & 1,049 & 0 & $\mathrm{~S}-\mathrm{C}$ str inner, $\beta-\mathrm{CH}_{3}$ \\
\hline & $\nu_{62}$ & & 743 & 751 & 0.0072 & & 763 & 770 & 0 & $\mathrm{~S}-\mathrm{C}$ str outer \\
\hline & $\nu_{63}$ & & 473 & 478 & 0.0007 & & 474 & 479 & 0 & ring def ip \\
\hline & $\nu_{64}$ & & 385 & 389 & 0.0073 & & 387 & 391 & 0 & $\mathrm{C}-\mathrm{CH}_{3} \mathrm{str}$ \\
\hline & $\nu_{65}$ & & 246 & 259 & 0.0111 & & 236 & 239 & 0.0002 & ring def ip \\
\hline
\end{tabular}


Table A2. Calculated frequencies (in cm ${ }^{-1}$ ) and infrared intensities (given in $\mathrm{D}^{2} \mathrm{amu}^{-1} \AA^{-2}$ ) of the ungerade modes of $\mathrm{TMTTF}^{0}$ and $\mathrm{TMTTF}^{+}$. The experimental values are extracted from [59]. The eigenvectors are sketched in Figures A3-A5. The following abbreviations are used: Int.: Intensity, asym: asymmetric, sym: symmetric, def: deformation, ip: in-plane, oop: out-of-plane, tor: torsion, str: stretching, brea: breathing, bend: bending.

\begin{tabular}{|c|c|c|c|c|c|c|c|c|c|c|}
\hline \multirow{2}{*}{ Symmetry } & \multirow{2}{*}{ Label } & \multicolumn{4}{|c|}{ TMTTF $^{0}$} & \multicolumn{4}{|c|}{ TMTTF $^{+}$} & \multirow{2}{*}{ Mode description } \\
\hline & & $\nu_{\exp }$ & $\nu_{\text {calc }}$ & $\nu_{\text {scaled }}$ & Int. & $\nu_{\text {exp }}$ & $\nu_{\text {calc }}$ & $\nu_{\text {scaled }}$ & Int. & \\
\hline \multirow[t]{7}{*}{$\mathrm{a}_{u}$} & $\nu_{13}$ & & 3,082 & 2,983 & 0.4242 & & 3,111 & 3,011 & 0.0447 & $\nu-\mathrm{CH}_{3}$ asym \\
\hline & $\nu_{14}$ & & 1,483 & 1,435 & 0.1007 & & 1,480 & 1,432 & 0.0551 & $\alpha-\mathrm{CH}_{3}$ asym \\
\hline & $\nu_{15}$ & & 1,064 & 1,074 & 0.0028 & & 1,063 & 1,073 & 0.0003 & $\beta-\mathrm{CH}_{3}$ \\
\hline & $\nu_{16}$ & & 505 & 510 & 0.0016 & & 501 & 506 & 0 & ring def oop \\
\hline & $\nu_{17}$ & & 177 & 179 & 0.0298 & & 147 & 149 & 0.0006 & ring def oop \\
\hline & $\nu_{18}$ & & 145 & 146 & 0.0226 & & 117 & 118 & 0.0034 & def oop, $C_{3}$ tor \\
\hline & $\nu_{19}$ & & 66 & 67 & 0.0011 & & 43 & 43 & 0.0054 & def oop \\
\hline \multirow[t]{11}{*}{$\mathrm{b}_{1 u}$} & $\nu_{26}$ & 2,912 & 3,142 & 3,041 & 0.8701 & 2,923 & 3,166 & 3,065 & 0.1837 & $\nu-\mathrm{CH}_{3}$ asym \\
\hline & $\nu_{27}$ & 2,848 & 3,034 & 2,936 & 1.7466 & 2,848 & 3,053 & 2,956 & 0.2383 & $\nu-\mathrm{CH}_{3}$ asym \\
\hline & $\nu_{28}$ & 1,627 & 1,682 & 1,628 & 0.208 & 1,547 & 1,602 & 1,551 & 8.6833 & outer $\mathrm{C}=\mathrm{C}$ str \\
\hline & $\nu_{29}$ & 1,437 & 1,502 & 1,454 & 0.3773 & 1,438 & 1,491 & 1,443 & 0.0307 & $\alpha-\mathrm{CH}_{3}$ asym \\
\hline & $\nu_{30}$ & 1,374 & 1,433 & 1,387 & 0.001 & & 1,437 & 1,391 & 0.0857 & $\alpha-\mathrm{CH}_{3}$ sym \\
\hline & $\nu_{31}$ & 1,090 & 1,101 & 1,112 & 0.6616 & 1,095 & 1,096 & 1,108 & 0.0616 & $\beta-\mathrm{CH}_{3}, \mathrm{~S}-\mathrm{C}$ str \\
\hline & $\nu_{32}$ & 935 & 941 & 950 & 0.0842 & 935 & 930 & 939 & 1.2806 & $\nu-\mathrm{CH}_{3}$ asym, $\mathrm{S}-\mathrm{C}$ str \\
\hline & $\nu_{33}$ & 780 & 778 & 786 & 0.9168 & 828 & 821 & 829 & 0.6553 & $\mathrm{C}-\mathrm{S}$ str \\
\hline & $\nu_{34}$ & 556 & 545 & 551 & 0.0573 & 557 & 551 & 556 & 0.1366 & rings brea asym \\
\hline & $\nu_{35}$ & 439 & 435 & 440 & 0.356 & 468 & 476 & 480 & 0.5027 & rings brea asym \\
\hline & $\nu_{36}$ & 236 & 307 & 310 & 0.076 & & 306 & 309 & 0.0039 & $\mathrm{C}=\mathrm{C}-\mathrm{CH}_{3}$ bend \\
\hline \multirow[t]{11}{*}{$\mathrm{b}_{2 u}$} & $\nu_{44}$ & 2,990 & 3,132 & 3,032 & 0.25 & 2,973 & 3,159 & 3,057 & 0.028 & $\nu$ - $\mathrm{CH}_{3}$ asym \\
\hline & $\nu_{45}$ & 2,848 & 3,032 & 2,934 & 1.955 & 2,853 & 3,052 & 2,954 & 0.1876 & $\nu$ - $\mathrm{CH}_{3}$ sym \\
\hline & $\nu_{46}$ & 1,437 & 1,493 & 1,445 & 0.403 & 1,438 & 1,484 & 1,436 & 0.2372 & $\alpha-\mathrm{CH}_{3}$ asym \\
\hline & $\nu_{47}$ & 1,385 & 1,418 & 1,372 & 0.015 & 1,399 & 1,420 & 1,374 & 0.0894 & $\alpha-\mathrm{CH}_{3}$ sym \\
\hline & $\nu_{48}$ & 1,183 & 1,192 & 1,203 & 0.5125 & 1,185 & 1,197 & 1,209 & 0.0754 & $\beta-\mathrm{CH}_{3}$ \\
\hline & $\nu_{49}$ & & 1,109 & 1,120 & 0.3985 & & 1,109 & 1,120 & 0.2273 & $\beta-\mathrm{CH}_{3}$ \\
\hline & $\nu_{50}$ & & 853 & 861 & 0.0333 & 875 & 907 & 917 & 0.1686 & $\mathrm{C}-\mathrm{S}$ str, $\beta-\mathrm{CH}_{3}$ \\
\hline & $\nu_{51}$ & 747 & 745 & 753 & 0.0224 & 768 & 764 & 771 & 0.0041 & $\mathrm{C}-\mathrm{S}$ str, $\mathrm{C}=\mathrm{C}-\mathrm{CH}_{3}$ benc \\
\hline & $\nu_{52}$ & & 479 & 484 & 0.11 & & 483 & 488 & 0.0384 & $\mathrm{C}-\mathrm{CH}_{3}$ str \\
\hline & $\nu_{53}$ & 332 & 337 & 341 & 0.13 & 359 & 345 & 348 & 0.0466 & def ip, $\mathrm{C}=\mathrm{C}-\mathrm{CH}_{3}$ bend \\
\hline & $\nu_{54}$ & & 80 & 81 & 0.06 & & 83 & 84 & 0.0093 & def ip \\
\hline \multirow[t]{3}{*}{$\mathrm{b}_{3 u}$} & $\nu_{66}$ & & 3,083 & 2,984 & 0.4 & & 3,112 & 3,012 & 0.0566 & $\nu-\mathrm{CH}_{3}$ asym \\
\hline & $\nu_{67}$ & & 1,488 & 1,441 & 0.4328 & & 1,487 & 1,439 & 1.02 & $\alpha-\mathrm{CH}_{3}$ asym \\
\hline & $\nu_{68}$ & & 1,048 & 1,058 & 0.0021 & & 1,049 & 1,059 & 0.0188 & $\beta-\mathrm{CH}_{3}$ \\
\hline
\end{tabular}


Table A2. Cont.

\begin{tabular}{|c|c|c|c|c|c|c|c|c|c|}
\hline \multirow{2}{*}{ Symmetry Label } & \multicolumn{4}{|c|}{ TMTTF $^{0}$} & \multicolumn{4}{|c|}{ TMTTF $^{+}$} & \multirow{2}{*}{ Mode description } \\
\hline & $\nu_{\text {exp }}$ & $\nu_{\text {calc }}$ & $\nu_{\text {scaled }}$ & Int. & $\nu_{\exp }$ & $\nu_{\text {calc }}$ & $\nu_{\text {scaled }}$ & Int. & \\
\hline$\nu_{69}$ & & 277 & 280 & 0.1603 & & 323 & 326 & 0.1897 & def ip \\
\hline$\nu_{70}$ & & 274 & 278 & 0.02038 & & 279 & 282 & 0.0463 & def oop \\
\hline$\nu_{71}$ & & 150 & 151 & 0.115 & & 76 & 76 & 0.0142 & def oop, $\mathrm{CH}_{3}$ tor \\
\hline$\nu_{72}$ & & 34 & 35 & 0.0667 & & 46 & 47 & 0.069 & def oop \\
\hline
\end{tabular}

In order to illustrate the movement of the atoms within the TMTTF molecule for the most important vibrational modes, their eigenvectors are plotted in Figure A2 and Figure A3 through Figure A5, for the gerade and ungerade modes, respectively.

Figure A2. Eigenvectors of the twelve totally symmetric $\left(a_{g}\right)$ intramolecular modes of TMTTF. (a) $\nu_{1}\left(a_{g}\right)$ mode; (b) $\nu_{2}\left(a_{g}\right)$ mode; (c) $\nu_{3}\left(a_{g}\right)$ mode; (d) $\nu_{4}\left(a_{g}\right)$ mode; (e) $\nu_{5}\left(a_{g}\right)$ mode; (f) $\nu_{6}\left(a_{g}\right)$ mode; (g) $\nu_{7}\left(a_{g}\right)$ mode; (h) $\nu_{8}\left(a_{g}\right)$ mode; (i) $\nu_{9}\left(a_{g}\right)$ mode; (j) $\nu_{10}\left(a_{g}\right)$ mode; (k) $\nu_{11}\left(a_{g}\right)$ mode; (l) $\nu_{12}\left(a_{g}\right)$ mode.

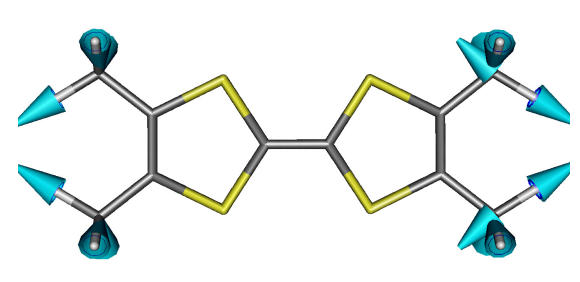

(a)

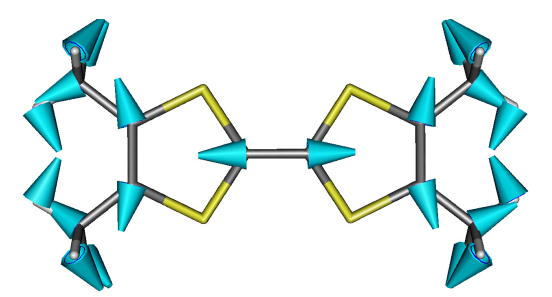

(d)

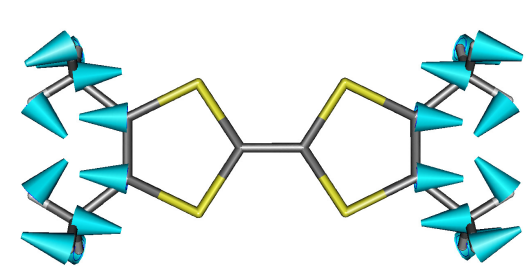

(g)

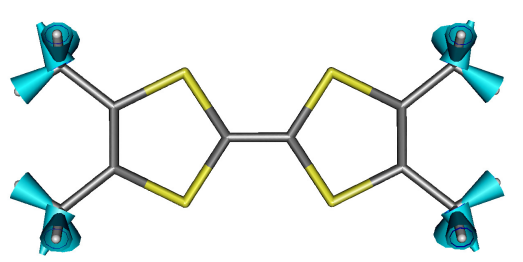

(b)

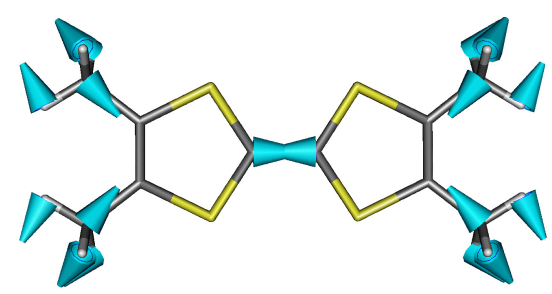

(e)

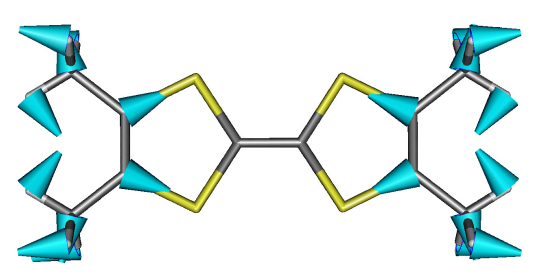

(h)

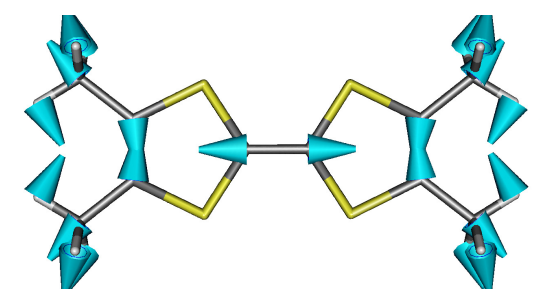

(c)

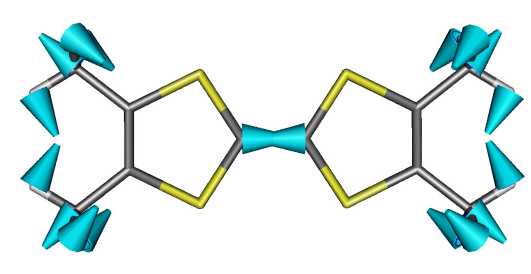

(f)

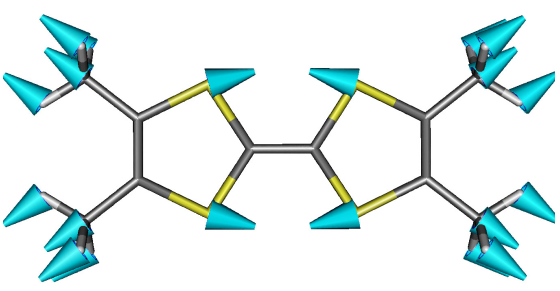

(i) 
Figure A2. Cont.

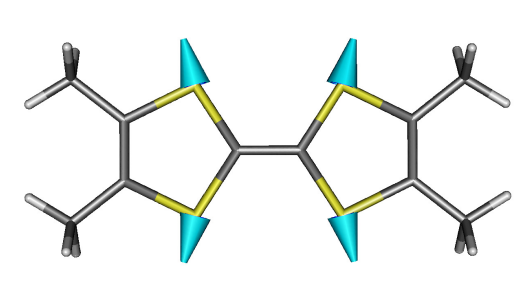

(j)

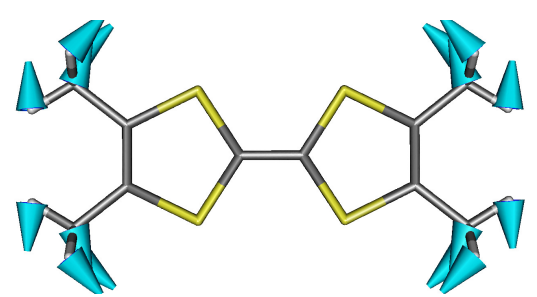

(k)

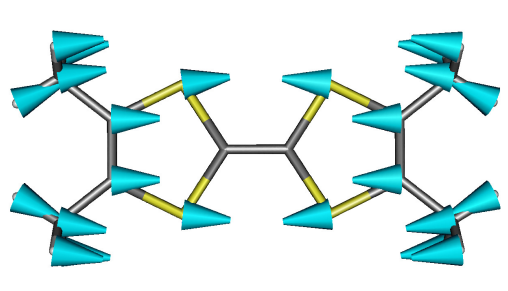

(I)

Figure A3. Eigenvectors of the eleven ungerade $\left(b_{1 u}\right)$ intramolecular modes of TMTTF. (a) $\nu_{26}\left(b_{1 u}\right)$ mode; (b) $\nu_{27}\left(b_{1 u}\right)$ mode; (c) $\nu_{28}\left(b_{1 u}\right)$ mode; (d) $\nu_{29}\left(b_{1 u}\right)$ mode; (e) $\nu_{30}\left(b_{1 u}\right)$ mode; (f) $\nu_{31}\left(b_{1 u}\right)$ mode; (g) $\nu_{32}\left(b_{1 u}\right)$ mode; (h) $\nu_{33}\left(b_{1 u}\right)$ mode; (i) $\nu_{34}\left(b_{1 u}\right)$ mode; (j) $\nu_{35}\left(b_{1 u}\right)$ mode; $(\mathbf{k}) \nu_{36}\left(b_{1 u}\right)$ mode.

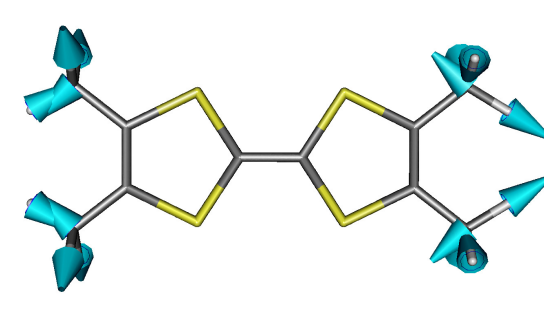

(a)

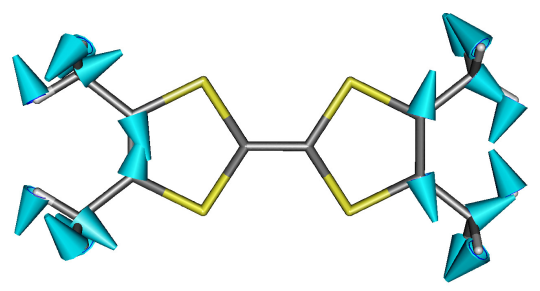

(d)

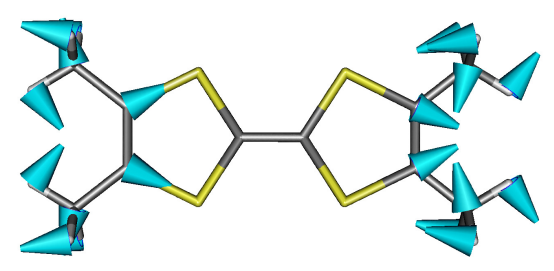

(g)

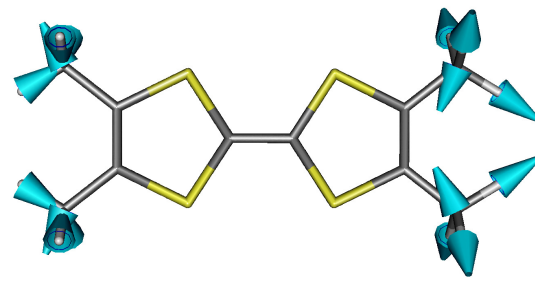

(b)

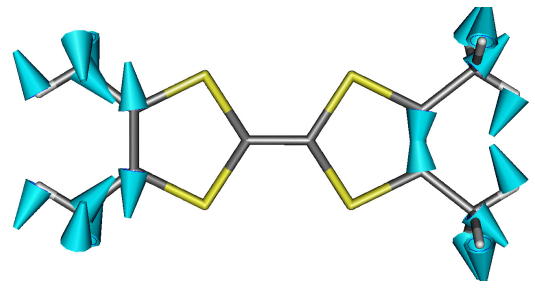

(c)

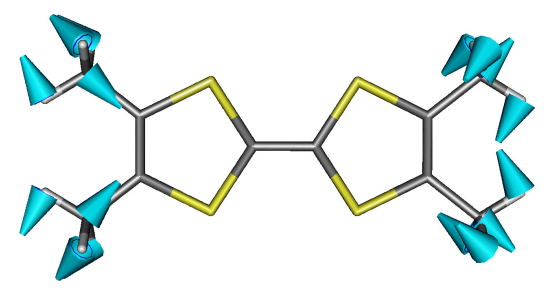

(e)

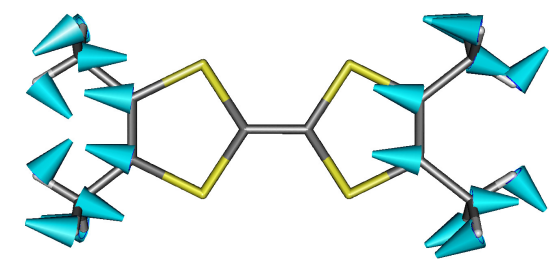

(f)

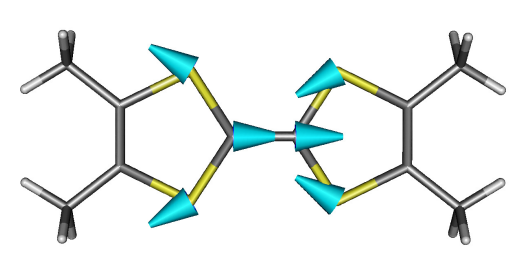

(h)

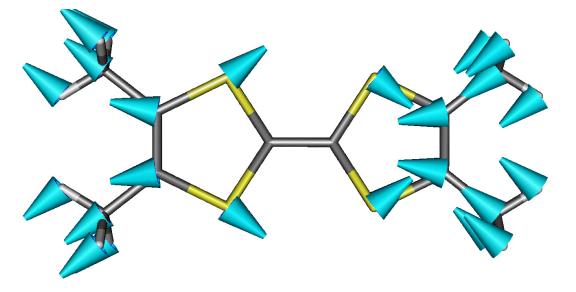

(i)

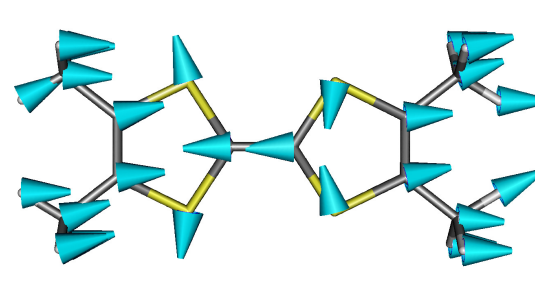

(j)

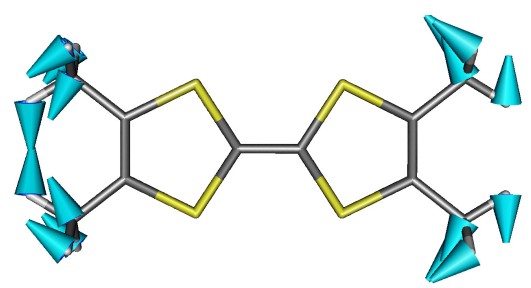

(k) 
Figure A4. Eigenvectors of the eleven ungerade $\left(b_{2 u}\right)$ intramolecular modes of TMTTF. (a) $\nu_{44}\left(b_{2 u}\right)$ mode; (b) $\nu_{45}\left(b_{2 u}\right)$ mode; (c) $\nu_{46}\left(b_{2 u}\right)$ mode; (d) $\nu_{47}\left(b_{2 u}\right)$ mode; (e) $\nu_{48}\left(b_{2 u}\right)$ mode; $(\mathbf{f}) \nu_{49}\left(b_{2 u}\right)$ mode; $(\mathbf{g}) \nu_{50}\left(b_{2 u}\right)$ mode; $(\mathbf{h}) \nu_{51}\left(b_{2 u}\right)$ mode; $(\mathbf{i}) \nu_{52}\left(b_{2 u}\right)$ mode; $(\mathbf{j}) \nu_{53}\left(b_{2 u}\right)$ mode; $(\mathbf{k}) \nu_{54}\left(b_{2 u}\right)$ mode.

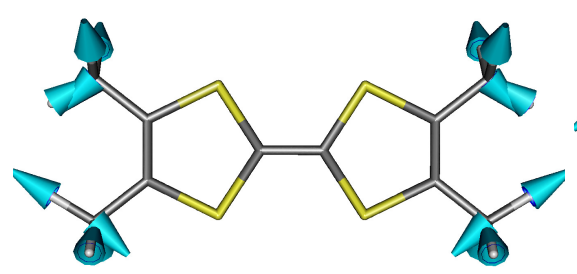

(a)

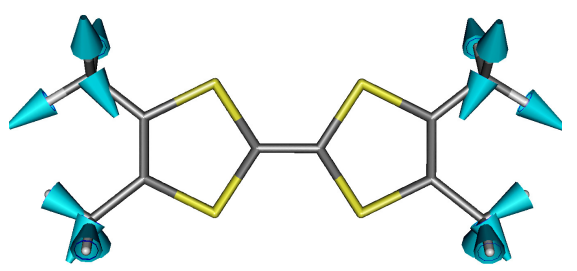

(b)

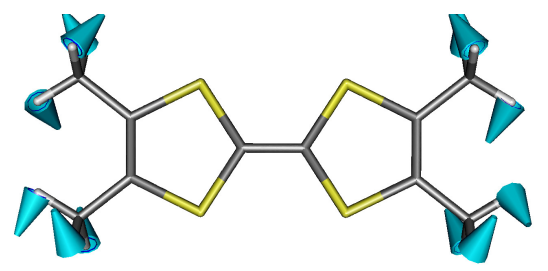

(c)

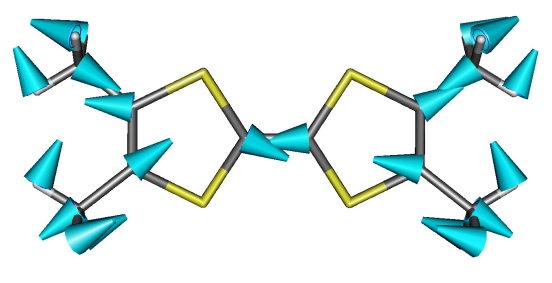

(d)

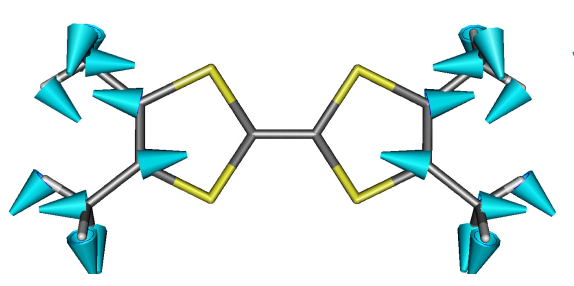

(e)

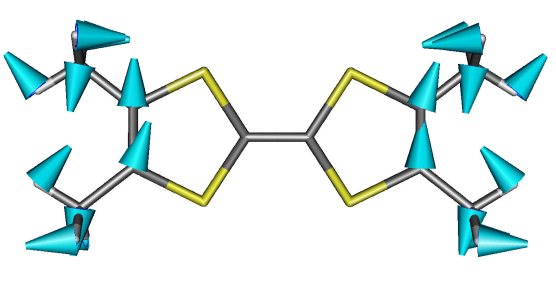

(f)

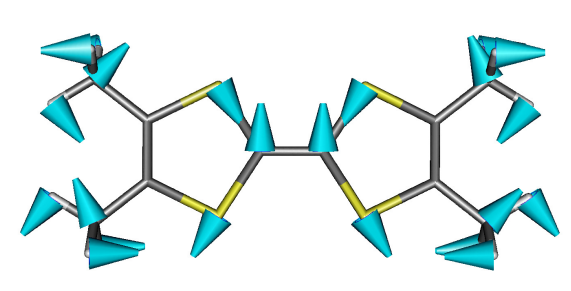

(g)

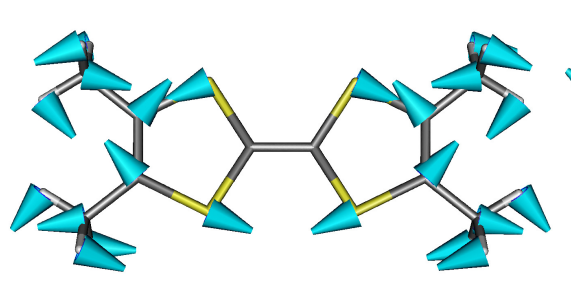

(h)

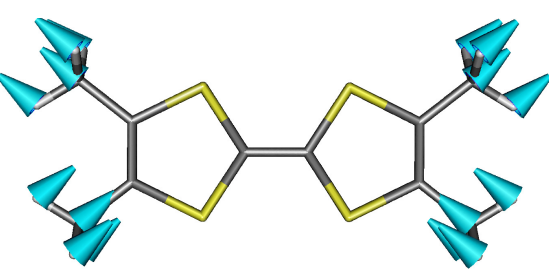

(i)

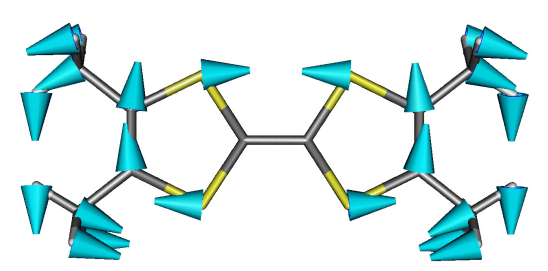

(j)

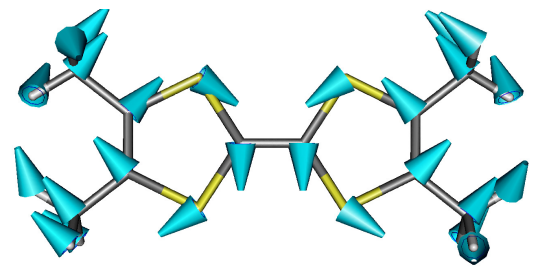

(k)

Figure A5. Eigenvectors of the seven ungerade $\left(b_{3 u}\right)$ intramolecular modes of TMTTF. (a) $\nu_{66}\left(b_{3 u}\right)$ mode; (b) $\nu_{67}\left(b_{3 u}\right)$ mode; (c) $\nu_{68}\left(b_{3 u}\right)$ mode; (d) $\nu_{69}\left(b_{3 u}\right)$ mode; (e) $\nu_{70}\left(b_{3 u}\right)$ mode; (f) $\nu_{71}\left(b_{3 u}\right)$ mode; $(\mathbf{g}) \nu_{72}\left(b_{3 u}\right)$ mode.

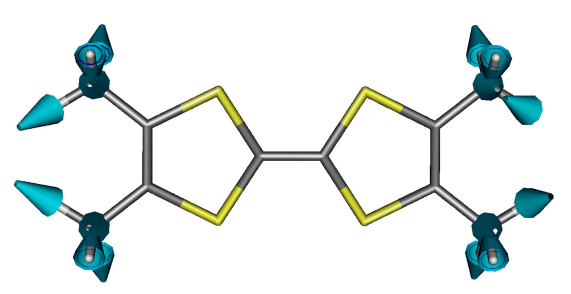

(a)

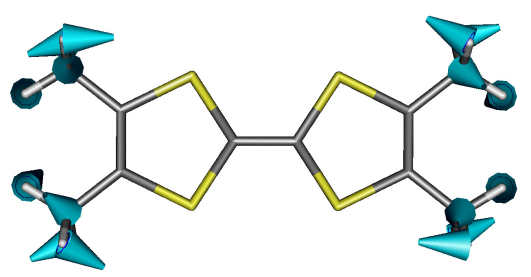

(b)

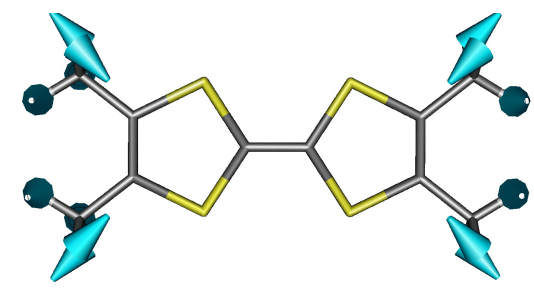

(c) 
Figure A5. Cont.

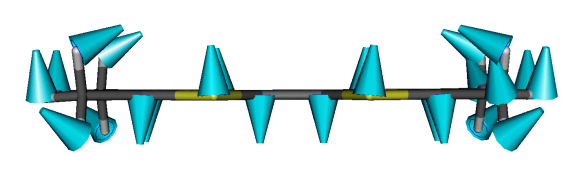

(d)

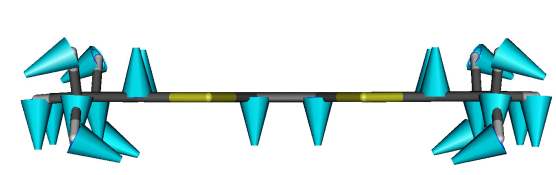

(e)

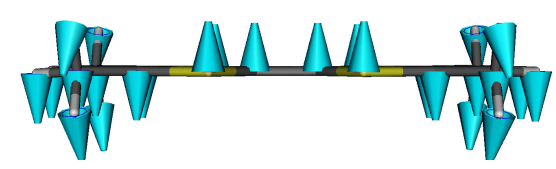

(g)

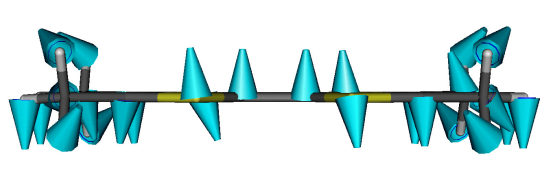

(f)

\section{References and Notes}

1. Jérome, D.; Schulz, H.J. Organic conductors and superconductors. Adv. Phys. 1982, 31, 299-490.

2. Jérome, D. The physics of organic conductors. Science 1991, 252, 1509-1514.

3. Jérome, D. Coupled Lutting Liquids. In Organic Conductors; Farges, J.-P., Ed.; Marcel Dekker: New York, NY, USA, 1994; p. 405.

4. Pouget, J.-P.; Ravy, S. Structural aspects of the Bechgaard salts and related compounds. J. Phys. (Paris) I 1996, 6, 1501-1525.

5. Dressel, M. Spin-charge separation in quasi one-dimensional organic conductors. Naturwissenschaften 2003, 90, 337-344.

6. Jérome, D. Organic conductors: From charge density wave TTF-TCNQ to superconducting (TMTSF $)_{2} \mathrm{PF}_{6}$. Chem. Rev. 2004, 104, 5565-5591.

7. Dressel, M. Ordering phenomena in quasi-one-dimensional organic conductors. Naturwissenschaften 2007, 94, 527-541.

8. Brazovskii, S. Ferroelectricity and Charge-Ordering in Quasi-1D Organic Conductors. In The Physics of Organic Superconductors and Conductors; Lebed, A., Ed.; Springer-Verlag: Berlin, Germany, 2008; pp. 313-355.

9. Coulon, C.; Parkin, S.S.P.; Laversanne, R. Structureless transition and strong localization effects in bis-tetramethyltetrahthiafulvalenium salts (TMTTF) $)_{2} X$. Phys. Rev. B 1985, 31, 3583-3587.

10. Chow, D.S.; Zamborszky, F.; Alavi, B.; Tantillo, D.J.; Baur, A.; Merlic, C.A.; Brown, S.E. Charge ordering in the TMTTF family of molecular conductors. Phys. Rev. Lett. 2000, 85, 1698-1701.

11. Zamborszky, F.; Yu, W.; Raas, W.; Brown, S.E.; Alavi, B.; Merlic, C.A.; Baur, A. Competition and coexistence of bond and charge orders in (TMTTF) ${ }_{2} \mathrm{AsF}_{6}$. Phys. Rev. B 2002, 66, 081103:1-081103:3.

12. Yu, W.; Zamborszky, F.; Alavi, B.; Baur, A.; Merlic, C.A.; Brown, S.E. Influence of charge order on the ground states of TMTTF molecular salts. J. Phys. IV France 2004, 114, 35-40. 
13. Yu, W.; Zhang, F.; Zamborszky, F.; Alavi, B.; Baur, A.; Merlic, C.A.; Brown, S.E. Electron-lattice coupling and broken symmetries of the molecular salt (TMTTF) $)_{2} \mathrm{SbF}_{6}$. Phys. Rev. B 2004, 70, 121101:1-121101:4.

14. Fujiyama, S.; Nakamura, T. Charge disproportionation in (TMTTF) ${ }_{2} \mathrm{SCN}$ observed by ${ }^{13} \mathrm{C}$ NMR. Phys. Rev. B 2004, 70, 045102:1-045102:7.

15. Fujiyama, S.; Nakamura, T. Redistribution of electronic charges in spin-Peierls state in (TMTTF $)_{2} \mathrm{AsF}_{6}$ observed by ${ }^{13} \mathrm{C}$ NMR. J. Phys. Soc. Jpn. 2006, 75, 014705:1-041705:7.

16. Nad, F.; Monceau, P.; Fabre, J.M. Low frequency dielectric permittivity of quasi-one-dimensional conductor (TMTTF) $)_{2}$ Br. Eur. Phys. J. B 1998, 3, 301-306.

17. Nad, F.; Monceau, P.; Carcel, C.; Fabre, J.M. Dielectric response of the charge-induced correlated state in the quasi-one-dimensional conductor (TMTTF) ${ }_{2} \mathrm{PF}_{6}$. Phys. Rev. B 2000; 62, 1753-1756.

18. Nad, F.; Monceau, P.; Carcel. C.; Fabre, J.M. Charge ordering phase transition in the quasi-onedimensional conductor (TMTTF) ${ }_{2} \mathrm{AsF}_{6}$. J. Phys. Condens. Matter 2000, 12, L435-L440.

19. Nad, F.; Monceau, P.; Carcel, C.; Fabre, J.M. Charge and anion ordering phase transitions in $(\mathrm{TMTTF})_{2} X$ salt conductors. J. Phys. Condens. Matter 2001, 13, L717-L722.

20. Monceau, P.; Nad, F; Brazovskii, S. Ferroelectric Mott-Hubbard phase of organic (TMTTF) ${ }_{2} X$ conductors. Phys. Rev. Lett. 2001, 86, 4080-4083.

21. Nad, F.; Monceau, P. Charge ordering and ferroelectric states in organic quasi-one-dimensional conductors. J. Phys. France 2002, 12, 133-138.

22. Nad, F; Monceau, P. Dielectric response of the charge ordered state in quasi-one-dimensional organic conductors. J. Phys. Soc. Jpn. 2006, 75, 051005:1-051005:12.

23. Laversanne, R.; Coulon, C.; Gallois, B.; Pouget, J.-P.; Moret, R. Structural and electricalproperties of $(\mathrm{TMTTF})_{2} M \mathrm{~F}_{6}$ salts $(M=\mathrm{P}, \mathrm{As}, \mathrm{Sb})$-role of the anions. J. Phys. (Paris) Lett. 1984, 45, L393-L399.

24. Granier, T.; Gallois, B.; Fritsch, A.; Ducasse, L.; Coulon, C. 135 K Crystallographic and Electronic Structure of (TNTTF $)_{2} \mathrm{SbF}_{6}$. In Lower-Dimensional Systems and Molecular Electronics; Metzger, R.M., Day, P., Papavassiliou, G.C., Eds.; Plenum Press: New York, NY, USA, 1990; p. 163.

25. Ravy, S.; Foury-Leylekian, P.; le Bolloch, D.; Pouget, J.-P.; Fabre, J.M.; Prado; R.J.; Lagarde, P. Structural instability and electronic localization in the 2:1 salts: The case of the Fabre and the (DMtTTF) ${ }_{2} \mathrm{ClO}_{4}$ salts. J. Phys. (Paris) IV 2004, 114, 81-85.

26. Seo, H.; Hotta, C.; Fukuyama, H. Toward systematic understanding of diversity of electronic properties in low-dimensional molecular solids. Chem. Rev. 2004, 104, 5005-5036.

27. Seo, H.; Merino, J.; Yoshioka, H.; Ogata, M. Theoretical aspects of charge ordering in molecular conductors. J. Phys. Soc. Jpn. 2006, 75, 051009:1-051009:21.

28. de Souza, M.; Foury-Leylekian, P.; Moradpour, A.; Pouget, J.-P.; Lang, M. Evidence for lattice effects at the charge-ordering transition in (TMTTF $)_{2} X$. Phys. Rev. Lett. 2008, 101, 216403:1-216403:4.

29. Riera, J.; Poilblanc, D. Coexistence of charge-density wave, bond-order waves, and spin-density waves in quasi-one-dimensional charge-transfer salts. Phys. Rev. B 2000, 62, 16243. 
30. Clay, R.T.; Mazumdar, S.; Campbell, D.K. Pattern of charge ordering in quasi-one-dimensional organic charge-transfer solids. Phys. Rev. B 2003, 67, 115121:1-115121:9.

31. Kuwabara, M.; Seo, H.; Ogata, M. Coexistence of charge order and spin-Peierls lattice distortion in one-dimensional organic conductors. J. Phys. Soc. Jpn. 2003, 72, 225-228.

32. Foury-Leylekian, Petit, S.; Moradpour, A.; Pouget, J.-P. Neutron scattering evidence for a lattice displacement at the charge ordering transition of (TMTTF) $)_{2} \mathrm{PF}_{6}$. Physica B 2010, 405, S95-S97.

33. Kakiuchi, T.; Wakabayashi, Y.; Sawa, H.; Itou, T.; Kanoda, K. Wigner crystallization in (DI-DCNQI $)_{2} \mathrm{Ag}$ detected by synchrotron radiation X-ray diffraction. Phys. Rev. Lett. 2007, 98, 066402:1-066402:4.

34. Zorina, L.; Simonov, S.; Meziere, C.; Canadell, E.; Suh, S.; Brown, S.E.; Foury-Leylekian, P.; Fertey, P.; Pouget, J.-P.; Batail, P. Charge ordering, symmetry and electronic structure issues and Wigner crystal structure of the quarter-filled band Mott insulators and high pressure metals $\delta$-(EDT-TTF-CONMe $)_{2} X, X=\mathrm{Br}$ and $\mathrm{AsF}_{6}$. J. Mater. Chem. 2009, 19, 6980-6994.

35. Yasin, S.; Salameh, B.; Rose, E.; Dumm, M.; Krug von Nidda, H.-A.; Loidl, A.; Ozerov, M.; Untereiner, G.; Montgomery, L.; Dressel, M. Broken magnetic symmetry due to charge-order ferroelectricity discovered in (TMTTF $)_{2} X$ salts by multifrequency ESR. Phys. Rev. B 2012, 85, 144428:1-144428:14.

36. Rose, E.; Lissner, F.; Schleid, T.; Dressel, M. Temperature dependent crystalgraphic structure of $(\mathrm{TMTTF})_{2} X$. Universität Stuttgart, Stuttgart, Germany, Unpublished work, 2012.

37. Köhler, B.; Rose, E.; Dumm, M.; Untereiner, G.; Dressel, M. Comprehensive transport studies of anisotropy and ordering phenomena in quasi-one-dimensional (TMTTF) ${ }_{2} X$ salts $\left(X=\mathrm{PF}_{6}, \mathrm{AsF}_{6}\right.$, $\mathrm{SbF}_{6} ; \mathrm{BF}_{4}, \mathrm{ClO}_{4}, \mathrm{ReO}_{4}$. Phys. Rev. B 2011, 84, 035124:1-035124:14.

38. Dressel, M.; Hesse, P.; Kirchner, S.; Untereiner, G.; Dumm, M.; Hemberger, J.; Loidl, A.; Montgomery, L. Charge and spin dynamics of TMTSF and TMTTF salts. Synth. Met. 2001, 120, 719-720.

39. Nakamura, T.; Furukawa, K.; Hara, T. Redistribution of charge in the proximity of the spin-Peierls transition: ${ }^{13} \mathrm{C}$ NMR investigation of (TMTTF) $)_{2} \mathrm{PF}_{6}$. J. Phys. Soc. Jpn. 2007, 76, 064715:1-064715:7.

40. Nakamura, T. Possible charge ordering patterns of the paramagnetic insulating states in (TMTTF $)_{2}$ X. J. Phys. Soc. Jpn. 2003, 72, 213-216.

41. Hirose, S.; Kawamoto, A.; Matsunaga, N.; Nomura, K.; Yamamoto, K.; Yakushi, K. Reexamination of ${ }^{13} \mathrm{C}-\mathrm{NMR}$ in $\left.(\mathrm{TMTTF})_{2} \mathrm{AsF}_{6}\right)$ : Comparison with infrared spectroscopy. Phys. Rev. B 2010, 81, 205107:1-205107:6.

42. Dumm, M.; Salameh, B.; Abaker, M.; Montgomery, L.K.; Dressel, M. Magnetic and optical studies of spin and charge ordering in (TMTTF $)_{2} \mathrm{AsF}_{6}$. J. Phys. (Paris) IV 2004, 114, 57-60.

43. Dumm, M.; Abaker, M.; Dressel, M. Mid-infrared response of charge-ordered quasi-1D organic conductors (TMTTF) $)_{2}$ X. J. Phys. (Paris) IV 2005, 131, 55-58.

44. Dumm, M.; Abaker, M.; Dressel, M.; Montgomery, L.K. Charge order in $(\mathrm{TMTTF})_{2} \mathrm{PF}_{6}$ investigated by infrared spectroscopy. J. Low Temp. Phys. 2006, 142, 609-612.

45. Knoblauch, T.; Dressel, M. Charge disproportionation in (TMTTF $)_{2} X\left(X=\mathrm{PF}_{6}, \mathrm{AsF}_{6}\right.$ and $\left.\mathrm{SbF}_{6}\right)$ investigated by infrared spectroscopy. Phys. Status Solidi (C) 2012, 9, 1158-1160. 
46. Salameh, B.; Yasin, S.; Dumm, M.; Untereiner, G.; Montgomery, L.; Dressel, M. Spin dynamics of the organic linear chain compounds (TMTTF) $X\left(X=\mathrm{SbF}_{6}, \mathrm{AsF}_{6}, \mathrm{BF}_{4}, \mathrm{ReO}_{4}\right.$ and $\left.\mathrm{SCN}\right)$. Phys. Rev. B 2011, 83, 205126:1-205126:8.

47. Dumm, M.; Loidl, A.; Fravel, B.W.; Starkey, K.P.; Montgomery, M.; Dressel, M. Electron spin resonance studies on the organic linear-chain compounds (TMTCF $)_{2} X\left(C=\mathrm{S}, \mathrm{Se} ; X=\mathrm{PF}_{6}, \mathrm{AsF}_{6}\right.$, $\mathrm{ClO}_{4}, \mathrm{Br}$ ). Phys. Rev. B 2000, 61, 511-521.

48. Dumm, M.; Loidl, A.; Alavi, B.; Starkey, K.P.; Montgomery, M.; Dressel, M. Comprehensive ESR study of the antiferromagnetic ground states in the one-dimensional spin systems (TMTSF) ${ }_{2} \mathrm{PF}_{6}$, (TMTSF) ${ }_{2} \mathrm{AsF}_{6}$, and (TMTTF) ${ }_{2}$ Br. Phys. Rev. B 2000, 62, 6512-6520.

49. Nagasawa, M.; Nad, F.; Monceau, P.; Fabre, J.-M. Modification of the charge ordering transition in the quasi-one-dimensional conductor (TMTTF)(2) $\mathrm{SbF}_{6}$ under pressure. Solid State Commun. 2005, 136, 262-267.

50. Nagasawa, M.; Nagasawa, T.; Ichimura, K.; Nomura, K. Anisotropic pressure effects on the charge order transition of (TMTTF) $)_{2} X$. Physica B 2010, 405, S113-S115.

51. Itoi, M.; Ishii, Y.; Takekoshi, S.; Kitano, H.; Matsubayashi, K.; Uwatoko, Y.; Nakamura, T. Completely hydrostatic pressure effect of anisotropic resistivity in the 1-D organic conductor (TMTTF $)_{2} \mathrm{SbF}_{6}$. Physica C 2010, 470, S594-S595.

52. Iwase, F.; Sugiura, K.; Furukawa, K.; Nakamura, T. ${ }^{13} \mathrm{C}$ NMR study of the magnetic properties of the quasi-one-dimensional conductor (TMTTF) $)_{2} \mathrm{SbF}_{6}$. Phys. Rev. B 2011, 84, 115140:1115140:8.

53. Schrem, K.; Rose, E.; Dressel, M. Pressure-dependent transport studies of the ordering transitions in $\left(\mathrm{TMTTF}_{2} X\right.$. Universität Stuttgart, Stuttgart, Germany, Unpublished work, 2012.

54. Pashkin, A.; Dressel, M.; Kuntscher, C.A. Pressure-induced deconfinement of the charge transport in the quasi-one-dimensional mott insulator (TMTTF) $)_{2} \mathrm{AsF}_{6}$. Phys. Rev. B 2006, 74, 165118:1-165118:10.

55. Pashkin, A.; Dressel, M.; Hanfland, M.; Kuntscher, C.A. Deconfinement transition and dimensional crossover in the Bechgaard-Fabre salts: Pressure- and temperature-dependent optical investigations. Phys. Rev. B 2010, 81, 125109:1-125109:12.

56. Galigné, J.L.; Liautard, B.; Peytavin, S.; Brun, G.; Maurin, M.; Fabre, J.-M.; Torreilles, E.; Giral, L. Crystal-structure of tetramethyltetrathiofulvalene fluoroborate at $100 \mathrm{~K}$ and roomtemperature. Acta Cryst. B 1979, 35, 1129-1135.

57. Liautard, B.; Peytavin, S.; Brun, G.; Maurin, M. Structural correlations in the series (TMTTF) ${ }_{2} X$. J. Phys. (Paris) 1982, 43, 1453-1459.

58. Kobayashi, H.; Kobayashi, A.; Sasaki, Y.; Saito, G.; Inokuchi, H. The crystal-structure of (TMTTF $)_{2} \mathrm{ReO}_{4}$. Bull. Chem. Soc. Jpn. 1984, 57, 2025-2026.

59. Meneghetti, M.; Bozio, R.; Zanon, I.; Pecile, C.; Ricotta, C.; Zanetti, M. Vibrational behavior of molecular constituents of organic superconductors-TMTTF, its radical cation and the sulfur analogs TMTTF and TMTTF ${ }^{+}$. J. Chem. Phys. 1984, 80, 6210-6224.

60. Girlando, A.; Masino, M.; Brillante, A.; Della Valle, R.G.; Venuti, E. BEDT-TTF organic superconductors: The role of phonons. Phys. Rev. B 2002, 66, 100507:1-100507:4. 
61. Bozio, R.; Pecile, C. Charge Transfer Crystals and Molecular Conductors. In Advances in Spectroscopy, 19: Spectroscopy Of Advanced Materials; Clark, R.J.H., Hester, R.E., Eds.; Wiley \& Sons: Chichester, UK, 1991; p. 1.

62. Dressel, M.; Drichko, N. Optical properties of two-dimensional organic conductors: Signatures of charge ordering and correlation effects. Chem. Rev. 2004, 104, 5689-5715.

63. Rice, M.J. Organic linear conductors as systems for study of electron-phonon interactions in organic solid-state. Phys. Rev. Lett. 1976, 37, 36-39.

64. Rice, M.J.; Pietronero, L.; Brüesch, P. Phase phonons and intramolecular electron-phonon coupling in organic linear-chain semiconductor TEA(TCNQ) $)_{2}$. Solid State Commun. 1977, 21 , 757-760.

65. Rice, M.J.; Lipari, N.O.; Strässler, S. Dimerized organic linear-chain conductors and unambiguous experimental-determination of electron-molecular-vibration coupling-constants. Phys. Rev. Lett. 1977, 39, 1359-1362.

66. Rice, M.J. Towards the experimental-determination of the fundamental microscopic parameters of organic ion-radical compounds. Solid State Commun. 1979, 31, 93-98.

67. Rice, M.J.; Yartsev, V.M.; Jacobsen, C.S. Investigation of the nature of the unpaired electron-states in the organic semiconductor $n$-methyl- $n$-ethylmorpholinium-tetracyanoquinodimethane. Phys. Rev. B 1980, 21, 3437-3446.

68. Lipari, N.O.; Duke, C.B.; Bozio, R.; Girlando, A.; Pecile, C.; Padva, A. Electron-molecularvibration coupling in 7,7,8,8-tetracyano-para-quinodimethane (TCNQ). Chem. Phys. Lett. 1976, 44, 236-240.

69. Jacobsen, C.S.; Tanner, D.B.; Bechgaard, K. Dimensionality crossover in the organic superconductor tetramethyltetraselenafulvalene hexafluorophosphate [(TMTSF $)_{2} \mathrm{PF}_{6}$ ] compounds. Phys. Rev. Lett. 1981, 46, 1142-1145.

70. Jacobsen, C.S.; Tanner, D.B.; Bechgaard, K. Optical and infrared properties of tetramethyltetraselenafulvalene $\left[(\mathrm{TMTSF})_{2} X\right]$ and tetramethyltetrathiafulvalene [(TMTTF $\left.)_{2} X\right]$ compounds. Phys. Rev. B 1983, 28, 7019-7032.

71. Degiorgi, L.; Dressel, M.; Schwartz, A.; Alavi, B. Grüner, G. Direct observation of the spin-density-wave gap in (TMTSF) ${ }_{2} \mathrm{PF}_{6}$. Phys. Rev. Lett. 1996, 76, 3838-3841.

72. Vescoli, V.; Degiorgi, L.; Henderson, W.; Grüner, G.; Starkey, K.P.; Montgomery, L.K. Dimensionality-driven insulator-to-metal transition in the Bechgaard salts. Science 1998, 281, 1181-1184.

73. Dressel, M.; Grüner, G. Electrodynamics of Solids; Cambridge University Press: Cambridge, UK, 2002.

74. Meneghetti, M.; Toffoletti, A.; Pasimeni, L. Experimental evaluation of the electronintramolecular-vibration coupling constants of tetramethyltetrathiafulvalene. Phys. Rev. B 1996, 54, 16353-16356.

75. Pedron, D.; Bozio, R.; Meneghetti, M.; Pecile, C. Electron-phonon coupling in low dimensional organic superconductors. Mol. Cryst. Liquid Cryst. 1993, 234, 161-170. 
76. Painelli, A.; Girlando, A. Electron molecular vibration (e-mv) coupling in charge-transfer compounds and its consequences on the optical-spectra-A theoretical framework. J. Chem. Phys. 1986, 84, 5655-5671.

77. Yamamoto, K.; Yakushi, K. Electron-molecular vibration coupling effect on the Raman spectrum of organic charge transfer salts. J. Phys. (Paris) IV 2004, 114, 153-155.

78. Takahashi, T.; Nogami, Y.; Yakushi, K. Charge ordering in organic conductors. J. Phys. Soc. Jpn. 2006, 75, 051008:1-051008:17.

79. Riera, J.; Poilblanc, D. Influence of the anion potential on the charge ordering in quasi-one-dimensional charge-transfer salts. Phys. Rev. B 2001, 63, 241102:1-241102:4.

80. Yamamoto, T.; Uruichi, M.; Yamamoto, K.; Yakushi, K.; Kawamoto, A.; Taniguchi, H. Examination of the charge-sensitive vibrational modes in bis(ethylenedithio)tetrathiafulvalene. J. Phys. Chem. B 2005, 109, 15226-15235.

81. Drichko, N.; Kaiser, S.; Sun, Y.; Clauss, C.; Dressel, M.; Mori, H.; Schlueter, J.A.; Zhilyaeva, E.; Torunova, S.A.; Lyubovskaya, R. Evidence for charge order in organic superconductors obtained by vibrational spectroscopy. Physica B 2009, 404, 490-493.

82. Girlando, A. Charge sensitive vibrations and electron-molecular vibration coupling in Bis(ethylenedithio)-tetrathiafulvalene (BEDT-TTF). J. Phys. Chem. C 2011, 115, 19371-19378.

83. Girlando, A.; Masino, M.; Kaiser, S.; Sun, Y.; Drichko, N.; Dressel, M.; Mori, H. Spectroscopic characterization of charge order fluctuations in BEDT-TTF metals and superconductors. Phys. Status Solidi (B) 2012, 249, 953-956.

84. de Souza, M.; Hofmann, D.; Foury-Leylekian, P.; Moradpour, A.; Pouget, J.-P.; Lang, M. Exploring the charge-order transition in (TMTTF) $)_{2} X$ via thermal expansion measurements. Physica B 2010, 405, S92-S94.

85. Eijt, S.; Schut, H. Proceedings of the International Workshop on Positron Studies of Defects 2011 (PSD-11). In Physica B; Elsevier: Amsterdam, The Netherland, 2012; volume 407.

86. Hildebrandt, S.; Stass, I. Physica Status Solidi (B); Wiley \& Sons, Inc.: Hoboken, NJ, USA, 2012; volume 249, pp. 637-863.

87. Special Issue: 9th International Symposium on Crystalline Organic Metals, Superconductors and Ferromagnets (ISCOM 2011). In Physica Status Solidi (C); Wiley \& Sons, Inc.: Hoboken, NJ, USA, 2012; volume 5, pp. 1115-1344.

88. Kistenmacher, T.J. Cavity sice versus anion size in (TMTSF) ${ }_{2} X$ salts - possible implications for the uniqueness of (TMTSF) $)_{2} \mathrm{ClO}_{4}$. Solid State Commun. 1984, 50, 729-733.

89. Kistenmacher, T. Anion-donor coupling in (TMTSF) $)_{2} X$ salts-Symmetry considerations. Solid State Commun. 1984, 51, 931-934.

90. Beno, M.A.; Blackman, G.S.; Leung, P.C.W.; Williams, J.M. Hydrogen bond formation and anion ordering in superconducting (TMTSF) $)_{2} \mathrm{ClO}_{4}$ and (TMTSF) $)_{2} \mathrm{AsF}_{6}$. Solid State Commun. 1983, 48, 99-103.

91. Pouget, J.-P. Structural aspects of the Bechgaard and Fabre salts: An update Crystals 2012, doi:10.3390/cryst2020466.

92. Scott, J.C.; Pedersen, H.J.; Bechgaard, K. Proton NMR in the organic conductor tetramethyltetraselenafulvalinium hexafluorophosphate. Phys. Rev. B 1981, 24, 475-477. 
93. McBrierty, V.J.; Douglass, D.C.; Wudl, F.; Aharon-Shalom, E. Nuclear resonance and relaxation in ditetramethyltetraselenafulvalenium salts. Phys. Rev. B 1982, 26, 4805-4809.

94. Kim, S.H.; Lee, K.W.; Lee, C.E.; Kang, W.; Hong, K.S. Decoupled critical dynamics of the TMTSF donor molecules in (TMTSF) $)_{2} X$ organic superconductors. Phys. Rev. B 2006, 73, 012505:1-012505:3.

95. Jankowski, D.; Świetlik, R.; Reinheimer, E.W.; Fourmigué, M. Involvement of weak C-H X hydrogen bonds in metal-to-semiconductor regime change in one-dimensional organic conductors (o-DMTTF $)_{2} X(X=\mathrm{Cl}, \mathrm{Br}$, and I): Combined IR and Raman studies. J. Raman Spectrosc. 2011, $42,1518-1527$.

96. Thorup, N.; Rindorf, G.; Soling, H.; Bechgaard, K. The structure of Di(2,3,6,7-tetramethyl1,4,5,8-tetraselenafulvalenium) Hexafluorophosphate, (TMTSF) ${ }_{2} \mathrm{PF}_{6}$, the first superconducting organic solid. Acta Cryst. B 1981, 37, 1236-1240.

97. Nakamoto, K. Infrared and Raman Spectra of Inorganic and Coordination Compounds. Part A: Theory and Application in Inorganic Chemistry, 6th ed.; John Wiley \& Sons: Hoboken, NJ, USA, 2009.

98. Rose, E.; Dressel, M. Coupling between molecular chains and anions in (TMTTF $)_{2} X$ salts. Physica B 2012 407, 1787-1792.

99. Andrieux, A.; Jerome, D.; Bechgaard, K. Spin-density wave ground-state in the one-dimensional conductor (TMTSF) ${ }_{2} \mathrm{PF}_{6}$ : microscopic evidence from ${ }^{77} \mathrm{Se}$ and ${ }^{1} \mathrm{H}-\mathrm{NMR}$ experiments. J. Phys. (Paris) Lett. 1981, 42, L87-L90.

100. Furukawa, K.; Hara, T.; Nakamura, T. Anomalous temperature dependence of g-tensor in organic conductor, (TMTTF $)_{2} X\left(X=\mathrm{Br}, \mathrm{PF}_{6}\right.$, and $\left.\mathrm{SbF}_{6}\right)$. J. Phys. Soc. Jpn. 2009, 78, 104713:1-104713:6.

101. Pashkin, A.; Beyer, R.; Kuntscher, C.A.; Dressel, M. Pressure-dependent vibrational studies of $(\mathrm{TMTTF})_{2} X$. Universität Stuttgart, Stuttgart, Germany, Unpublished work, 2012.

102. Rose, E.; Loose, C.; Kortus, J.; Pashkin, A.; Kuntscher, C.A.; Ebbinghaus, S.G.; Hanfland, M.; Lissner, F; Dressel, M. Pressure Dependence of Crystal Structure and Electronic Band Structure of one-dimensional (TMTTF) $)_{2} \mathrm{PF}_{6}$. J. Phys.: Cond. Matter 2012, in press.

103. Yamamoto, K.; Kowalska, A.A.; Yue, Y.; Yakushi, K. Vibronic activation of molecular overtones in the infrared spectra of charge-ordered organic conductors. Phys. Rev. B 2011, 84, 064306:1-064306:13.

104. Ivek, T.; Kotrin-Hamzić;Milat, O.; Tomić, S.; Clauss, C; Drichko, N.; Schweitzer, D.; Dressel, M. Electrodynamic response of the charge ordering phase: Dielectric and optical studies of $\alpha$ (BEDT-TTF $)_{2} \mathrm{I}_{3}$. Phys. Rev. B 2011, 83, 165128:1-165128:13.

105. Yamamoto, K.; Kowalska, A.A.; Yue, Y.; Yakushi, K. E-mv coupling of vibrational overtone in organic conductors: Relationship to optical nonlinearities and ferroelectricity. Physica B 2012, 407, 1775-1778.

106. Antal, Á.; Knoblauch, T.; Dressel, M.; Batail, P.; Drichko, N. Signatures of optical excitaitons of domain walls in infrared properties of 1D quarter-filled Wigner lattice compounds $\delta$-[(EDT-TTF)$\left.\mathrm{CONMe}_{2}\right]_{2} X, X=\mathrm{AsF}_{6}$, Br. Phys. Rev. B 2012, in press. 
107. Iwase, F.; Sugiura, K.; Furukawa, K.; Nakamura, T. Electronic properties of a TMTTF-family salt, (TMTTF) $)_{2} \mathrm{TaF}_{6}$ : New member located on the modified generalized phase-diagram. J. Phys. Soc. Jpn. 2009, 78, 104717:1-104717:7.

108. Krauzman, M.; Poulet, H.; Pick, R.M. Resonant Raman scattering in a bis-tetramethyltetraselenafulvalene-hexafluorophosphate $\quad(\mathrm{TMTSF})_{2} \mathrm{PF}_{6} \quad$ single crystal. Phys. Rev. B 1986, 33, 99-105.

109. Lüer, L.; Manzoni, C.; Cerullo, G.; Lanzani, G.; Meneghetti, M. Ultrafast dynamics of a charge-transfer dimer as a model for the photoinduced phase transition of charge-transfer compounds. Phys. Rev. Lett. 2007, 99, 027401:1-027401:4.

110. We should note that also for (TMTTF) ${ }_{2} \mathrm{PF}_{6}$ some feature occurs around $75 \mathrm{~cm}^{-1}$ that becomes more obvious in the reflectivity ratio. However, it might be an artefact due to the vicinity of the strong modes at 68 and $84 \mathrm{~cm}^{-1}$.

111. Meneghetti et al. [59] report medium weak modes at 75 and $110 \mathrm{~cm}^{-1}$ in (TMTTF) ${ }_{2} \mathrm{Br}$ and (TMTTF $)_{2} \mathrm{ClO}_{4}$, but do not assign them. They calculate the $\nu_{54}\left(b_{2 u}\right)$ to be located at $75 \mathrm{~cm}^{-1}$.

112. Eldridge, J.E.; Homes, C.C.; Bates, F.E.; Bates, G.S. Far-infrared powder absorption measurements of some tetramethyltetraselenafulvalene salts [(TMTSF) $\left.)_{2} \mathrm{X}\right]$. Phys. Rev. B 1985, $32,5156-5162$.

113. Adamo, C.; Barone, V. Toward reliable density functional methods without adjustable parameters: The PBE0 model. J. Chem. Phys. 1999, 110, 6158:1-6158:13.

114. Pouget, J.-P. Bond and charge ordering in low-dimensional organic conductors. Physica B 2012, 407, 1762-1770.

115. bwGRiD (http://www.bw-grid.de), member of the German D-Grid initiative, funded by the Ministry for Education and Research (Bundesministerium fuer Bildung und Forschung) and the Ministry for Science, Research and Arts Baden-Württemberg (Ministerium für Wissenschaft, Forschung und Kunst Baden-Württemberg).

116. Schmidt, M.W.; Baldridge, K.K.; Boatz, J.A.; Elbert, S.T.; Gordon, M.S.; Jensen, J.H.; Matsunaga, N.; Nguyen, K.A.; Su, S.; Windus, T.L.; et al. General atomic and molecular electronic structure system. Comput. Chem. 1993, 14, 1347-1363.

117. Gordon, M.S.; Schmidt, M.W. Advances in Electronic Strcuture Theory: GAMESS a Decade Later. In Theory and Applications of Computational Chemistry: The First Forty Years; Dykstra, C.E., Frenking, G., Kim, K.S., Scuseria, G.E., Eds.; Elsevier: Amsterdam, The Netherlands, 2005; pp. 1167-1189.

118. Carbonniere, P.; Lucca, T.; Pouchan, C.; Rega, N.; Barone, V. Vibrational computations beyond the harmonic approximation: Performances of the B3LYP density functional for semirigid molecules. J. Comput. Chem. 2005, 26, 384-388.

119. Łapiǹsk, A.; Ouahab, L.; Imakubo, T. Spectroscopic and theoretical study on organic donors: DIET, DIEDO and DIETS. Vib. Spectr. 2010, 52, 22-30.

120. Łapiǹsk, A.; Kotov, A.I. Optical properties of the conducting salt (DOEO) ${ }_{4} \mathrm{HgBr}_{4}-$ TCE. Chem. Phys. 2006, 326, 551-562. 
121. Demiralp, A.; Dasgupta, S.; Goddard, W.A, III. Electron-Transfer Boat-Vibration Mechanism for Superconductivity in Organic Molecules Based on BEDT-TTF. J. Am. Chem. Soc. 1995, 117, 8154-8158.

122. Andersson, M.P.; Uvdal, P. New scale factors for harmonic vibrational frequencies using the B3LYP density functional method with the triple- $\zeta$ Basis Set $6-311+\mathrm{G}(\mathrm{d}, \mathrm{p})$. J. Phys. Chem. A 2005, 109, 2937-2941.

(C) 2012 by the authors; licensee MDPI, Basel, Switzerland. This article is an open access article distributed under the terms and conditions of the Creative Commons Attribution license (http://creativecommons.org/licenses/by/3.0/.) 